\title{
Testing for rational bubbles in a co-explosive vector autoregression
}

\author{
Tom Engsted ${ }^{1} \&$ Bent Nielsen ${ }^{2}$
}

24 June 2010

Summary: We derive the parameter restrictions that a standard equity market model implies for a bivariate vector autoregression for stock prices and dividends, and we show how to test these restrictions using likelihood ratio tests. The restrictions, which imply that stock returns are unpredictable, are derived both for a model without bubbles and for a model with a rational bubble. In both cases we show how the restrictions can be tested through standard chi-squared inference. The analysis for the no-bubble case is done within the traditional Johansen model for I(1) variables, while the bubble model is analysed using a co-explosive framework. The methodology is illustrated using US stock prices and dividends for the period 1872-2000.

Keywords: Rational bubbles, Explosiveness and co-explosiveness, Cointegration, Vector autoregression, Likelihood ratio tests.

\section{Introduction}

During the 1980s rational speculative asset bubbles were a hot topic in both theoretical and empirical asset pricing. Tirole $(1982,1985)$ and Diba and Grossman (1988a), among others, investigated the conditions under which bubbles could occur as an equilibrium phenomenon under rational expectations and informationally efficient capital markets, and Flood and Garber (1980), West (1987), Diba and Grossman (1988b), and Froot and Obstfeld (1991) developed econometric testing procedures for rational bubbles. During the 1990s the research agenda for bubbles was broadened to include irrational motives for the occurance of bubbles. Here, the main focus was on how investor overoptimism and herding behaviour may generate - through various amplifying feed-back mechanisms - long-lasting and irrational deviations between market prices and fundamental values (e.g. Shiller, 2000, and Shleifer, 2000), or on how rational and irrational agents together may generate long-lasting bubbles in models with limits to arbitrage (e.g. Abreu and Brunnermeier, 2003).

\footnotetext{
${ }^{1}$ CREATES, School of Economics and Management, Aarhus University, DK-8000 Aarhus C. Email: tengsted@creates.au.dk. CREATES is funded by the Danish National Research Foundation.

${ }^{2}$ Nuffield College, Oxford OX1 1NF, UK. E-mail: bent.nielsen@nuffield.ox.ac.uk. Financial support from ESRC grant RES-000-27-0179 is gratefully acknowledged.
} 
The increased recent focus in the literature on irrational and behavioral motives for bubbles has not diminished the professions interest in rational bubbles. Some researchers continue to consider rational bubbles more plausible than irrationally generated bubbles (e.g. Leroy, 2004), and the dramatic stock price increases during the 1990s have led to a revival of econometric analyses of rational bubbles, see e.g. Bohl (2003), Engsted (2006), and Balke and Wohar (2009). The characterizing feature of a rational bubble is that it is explosive, i.e. it generates an explosive root in the autoregressive representation for prices. Diba and Grossman (1988b) used Bhargava's (1986) von Neumann-like statistic to test the null hypothesis of a unit root against the explosive alternative. They also tested for cointegration between prices and fundamentals (dividends), arguing that in a constant discount factor present value model cointegration precludes bubbles while no cointegration would be consistent with the presence of a rational bubble. Diba and Grossman's methodology has been one of the most cited and applied methodologies in the empirical bubble literature, and the appealing feature of the approach is that it explicitly addresses the explosive nature of a rational bubble. However, the approach has a number of important limitations, as emphasized by Engsted (2006). In particular, by using Bhargava's (1986) test for explosiveness the variable under consideration needs to be at most a first-order autoregressive process, and in carrying out this test the discount factor cannot be estimated but is assumed to be known in advance.

In the present paper we suggest an econometric procedure for analyzing rational bubbles that overcomes the limitations of Diba and Grossman's procedure, and at the same time contains statistical tests of restrictions directly implied by the presence of a rational bubble. Our procedure builds on the co-explosive framework developed by Nielsen (2010) and originally designed for hyper-inflation data, see Nielsen (2008a). It is similar in spirit to the work of Campbell and Shiller (1987, 1988a) and Johansen and Swensen (1999, 2004), but with some important differences. Campbell and Shiller investigate the present value model for stock prices without bubbles. Similar to the analysis in Diba and Grossman (1988b), a central variable in Campbell and Shiller's analysis is the "spread", $S_{t}=P_{t}-D_{t} / R$, between prices $P_{t}$ and dividends $D_{t}$ normalized by a constant expected return $R$. They show that if prices and dividends satisfy a vector autoregression integrated of order one, then $S_{t}$ will be a cointegrating relation and the short term dynamics will satisfy certain restrictions. They propose a two-step procedure in which $S_{t}$ is first constructed through a single-equation cointegration regression. In a second step additional restrictions implied by the model are imposed on the short term dynamics of a vector autoregression for the "spread" $S_{t}$ and the dividend growth $\Delta_{1} D_{t}=D_{t}-D_{t-1}$. As shown by Johansen and Swensen $(1999,2004)$ then, given $S_{t}$, the latter restrictions can also be imposed on a vector autoregression for the observables $P_{t}$ and $D_{t}$.

We review how the restrictions in terms of cointegration and short term dynamics 
can be imposed jointly on a cointegrated vector autoregression for $P_{t}, D_{t}$. A key feature of these restrictions is that excess returns follow a martingale difference sequence. In this context the unknown constant expected return parameter, $R$, is just-identified. If the focus is on the estimation of $R$ in the no-bubble model, only little is gained by the joint estimation approach compared to the two-step procedure. The advantages of the joint approach do, however, become apparent in the bubble case.

In the bubble situation the vector autoregression of prices $P_{t}$ and dividends $D_{t}$ involves both a unit root and an explosive root as alluded to by Diba and Grossman (1988b). The "spread" $S_{t}$ is then a cointegrating relation in the sense of not having a unit root while being explosive, so it is not immediately clear how to estimate the "spread" $S_{t}$ in a first step of a two-step approach. Instead, the joint restrictions relating to the cointegrating relation $S_{t}$, the short term dynamics, as well as non-explosiveness of dividends $D_{t}$, can be viewed as joint restrictions on the vector autoregression for $P_{t}, D_{t}$. These restrictions can be analysed in the context of the co-explosive model suggested by Nielsen (2010). We show how the restrictions can be tested through standard $\chi^{2}$ inference. It is worth noting that in the bubble model the return parameter $R$ is actually over-identified.

We illustrate the methodology using annual US stock prices and dividends for the period 1872-2000. We analyse both the full period and a smaller sub-period, 1974-2000. We find clear evidence of prices being explosive while dividends are nonexplosive. We also find a common unit root between prices and dividends (i.e. "cointegration"). The over-identifying restrictions implied by the bubble model are rejected at standard significance levels in the full sample but not in the sub-sample. Hence, our empirical analysis provides some support for the rational bubble hypothesis.

The rest of the paper is organized as follows. In $\S 2$ we describe the standard equity market model for stock price determination and explain what a rational bubble implies for prices in this model. We derive the restrictions that the no-bubble model implies for the standard I(1) cointegrated vector autoregression, and we compare these restrictions with the restrictions derived by Johansen and Swensen $(1999,2004)$. Next, we derive the testable restrictions of a rational bubble on a bivariate co-explosive vector autoregression for prices and dividends. $\S 3$ contains the likelihood analyses of the models with the various tests on the cointegrating and co-explosive vectors, their associated factor loadings, and the remaining short term parameters. §4 contains the asymptotic analysis with limiting distributions of parameter estimates and test statistics. $\S 5$ reports the empirical results of using the co-explosive framework to analyse US stock prices and dividends. In $\S 6$ we briefly compare our testing procedure with earlier bubbles tests. Finally, $\S 7$ concludes while proofs are given in an appendix. 


\section{Stock price determination with and without bubbles}

The standard stock price determination model is presented along with the well-known bubble solution. As this model does not specify the behaviour for dividends it is only a partial model so a joint model for prices and dividends is needed for empirical analysis. It is reviewed how the model without bubbles can be embedded in a cointegrated vector autoregression. This leads on to showing how the model with bubbles can be embedded in a co-explosive vector autoregression.

\subsection{Standard model for stock price determination}

The standard model for stock price determination is

$$
P_{t}=\frac{1}{1+R} \mathcal{E}_{t}\left(P_{t+1}+D_{t+1}\right)
$$

where $P_{t}$ and $D_{t}$ are real stock prices and dividends, respectively, and $R$ is the expected (required) one-period return on the stock which is assumed to be constant and positive, i.e. $R>0$. The conditional expectations operator $\mathcal{E}_{t}$ is taken with respect to the information set at time $t$, which contains current and past prices and dividends, i.e. $P_{s}, D_{s}$ for $s \leq t$, but the exact dependence is left unspecified for the moment.

The stock price determination model has some implications for the variable $S_{t} \equiv$ $P_{t}-D_{t} / R$, which is called a "spread" by Campbell and Shiller $(1987,1988 \mathrm{a})$. Subtracting $P_{t}$ from equation (2.1), and multiplying by $1+R$, gives the equation

$$
\mathcal{E}_{t-1} M_{t}=0 \quad \text { where } \quad M_{t}=P_{t}+D_{t}-(1+R) P_{t-1} .
$$

In other words, $M_{t}$ is a martingale difference. Defining the one-period stock return as $R_{t}=\left(P_{t}+D_{t}-P_{t-1}\right) / P_{t-1}$, then $(2.2)$ implies that $P_{t-1}^{-1} \mathcal{E}_{t-1} M_{t}=\mathcal{E}_{t-1} R_{t}-R=0$. Thus, the economic interpretation of (2.2) is that the one-period stock return in excess of a constant mean is a martingale difference and thereby unpredictable given lagged information, i.e. the classical version of The Efficient Markets Hypothesis, c.f. Leroy (1989). This martingale difference can be expressed in terms of the "spread" variable by adding and subtracting $R P_{t}$, and writing price growth as $\Delta_{1} P_{t} \equiv P_{t}-P_{t-1}$, to get

$$
M_{t}=(1+R) \Delta_{1} P_{t}-R S_{t} .
$$

Equation (2.3) shows that if, as often assumed, $X_{t}=\left(P_{t}, D_{t}\right)$ is an I(1) variable then $S_{t}$ must be a cointegrating relation and related to $\Delta_{1} P_{t}$ in a specific way.

This cointegrating relationship can also be formulated as a link between dividend growth $\Delta_{1} D_{t}$ and the spread. To see this add and subtract $\{(1+R) / R\} \Delta_{1} D_{t}$ to get

$$
M_{t}=\Delta_{1+R} S_{t}+\left(1+R^{-1}\right) \Delta_{1} D_{t},
$$


where $\Delta_{1+R} S_{t} \equiv S_{t}-(1+R) S_{t-1}$. Equation (2.4) shows that if $P_{t}$ is explosive, as in a rational bubble, while $D_{t}$ remains I $(1)$, then $S_{t}$ will be explosive but without a unit root. The explosiveness is eliminated by the operator $\Delta_{1+R}$.

As indicated, the stock price model is a partial model. In order to make interesting statements and to make inference, some further assumptions to the stochastic behaviour of $X_{t}$ are needed. In effect the partial model is completed by making an assumption to the behaviour of dividends. At first it is useful to discuss the present value formulation of the model, which is common in the literature.

\subsection{Present value formulation}

The stock price determination model is often stated by writing prices as a present value of expected future dividends. Specifically, the model (2.1) has solution

$$
P_{t}=\sum_{s=1}^{\infty} \frac{\mathcal{E}_{t} D_{t+s}}{(1+R)^{s}}+b B_{t}
$$

for $b \in \mathbb{R}$ and where $B_{t}$ obeys (c.f. Diba and Grossman, 1988a,b)

$$
B_{t}=\frac{1}{1+R} \mathcal{E}_{t} B_{t+1}
$$

The variable $B_{t}$ is called a rational bubble, the component of stock prices that reflects self-fulfilling rational expectations of future price increases independently of fundamentals $\mathcal{E}_{t} D_{t+s}$. To see that (2.5) and (2.6) solve (2.1) insert these on the right hand side of (2.1). The solution (2.5) requires that the sum $\sum_{s=1}^{\infty}(1+R)^{-s} \mathcal{E}_{t} D_{t+s}$ is well-defined.

The rational bubble $B_{t}$ defined by (2.6) also satisfies

$$
\Delta_{1+R} B_{t+1} \equiv B_{t+1}-(1+R) B_{t}=\xi_{t+1},
$$

where the rational expectations error $\xi_{t+1}=B_{t+1}-\mathcal{E}_{t} B_{t+1}$ satisfies $\mathcal{E}_{t} \xi_{t+1}=0$. Since $1+R>1$, the bubble is explosive so unless $b=0$ it induces explosiveness in $P_{t}$.

The present value formulation implies an interesting behaviour for the "spread" variable $S_{t}=P_{t}-D_{t} / R$. Due to the identity $R^{-1}=\sum_{s=1}^{\infty}(1+R)^{-s}$ then

$$
S_{t}=P_{t}-\frac{1}{R} D_{t}=\sum_{s=1}^{\infty} \frac{\mathcal{E}_{t}\left(D_{t+s}-D_{t}\right)}{(1+R)^{s}}+b B_{t}
$$

Noting that $D_{t+s}-D_{t}=\sum_{r=1}^{s} \Delta_{1} D_{t+r}$ with $\Delta_{1} D_{t+r} \equiv D_{t+r}-D_{t+r-1}$, and that $(1+R)^{r} \sum_{s=r}^{\infty}(1+R)^{-s}=(1+R) / R$, this in turn implies

$$
S_{t}=P_{t}-\frac{1}{R} D_{t}=\frac{1+R}{R} \sum_{r=1}^{\infty} \frac{\mathcal{E}_{t}\left(\Delta_{1} D_{t+r}\right)}{(1+R)^{r}}+b B_{t} .
$$


This equation shows that $S_{t}$ enherits its stochastic behaviour from $\Delta_{1} D_{t}$ and $B_{t}$.

In the following we will make some additional assumptions to the stochastic behaviour of $X_{t}$ and study their implications. In a first step $X_{t}$ is assumed vector autoregressive in which case the model (2.1) implies certain linear restrictions on the vector autoregressive coefficients. The solutions with and without bubbles emerge if some additional assumptions are imposed. The first case reviews Campbell and Shiller $(1987,1988 \mathrm{a})$ and Johansen and Swensen $(1999,2004)$ where the bubble is absent, $b=0$. Here $X_{t}$ is assumed to have a unit root so $\Delta_{1} D_{t}, S_{t}$ and, hence, $\Delta_{1} P_{t}$ are stationary and linked as in (2.3). In the second case a bubble is present so $b \neq 0$. Here $X_{t}$ is assumed to have a unit root and an explosive root so $\Delta_{1} D_{t}$ and $\Delta_{1+R} S_{t}$ are stationary and linked through (2.4).

\subsection{A vector autoregressive framework}

The model (2.1) has some interesting implications if $X_{t}=\left(P_{t}, D_{t}\right)$ is assumed vector autoregressive. This assumption is common in the literature, see e.g. Campbell and Shiller (1988a) and Johansen and Swensen (1999, 2004), and it is testable. For the moment no assumptions are made to the location of the characteristic roots. A vector autoregression of order $k$ has the format

$$
X_{t}=\sum_{j=1}^{k} A_{j} X_{t-j}+\mu+\varepsilon_{t},
$$

where $A_{j} \in \mathbb{R}^{2 \times 2}$ and $\mu \in \mathbb{R}^{2}$. The errors, $\varepsilon_{t}$, are independent $\mathrm{N}_{2}(0, \Omega)$-distributed, or more generally a martingale difference sequence.

To see the implications of (2.1) for the vector autoregression (2.8), it is convenient to write (2.8) in companion form

$$
\mathbf{X}_{t}=\mathbf{A X}_{t-1}+\boldsymbol{\mu}+\mathbf{e}_{t}
$$

where

$$
\mathbf{A}=\left(\begin{array}{c|c}
A_{1}, \ldots A_{k-1} & A_{k} \\
\hline I_{2 k-2} & 0
\end{array}\right), \quad \mathbf{X}_{t-1}=\left(\begin{array}{c}
X_{t-1} \\
\vdots \\
X_{t-k}
\end{array}\right), \quad \boldsymbol{\mu}=\left(\begin{array}{c}
\mu \\
0
\end{array}\right), \quad \mathbf{e}_{t}=\left(\begin{array}{c}
\varepsilon_{t} \\
0
\end{array}\right)
$$

Equation (2.2) implies that the companion vector $\mathbf{X}_{t}$ satisfies

$$
\nu^{\prime} \mathcal{E}_{t-1} \mathbf{X}_{t}=0 \quad \text { where } \quad \nu^{\prime}=\{1,1,-(1+R), 0, \ldots, 0\} .
$$

As the innovations satisfy $\mathcal{E}_{t-1} \mathbf{e}_{t}=0$, it must hold that $\nu^{\prime} \mathbf{A}=0$, which is equivalent to the constraints, for $j=2, \ldots, k$,

$$
(1,1) A_{1}=(1+R, 0), \quad(1,1) A_{j}=0, \quad(1,1) \mu=0 .
$$


Thus, (2.1) together with the vector autoregressive assumption implies the constraints (2.9). Some further assumptions to the locations of the characteristic roots of the vector autoregression are needed for two reasons: first, to be able to distinguish between the cases with and without bubbles, and, secondly, to be able to conduct reliable inference about the constraints (2.9).

\subsection{The case without a rational bubble}

We now analyse the standard case without a rational bubble. Campbell and Shiller (1987, 1988a) discuss this case under the additional assumption that the vector autoregression has one unit root in such a way that the spread, $S_{t}=P_{t}-D_{t} / R$, and dividend growth, $\Delta_{1} D_{t}$, are stationary. Campbell and Shiller estimate the structural parameter $R$ super-consistently in a first-step cointegration regression, and this parameter is subsequently treated as known in a vector autoregressive analysis for $\Delta_{1} D_{t}$ are $S_{t}$. They derive the restrictions that (2.1) implies for this particular vector autoregression. Johansen and Swensen $(1999,2004)$ instead work with a vector autoregression for $X_{t}=\left(P_{t}, D_{t}\right)^{\prime}$ and show how the restrictions implied by (2.1) can be tested in an I(1) framework. In their theoretical analysis they assume that the cointegrating vector $S_{t}$ is known; however, in their empirical illustrations they discuss the case of unknown cointegration vector. We discuss the latter case in further detail.

It is convenient to reparametrise the vector autoregression in error correction form:

$$
\Delta_{1} X_{t}=\Pi X_{t-1}+\sum_{j=1}^{k-1} \Gamma_{j} \Delta_{1} X_{t-j}+\mu+\varepsilon_{t},
$$

where $\Pi, \Gamma_{j} \in \mathbb{R}^{2 \times 2}$ and $\mu \in \mathbb{R}^{2}$. This is equivalent to the vector autoregression (2.8) with $\Pi=\sum_{j=1}^{k} A_{j}-I_{2}$ and $\Gamma_{j}=-\sum_{\ell=j+1}^{k} A_{\ell}$, see Johansen $(1995, \S 4.1)$. The restrictions to the $A_{j}$-coefficients in (2.9) are then equivalent to, for $j=1, \ldots, k-1$,

$$
(1,1) \Pi=R(1,-1 / R), \quad(1,1) \Gamma_{j}=0, \quad(1,1) \mu=0 .
$$

These are the restrictions discussed in the theory part of Johansen and Swensen (1999, 2004) under the assumption that $X_{t} \sim \mathrm{I}(1)$ and reduced rank of $\Pi$.

With the assumption that $X_{t}$ is I(1) with one unit root and the remaining roots stationary, $\Pi$ has a rank of unity so $\Pi=\alpha \beta^{\prime}$ where $\beta \in \mathbb{R}^{2 \times 1}$ is the cointegrating vector and $\alpha \in \mathbb{R}^{2 \times 1}$ is the associated vector of factor loadings. The restriction $(1,1) \Pi=R(1,-1 / R)$ in $(2.11)$ then implies that

$$
\beta=(1,-1 / R)^{\prime} .
$$

The last argument also shows that $(1,1) \alpha=R$. It is interesting to note that these arguments do not exploit the multiplicity of the unit root so they would also apply 
to I(2) situations and explosive situations as will be seen in $§ 2.5$. Note also that the restriction in (2.12) identifies the parameter $R$ as pointed out in connection with the illustration of Johansen and Swensen (2004).

Another representation of the process is the Granger-Johansen representation: With the assumption that $X_{t}$ is I(1) with one unit root, and the remaining roots stationary, the process can be interpreted through its Granger-Johansen representation, see Johansen (1995, Theorem 4.1). This shows that $\beta^{\prime} X_{t}$ and $\Delta_{1} X_{t}$ can be given a stationary distribution while

$$
X_{t}=C \sum_{s=1}^{t} \varepsilon_{s}+Y_{t}+\tau_{c}+\tau_{l} t
$$

where $C=\beta_{\perp}\left(\alpha_{\perp}^{\prime} \Psi \beta_{\perp}\right)^{-1} \alpha_{\perp}^{\prime}$ for $\Psi=I_{2}-\sum_{j=1}^{k-1} \Gamma$ while $Y_{t}$ is a stationary process. The deterministic components are $\tau_{l}=C \mu$ while $\tau_{c}$ depends on parameters and the initial observations. The linear trend is avoided by assuming $\mu=\alpha \delta$ for some $\delta \in \mathbb{R}$ so $\tau_{l}=C \mu=0$. The restriction $(1,1) \mu=0$ in (2.11) along with the finding that $(1,1) \alpha=R$ then implies that $\delta=0$.

We now have two sets of restrictions: those in (2.11) arising from the vector autoregressive assumption, and the restrictions to $\beta$ and $\mu$ arising from the unit root assumption. These restrictions imply

$$
(1,1) \alpha=R, \quad \beta=(1,-1 / R)^{\prime}, \quad(1,1) \Gamma_{j}=0, \quad(1,1) \mu=0,
$$

in which $R$ appears twice, or, equivalently,

$$
(1,1) \Pi(0,1)^{\prime}=-1, \quad \beta=(1,-1 / R)^{\prime}, \quad(1,1) \Gamma_{j}=0, \quad(1,1) \mu=0 .
$$

With these restrictions the model (2.10) can be rewritten in terms of an equation for the martingale difference $M_{t}$ in (2.2) and an equation for dividend growth. These equations are obtained by taking the linear combinations $(1,1) \Delta_{1} X_{t}$ and $(0,1) \Delta_{1} X_{t}$ of equation (2.10). They are given by

$$
\begin{aligned}
M_{t} & =\varepsilon_{M, t}, \\
\Delta_{1} D_{t} & =\alpha_{D} S_{t-1}+\sum_{j=1}^{k-1} \Gamma_{j, D} \Delta_{1} X_{t-j}+\mu_{D}+\varepsilon_{D, t},
\end{aligned}
$$

where $\left(\alpha_{D}, \Gamma_{j, D}, \mu_{D}\right)=(0,1)\left(\alpha, \Gamma_{j}, \mu\right)$ while $\varepsilon_{M, t}=(1,1) \varepsilon_{t}$ and $\varepsilon_{D, t}=(0,1) \varepsilon_{t}$. These equations show that in the context of a vector autoregression for $X_{t}$, which is $\mathrm{I}(1)$, then the stock price model implies that $M_{t}$ is a martingale difference along with an equation for dividend growth in terms of the lagged "spread" and lagged growth of dividends and prices. In other words, the equation for dividends show how the partial model (2.1) is completed in the context of an I(1) vector autoregression. Johansen and Swensen (2004) discuss equations for $(1+R)^{-1} M_{t}$ and $\Delta_{1}\left(P_{t}-D_{t}\right)$ which will give an equivalent representation of the model. 


\subsubsection{Weak exogeneity}

We close this section with a brief discussion of weak exogeneity. A feature of equation (2.16) is that there is feedback from the lagged "spread", $S_{t-1}$, to dividend growth, $\Delta_{1} D_{t}$. For a first order model, $k=1$, the only feedback is through the coefficient $\alpha_{D}$ which has to be positive under the I(1) assumption. In other words, dividends cannot be weakly exogenous for the "spread". In higher-order models there are two sources of feedback: directly from $S_{t-1}$ with coefficient $\alpha_{D}$ as well as through lagged price growth with coefficients $\Gamma_{j, D}(1,0)^{\prime}$. In this situation the I(1) assumption is consistent with the coefficient $\alpha_{D}$ being positive or negative or indeed zero.

For a first order model, $k=1$, the argument is as follows. The Granger-Johansen representation (Johansen 1995, Theorem 4.2) gives a condition for stationarity of $S_{t}=\beta^{\prime} X_{t}$ which is that $\left|1+\beta^{\prime} \alpha\right|<1$. Due to the restriction $(1,1) \alpha=R$ we can write $\alpha=(\lambda, R-\lambda)^{\prime}$ for some parameter $\lambda$. Since $\beta=(1,-1 / R)$ in $(2.13)$ then $1+\beta^{\prime} \alpha=1+\lambda-(1-\lambda / R)$. This is bounded by one in absolute value when $|\lambda|<$ $R /(1+R)$. In particular, the coefficient $\alpha_{D}=R-\lambda$ must be in the positive range from $R^{2} /(1+R)>0$ to $R+R /(1+R)$. In particular, the possibility that $\alpha_{D}=0$, implying that dividends form a pure random walk, is then ruled out.

For a second order model, $k=2$, it is complicated to analyse the characteristic polynomial in general. An example, that obeys (2.2) and where $\alpha_{D}=0$, is

$$
\Delta_{1}\left(\begin{array}{c}
P_{t} \\
D_{t}
\end{array}\right)=\left(\begin{array}{c}
R \\
0
\end{array}\right) S_{t-1}+\left(\begin{array}{c}
-2 R \\
2 R
\end{array}\right) \Delta_{1} P_{t-1}+\varepsilon_{t},
$$

for which the characteristic polynomial is $(1-z)\{1-(1-R) z\}$ giving a unit root and a stationary root of $1 /(1-R)>1$. In this example the apparent explosive reaction in prices to $S_{t-1}$, and the lack of reaction in dividends to $S_{t-1}$, are compensated for by the lagged price growth. This example is akin to the example in Johansen (1995, Exercise 4.3). It shows that for $k>1$, one should be careful in interpreting the signs and magnitudes of the individual factor loadings in $\alpha$. All one can say is that under the stock price model $(2.1)$, the restriction $(1,1) \alpha=R$ has to hold, see (2.13).

In summary, in the context of the cointegrated vector autoregression (2.10), the stock price model (2.1) without a bubble implies the parameter restrictions in (2.13). These restrictions imply that $M_{t}$, as defined in (2.2), is a martingale difference meaning that stock returns are unpredictable. In $\S 3$ we explain how to test these restrictions within a likelihood framework.

\subsection{The case with a rational bubble}

The case with a rational bubble can be analysed much the same way as the case without a bubble. In this case the additional assumption is made that the vector autoregression has one unit root, and one explosive root $\rho>1$. 
Before exploiting the additional assumptions to the characteristic roots it is convenient to reparametrise the vector autoregression in error correction form aimed at co-explosive behaviour, c.f. Nielsen (2010):

$$
\Delta_{1} \Delta_{\rho} X_{t}=\Pi_{1} \Delta_{\rho} X_{t-1}+\Pi_{\rho} \Delta_{1} X_{t-1}+\sum_{j=1}^{k-2} \Phi_{j} \Delta_{1} \Delta_{\rho} X_{t-j}+\mu+\varepsilon_{t}
$$

where $\Delta_{\rho} X_{t} \equiv X_{t}-\rho X_{t-1}$, and where $\Pi_{1}, \Pi_{\rho}, \Phi_{j} \in \mathbb{R}^{2 \times 2}$ and $\rho \in \mathbb{R}$. This is equivalent to the vector autoregression (2.8) with

$$
\Pi_{1}=\frac{\Pi}{1-\rho}, \quad \Pi_{\rho}=-\rho\left(I_{p}+\Pi_{1}-\sum_{j=1}^{k-1} \rho^{-j} \Gamma_{j}\right), \quad \Phi_{j}=\sum_{l=j+1}^{k-1} \rho^{j-l} \Gamma_{l},
$$

see Nielsen $(2010, \S 2.3)$. The restrictions to the $\Pi, \Gamma_{j}$, and $\mu$ coefficients in $(2.11)$ are then equivalent to, for $j=1, \ldots, k-2$,

$$
\begin{aligned}
(1,1) \Pi_{1} & =\frac{R}{1-\rho}(1,-1 / R), \quad(1,1) \Phi_{j}=0, \quad(1,1) \mu=0, \\
(1,1) \Pi_{\rho} & =\frac{-\rho}{1-\rho}(R,-1)-\rho(1,1)=\frac{-\rho}{1-\rho}(R+1-\rho,-\rho) .
\end{aligned}
$$

The additional assumptions that $X_{t}$ has one unit root and one explosive root and that the remaining roots are stationary are accommodated by reduced rank restrictions so $\Pi_{1}=\alpha_{1} \beta_{1}^{\prime}$ and $\Pi_{\rho}=\alpha_{\rho} \beta_{\rho}^{\prime}$ where $\alpha_{1}, \beta_{1}, \alpha_{\rho}, \beta_{\rho} \in \mathbb{R}^{2}$. The process can be interpreted through its Granger-Johansen representation. Such a representation was given in Nielsen (2010, Theorem 1) and a more detailed version is given as Theorem A.1 in the Appendix. This shows that $\beta_{1}^{\prime} \Delta_{\rho} X_{t}, \beta_{\rho}^{\prime} \Delta_{1} X_{t}$ and $\Delta_{1} \Delta_{\rho} X_{t}$ can be given a stationary distribution while

$$
X_{t}=\frac{1}{1-\rho} C_{1}\left(A_{1}+\sum_{s=1}^{t} \varepsilon_{s}\right)+\frac{1}{\rho-1} C_{\rho} \rho^{t}\left(A_{\rho}+\sum_{s=1}^{t} \rho^{-s} \varepsilon_{s}\right)+Y_{t}+\tau .
$$

Here $Y_{t}$ is some stationary process, $A_{1}, A_{\rho}$ depend on parameters and initial values. The impact matrices satisfy $\beta_{1}^{\prime} C_{1}=0$ so $\beta_{1}^{\prime} X_{t}$ has no random walk component while $\beta_{\rho}^{\prime} C_{\rho}=0$ so $\beta_{\rho}^{\prime} X_{t}$ has no explosive component. Thus, $\beta_{1}$ are cointegrating vectors and $\beta_{\rho}$ are co-explosive vectors. The explosive common trend $\rho^{t}\left(A_{\rho}+\sum_{s=1}^{t} \rho^{-s} \varepsilon_{s}\right)$ then represents the bubble $B_{t}$ in (2.6). Detailed definitions of these coefficients are given in Theorem A.1 in the appendix.

With the above assumptions, three additional restrictions emerge and the restrictions (2.19)-(2.20) simplify. In order to match the explosive common trend and the bubble, it must hold that $\rho=1+R$, see (2.6). The additional restrictions can be 
deduced in two ways. First, $\Delta_{\rho} X_{t}$ and $\Delta_{\rho} S_{t}$ must be non-explosive while $\Delta_{1} D_{t}$ has no unit root. Equation (2.4) then implies that $\Delta_{\rho} S_{t}$ and $\Delta_{1} D_{t}$ must both be stationary. Thus, $S_{t}$ is the cointegrating relation and $D_{t}$ is the co-explosive relation so that

$$
\beta_{1}=(1,-1 / R)^{\prime}, \quad \beta_{\rho}=(0,1)^{\prime} .
$$

Secondly, following Nielsen (2010), the assumption to the roots implies that $\Pi_{1}, \Pi_{\rho}$ both have rank of unity so $\Pi_{1}=\alpha_{1} \beta_{1}^{\prime}$ and $\Pi_{\rho}=\alpha_{\rho} \beta_{\rho}^{\prime}$ where $\alpha_{1}, \beta_{1}, \alpha_{\rho}, \beta_{\rho} \in \mathbb{R}^{2 \times 1}$. Since the explosive root is $\rho=1+R$, the restrictions (2.19)-(2.20) simplify as

$$
(1,1) \Pi_{1}=(-1,1 / R), \quad(1,1) \Pi_{\rho}=\left\{0,-(1+R)^{2} / R\right\}, \quad(1,1) \Phi_{j}=0, \quad(1,1) \mu=0 .
$$

This in turn implies the expressions for $\beta_{1}$ and $\beta_{\rho}$ in (2.21). The latter argument also shows that $(1,1) \alpha_{1}=-1$ and $(1,1) \alpha_{\rho}=-(1+R)^{2} / R$.

We now have two sets of restrictions: those in (2.19)-(2.20) arising from the vector autoregressive assumption as well as the restriction to $\rho, \beta_{1}$ and $\beta_{\rho}$ arising from the assumptions to the characteristic roots. These restrictions together imply

$$
\begin{aligned}
& (1,1) \alpha_{1}=-1, \quad \beta_{1}=(1,-1 / R)^{\prime}, \quad \rho=1+R, \quad(1,1) \mu=0, \\
& (1,1) \alpha_{\rho}=-(1+R)^{2} / R, \quad \beta_{\rho}=(0,1)^{\prime}, \quad(1,1) \Phi_{j}=0 .
\end{aligned}
$$

With these restrictions the model (2.17) can be rewritten in terms of an equation for the martingale difference $M_{t}$ in (2.2) and an equation for dividend growth. These equations arise by taking the linear combinations $(1,1) \Delta_{1} \Delta_{\rho} X_{t}$ and $(0,1) \Delta_{1} \Delta_{\rho} X_{t}$ of equation (2.17). They are given by

$$
\begin{aligned}
M_{t}= & \varepsilon_{M, t} \\
\Delta_{1} \Delta_{\rho} D_{t}= & \alpha_{1, D} \Delta_{\rho} S_{t-1}+\alpha_{\rho, D} \Delta_{1} D_{t-1} \\
& +\sum_{j=1}^{k-2} \Phi_{j, D} \Delta_{1} \Delta_{\rho} X_{t-j}+\mu_{D}+\varepsilon_{D, t},
\end{aligned}
$$

where $\left(\alpha_{1, D}, \alpha_{\rho, D}, \Phi_{j, D}, \mu_{D}\right)=(0,1)\left(\alpha_{1}, \alpha_{\rho}, \Phi_{j}, \mu\right)$ while $\varepsilon_{M, t}=(1,1) \varepsilon_{t}$ and $\varepsilon_{D, t}=$ $(0,1) \varepsilon_{t}$. These equations show that in the context of a vector autoregression for $X_{t}$, which is I(1) with an explosive root, the stock price model implies that $M_{t}$ is a martingale difference along with an equation for dividends in terms of the lagged "spread" and lagged filtered growth of dividends and prices. As before, the equation for dividends shows how the partial model (2.1) is completed in the context of an explosive vector autoregression.

There are no simple restrictions to the feedback coefficients $\alpha_{1, D}$ and $\alpha_{\rho, D}$ in the dividend equation (2.24). This is because the simplest model has two lags, $k=2$, 
for which the dynamics is rather complicated as we saw for the non-bubble model in $\S 2.4 .1$. As an illustration, parametrise the model (2.17) with restrictions (2.22) as

$$
\Delta_{1} \Delta_{\rho}\left(\begin{array}{c}
P_{t} \\
D_{t}
\end{array}\right)=\left(\begin{array}{c}
\lambda \\
-1-\lambda
\end{array}\right) \Delta_{\rho} S_{t-1}+\left(\begin{array}{c}
\gamma \\
-(1+R)^{2} / R-\gamma
\end{array}\right) \Delta_{1} D_{t-1}+\varepsilon_{t} .
$$

The characteristic polynomial is $(1-z)\{1-(1+R) z\}\{1+(\gamma-\lambda-\lambda / R) z\}$, which has one unit root, one explosive root at $1 /(1+R)$, and one stationary root if $-1<$ $\gamma-\lambda-\lambda / R<1$. It links $\alpha_{1, D}$ and $\alpha_{\rho, D}$ in a complicated way. For instance, it is possible that $\alpha_{1, D}=0$, that is $\lambda=-1$, as long as $-1<\gamma+1+1 / R<1$, that is $\left|\alpha_{\rho, D}+1+R\right|<1$.

In summary, in the context of the co-explosive vector autoregression (2.17), the stock price model (2.1) with a bubble implies the parameter restrictions in (2.22). Just as in the case with no bubble, these restrictions imply that $M_{t}$, as defined in (2.2), is a martingale difference meaning that stock returns are unpredictable. In the next section we explain how to test these restrictions within a likelihood framework.

\section{Likelihood analysis of the models}

In the previous section the stock price model was analysed in the context of a vector autoregression for $X_{t}=\left(P_{t}, D_{t}\right)^{\prime}$. In particular, it was shown which parameter restrictions are implied by a rational bubble. In the following it is shown how these parameter restrictions can be tested through a likelihood analysis.

\subsection{Unrestricted vector autoregression}

The data consists of a bivariate time series $X_{1-k}, \ldots, X_{0}, X_{1}, \ldots, X_{T}$ where $X_{t}=$ $\left(P_{t}, D_{t}\right)^{\prime}$. The unrestricted vector autoregression of $(2.8)$ is of the form

$$
\mathrm{M}: \quad X_{t}=\sum_{j=1}^{k} A_{j} X_{t-j}+\mu+\varepsilon_{t}
$$

conditional on the initial observations $X_{1-k}, \ldots, X_{0}$. The parameters satisfy $A_{j} \in$ $\mathbb{R}^{2 \times 2}, \mu \in \mathbb{R}^{2}$ and $\varepsilon_{t}$ are independently $\mathrm{N}_{2}(0, \Omega)$-distributed. While the normality assumption is important for defining the likelihood it can be replaced with a martingale difference assumptions for most purposes. Indeed, for consistency of the estimators and all but one of the suggested tests, martingale difference assumptions suffice.

From the least squares estimates of the dynamic parameters $A_{j}$ the characteristic roots can be computed. A first indication of explosiveness would be if one of these roots appear in the explosive region. 
To secure that this model is statistically well-specified, the usual diagnostic tests are carried out as far as they are valid in the presence of both a unit root and an explosive root. Nielsen (2006a,b, 2008b) has shown that the usual procedures for laglength determinations are valid. This includes the information criteria approaches of Schwarz (1978) and Hannan and Quinn (1979) and the auxillary regression approaches of Godfrey (1978), Breusch (1978) and Pagan (1984). Engler and Nielsen (2009) show that QQ-plots for comparing the empirical distribution of the residuals with a normal distribution are valid. The properties of tests for autoregressive conditional heteroskedasticity by Engle (1982) and the recursive Chow-type tests of Doornik and Hendry (2001) are currently explored by one of us.

\subsection{Testing the cointegration rank}

The next step in the analysis is to establish the cointegration rank. With that in mind the model is reparametrised in error correction form, as in (2.10), as

$$
\mathrm{M}: \quad \Delta_{1} X_{t}=\Pi X_{t-1}+\sum_{j=1}^{k-1} \Gamma_{j} \Delta_{1} X_{t-j}+\mu+\varepsilon_{t} .
$$

The cointegration rank hypothesis of interest is that

$$
\mathrm{H}_{1}: \quad r=\operatorname{rank}(\Pi, \mu)=1 \quad \text { or } \quad(\Pi, \mu)=\alpha\left(\beta^{\prime}+\delta^{\prime}\right) \quad \text { for } \quad \alpha, \beta \in \mathbb{R}^{2 \times 1}, \delta \in \mathbb{R} .
$$

Under this reduced rank hypothesis, we denote the model $M_{1}$. The constant is restricted to the cointegrating space partly because it is appropriate in the empirical illustration and partly because it simplifies the determination of the rank, see Nielsen and Rahbek (2000). It would be possible to extend the analysis to the case of an unrestricted constant or even a restricted linear trend.

In practice, the rank is estimated through a sequential testing procedure as shown by Johansen $(1995, \S 13)$, see also Nielsen and Rahbek (2000). The rank is estimated to be unity if the hypothesis

$$
\mathrm{H}_{1,0}: \quad \Pi=0, \quad \mu=0,
$$

is rejected and the hypothesis

$$
\mathrm{H}_{1,1}: \quad \operatorname{rank}(\Pi, \mu) \leq 1,
$$

cannot be rejected. By testing the hypotheses in this sequence the asymptotic properties of the rank estimator can be controlled.

Results concerning the likelihood ratio tests for these hypotheses against $M$ are available for both the non-bubble and the bubble case. In the non-bubble case it 
can be assumed that the process is I(1), that is, it has unit roots of multiplicity one along stationary roots. This situation is analysed by Johansen (1995, Theorem 6.2) and the test statistics are found to have Dickey-Fuller type distributions. For the bubble case where the process also has one explosive root, results are available for the univariate case in Nielsen (2001) and for the multivariate case in Nielsen (2010, Theorem 4). Strictly speaking, the latter multivariate result covers the situation with a linear trend component, but the result could presumably be extended to the specification of deterministic components used here.

The further analysis now depends on the presence of an explosive root and hence a bubble. For the non-bubble case the analysis is given in $\S 3.3$, whereas for the bubble case the analysis is given in $\S 3.4$.

\subsection{Testing the model without a bubble}

The theory model without a bubble implies the restricted model given by (2.15)-(2.16). The errors $\varepsilon_{M, t}$ and $\varepsilon_{D, t}$ may be correlated, but by assuming normality and letting $\omega$ denote the population regression coefficient of $\varepsilon_{D, t}=(0,1) \varepsilon_{t}$ on $\varepsilon_{M, t}=(1,1) \varepsilon_{t}$, these equations can be rewritten as

$$
\begin{aligned}
M_{t} & =\varepsilon_{M, t}, \\
\Delta_{1} D_{t} & =\alpha_{D} S_{t-1}+\sum_{j=1}^{k-1} \Gamma_{j, D} \Delta_{1} X_{t-j}+\mu_{D}+\omega M_{t}+\varepsilon_{D \cdot M, t},
\end{aligned}
$$

where the errors $\varepsilon_{M, t}$ and $\varepsilon_{D \cdot M, t}=\varepsilon_{D, t}-\omega \varepsilon_{M, t}$ are uncorrelated. The likelihood implied by these equations is maximised through a profile argument. For a known $R$, the profile likelihood is maximised by estimating these two unrelated regressions. The likelihood is then maximised by maximising over $R$.

The likelihood ratio test statistic for the restrictions (2.14) within the model $M_{1}$ would presumably by $\chi^{2}$ with $1+2(k-1)+1=2 k$ degrees of freedom. In order to prove this formally one would have to combine techniques from two sets of results. The first result concerns a test for the restrictions on $\Pi$ in the model $M_{1}$. Boswijk and Doornik (2004) show that this is $\chi^{2}$. The second result concerns a test for the restrictions on the $\Gamma_{j}$-parameters. Johansen (1995, Theorem 13.5) shows that the unrestricted estimators $\hat{\Gamma}_{j}$ in model $\mathrm{M}_{1}$ are asymptotically normal which would presumably imply that the test on the $\Gamma_{j}$-parameters is also $\chi^{2}$. Johansen and Swensen (2004, Remark 4) discuss the joint test of all the restrictions, albeit with a known value of $R$.

\subsection{Testing the model with a bubble}

We now derive the tests of the restrictions implied by the bubble hypothesis, i.e. (2.22). First, we reparametrise the vector autoregression in co-explosive form and, 
based on that, we then set up null hypotheses on the cointegrating and co-explosive vectors, their factor loadings, and the remaining short term parameters. In the end this leads to a four-step procedure for testing for bubbles.

\subsubsection{Testing that dividends are non-explosive}

Suppose the cointegrating rank has been determined to be unity by the approach in $\S 3.2$ and that estimated parameters imply one explosive root, $\hat{\rho}$, say. The vector autoregressive model can then be rewritten in co-explosive form as discussed in $\S 2.5$ :

$$
\mathrm{M}_{1}: \quad \Delta_{1} \Delta_{\rho} X_{t}=\alpha_{1} \beta_{1}^{\prime} \Delta_{\rho} X_{t-1}+\alpha_{\rho} \beta_{\rho}^{\prime} \Delta_{1} X_{t-1}+\sum_{j=1}^{k-2} \Phi_{j} \Delta_{1} \Delta_{\rho} X_{t-j}+\mu+\varepsilon_{t} .
$$

Estimates for the parameters can be computed from those of a standard cointegrated vector autoregression using the identities (2.18). By construction the estimate $\hat{\alpha}_{\rho} \hat{\beta}_{\rho}^{\prime}$ will have reduced rank of unity, so the estimates $\hat{\alpha}_{\rho}, \hat{\beta}_{\rho} \in \mathbb{R}^{2}$ can be found as the left and right eigenvectors, respectively.

A central assumption in $\S 2.5$ is that dividends are difference stationary without any explosive features. This results in a simple hypothesis on the co-explosive vector

$$
\mathrm{H}_{D}: \quad \beta_{\rho}=(0,1)^{\prime} .
$$

Under the hypothesis $\mathrm{H}_{D}$, the model $\mathrm{M}_{1}$ reduces to

$$
\mathrm{M}_{1 D}: \quad \Delta_{1} \Delta_{\rho} X_{t}=\alpha_{1} \beta_{1}^{\prime} \Delta_{\rho} X_{t-1}+\alpha_{\rho} \Delta_{1} D_{t-1}+\sum_{j=1}^{k-2} \Phi_{j} \Delta_{1} \Delta_{\rho} X_{t-j}+\mu+\varepsilon_{t} .
$$

The likelihood of the model $\mathrm{M}_{1 D}$ is analysed through a profile argument. For a given $\rho$, the likelihood is maximised by a reduced rank regression of $\Delta_{1} \Delta_{\rho} X_{t}$ on $\Delta_{\rho} X_{t-1}$ correcting for $\Delta_{1} D_{t-1}$, lags $\Delta_{1} \Delta_{\rho} X_{t-j}$, and a constant. This in turn can be maximised by a grid search over $\rho$.

The likelihood ratio test statistic for testing $\mathrm{H}_{D}$ in $\mathrm{M}_{1}$ is asymptotically $\chi^{2}(p-1)$ as shown by Nielsen (2010, Corollary 1). In our case the dimension of $X_{t}$ is $p=2$. This result requires that a certain linear combination of the innovations are normally distributed. The linear combination in question is $\tau^{\prime} \varepsilon_{t}$ where $\tau$ is the orthogonal complement of $\Psi_{\rho} \beta_{\rho \perp}$.

\subsubsection{Testing the link between the spread and the explosive root}

Conditional on $\mathrm{H}_{D}$ above, the restriction between the cointegrating vector and the explosive root can be formulated as a "spread" restriction:

$$
\mathrm{H}_{S}: \quad \beta_{1}=(1,-1 / R)^{\prime}, \quad \text { where } R=\rho-1 \text {. }
$$


Under this restriction, the model reduces to

$$
\mathrm{M}_{1 D S}: \quad \Delta_{1} \Delta_{\rho} X_{t}=\alpha_{1} \Delta_{\rho} S_{t-1}+\alpha_{\rho} \Delta_{1} D_{t-1}+\sum_{j=1}^{k-2} \Phi_{j} \Delta_{1} \Delta_{\rho} X_{t-j}+\mu+\varepsilon_{t},
$$

where $S_{t}=P_{t}-\frac{1}{R} D_{t}$ is denoted the "spread". Again, the likelihood is maximized over $\rho$. The likelihood ratio test statistic for testing $\mathrm{H}_{S}$ in $\mathrm{M}_{1 D}$ is asymptotically $\chi^{2}(1)$. We do not show this directly. Rather, in $\S 4$ we show that the test statistic for the joint hypothesis $\mathrm{H}_{S}, \mathrm{H}_{B}$ in $\mathrm{M}_{1 D}$ is a sum of two $\chi^{2}$ statistics of which one corresponds to the test for $\mathrm{H}_{S}$ in $\mathrm{M}_{1 D}$.

\subsubsection{Testing the martingale difference restrictions}

The final set of restrictions in (2.22) implied by the theory model with a bubble is:

$$
\mathrm{H}_{B}: \quad(1,1) \alpha_{1}=-1, \quad(1,1) \alpha_{\rho}=-(1+R)^{2} / R, \quad(1,1) \Phi_{j}=0, \quad(1,1) \mu=0 .
$$

In combination with the restricted constant, $\mu=\alpha_{1} \delta^{\prime}$, the above constraints imply that $\mu=0$. Under these restrictions, the model reduces to the least squares regressions given by (2.23)-(2.24). As in section 3.3 the errors may be correlated, so by assuming normality and letting $\omega$ denote the population regression coefficient of $\varepsilon_{D, t}=(0,1) \varepsilon_{t}$ on $\varepsilon_{M, t}=(1,1) \varepsilon_{t}$, these equations can be rewritten as

$$
\begin{aligned}
M_{1 D S B}: \quad & \varepsilon_{M, t}, \\
\Delta_{1} D_{t}= & \alpha_{1, D} \Delta_{\rho} S_{t-1}+\alpha_{\rho, D} \Delta_{1} D_{t-1} \\
& +\sum_{j=1}^{k-2} \Phi_{j, D} \Delta_{1} \Delta_{\rho} X_{t-j}+\mu_{D}+\omega M_{t}+\varepsilon_{D \cdot M, t},
\end{aligned}
$$

where the errors $\varepsilon_{M, t}$ and $\varepsilon_{D \cdot M, t}=\varepsilon_{D, t}-\omega \varepsilon_{M, t}$ are uncorrelated. We denote this model $\mathrm{M}_{1 D S B}$. The likelihood implied by these equations is maximised through a profile argument using that for a known $R$ the regressions (3.6) and (3.7) are unrelated. The likelihood is then maximised by maximising over $R$.

The likelihood ratio test statistic for the restricted model $\mathrm{M}_{1 D S B}$, i.e. (3.6)-(3.7), within the model $\mathbf{M}_{1 D}$, is shown to be asymptotically $\chi^{2}(2 k)$ in $\S 4$.

\subsection{Summary of procedure for testing the model with a bubble}

To summarize, our suggested procedure for testing for a rational bubble consists of the following four steps:

Step 1: Fit an unrestricted VAR model for $X_{t}=\left(P_{t}, D_{t}\right)^{\prime}$ and check that it is econometrically well-specified using misspecification tests. Compute the characteristic 
roots from the matrix lag polynomial and see if the largest root is larger than 1, i.e. $\rho>1$. Test that the cointegration rank is $r=1$ using Johansen's test. The restricted model is denoted $\mathrm{M}_{1}$.

Step 2: Given a cointegration rank of $r=1$ and that $\rho>1$, test the hypothesis $\mathrm{H}_{D}: \beta_{\rho}=(0,1)^{\prime}$, meaning that $D_{t}$ is non-explosive. The restricted model is denoted $\mathrm{M}_{1 D}$. The test statistic for $\mathrm{H}_{D}$ in $\mathrm{M}_{1}$ is asymptotically $\chi^{2}(1)$.

Step 3: Test the hypothesis $\mathrm{H}_{S}: \beta_{1}=\left(1,-R^{-1}\right)^{\prime}$ with $R=\rho-1$. This restriction relates the expected return in the theory model linearly to the explosive root in the econometric model. The restricted model is denoted $\mathrm{M}_{1 D S}$. The test statistic for $\mathrm{H}_{S}$ in $\mathrm{M}_{1 D}$ is asymptotically $\chi^{2}(1)$.

Step 4: Test the final restrictions implied by the bubble model, $\mathrm{H}_{B}:(1,1) \alpha_{1}=-1$, $(1,1) \alpha_{\rho}=-(1+R)^{2} / R,(1,1) \mu=0$, and $(1,1) \Phi_{j}=0$. Together with the restriction in $\mathrm{H}_{S}$, these restrictions imply a martingale difference sequence for stock returns. The restricted model is denoted $\mathrm{M}_{1 D S B}$. The test statistic for $\mathrm{H}_{B}$ is asymptotically $\chi^{2}(2 k-1)$ if tested against $\mathrm{M}_{1 D S}$ and $\chi^{2}(2 k)$ if tested against $\mathrm{M}_{1 D}$.

\section{Asymptotic analysis}

Under the bubble hypothesis the conditions for the Granger-Johansen representation (Nielsen 2010, Assumption 1) reduce as follows.

Assumption A Under the bubble hypothesis the parameters satisfy (2.22) with $R>$ 0 . In addition, the parameters satisfy

(i) The nonstationary characteristic roots of $X_{t}$ are at 1 or $1+R$.

(ii) $\operatorname{det}\left(\alpha_{1 \perp}^{\prime} \Psi_{1} \beta_{1 \perp}\right) \neq 0$ and $\operatorname{det}\left(\alpha_{\rho \perp}^{\prime} \Psi_{\rho} \beta_{\rho \perp}\right) \neq 0$ where

$$
\Psi_{1}=I_{2}+R^{-1} \alpha_{\rho} \beta_{\rho}^{\prime}-\sum_{j=1}^{k-2} \Phi_{j}, \quad \Psi_{\rho}=I_{2}-R^{-1} \alpha_{1} \beta_{1}^{\prime}-\sum_{j=1}^{k-2} \rho^{-j} \Phi_{j} .
$$

The basic building blocks for the asymptotic theory are given by Lai and Wei (1985) and later adaptations by Nielsen (2005) to the case with deterministic terms. While the likelihood funtion is based on the assumption of independent, normal innovations the assumptions to the innovations can be relaxed in the asymptotic theory. The relaxed assumptions have to be formulated with two types of results in mind. On the one hand it is needed that the main component of the explosive common trend, $\sum_{s=1}^{t} \rho^{-s} \varepsilon_{s}$, converges. The Marcinkiewicz-Zygmund Theorem, see Lai and Wei (1983), shows that this process converges to a random vector almost surely. It suffices to assume that $\left(\varepsilon_{t}, \mathcal{F}_{t}\right)$ is a martingale difference sequence for some filtration $\mathcal{F}_{t}$ satisfying the following condition.

Assumption B For some $\gamma>2$ it holds $\sup _{t} \mathrm{E}\left\{\left(\varepsilon_{t}^{\prime} \varepsilon_{t}\right)^{2+\gamma} \mid \mathcal{F}_{t-1}\right\}<\infty$ a.s. 
On the other hand it is necessary that the normalised random walk $T^{-1 / 2} \sum_{s=1}^{t} \varepsilon_{s}$ converges to a Brownian motion. The assumption of Chan and Wei (1988) to the conditional variance of $\varepsilon_{t}$ is adopted since Assumption B already bounds the conditional moments.

Assumption $\mathbf{C}$ Suppose $\mathrm{E}\left(\varepsilon_{t} \varepsilon_{t}^{\prime} \mid \mathcal{F}_{t-1}\right)=\Omega$ a.s. where $\Omega$ is positive definite.

Under these assumptions it can be shown that the likelihood ratio test statistic for the explosive rational bubble model $\mathrm{M}_{1 D S B}$ against $\mathrm{M}_{1 D}$ is asymptotically $\chi^{2}$. The test statistic can be decomposed as

$$
\operatorname{LR}\left(\mathrm{H}_{B}, \mathrm{H}_{S} \mid \mathrm{M}_{1 D}\right)=\mathrm{LR}\left(\mathrm{H}_{A} \mid \mathrm{M}_{1 D}\right)+\mathrm{LR}\left(\mathrm{H}_{B} \mid \mathrm{M}_{1 D A}\right),
$$

where $\mathrm{H}_{A}$ is the hypothesis related to the extended cointegration vector $\left(\beta_{1}^{\prime}, \delta\right)=$ $\left(1,-R^{-1}, 0\right)$. Accordingly the first statistic relates to the restrictions on the cointegration vectors while the second statistic relates to the restrictions on the adjustment vectors $\alpha_{1}, \alpha_{\rho}, \Phi_{j}$. In the asymptotic analysis it is found that $\operatorname{LR}\left(\mathrm{H}_{B}, \mathrm{H}_{S} \mid \mathrm{M}_{1 D}\right)$ is the sum of two components: one involving a mixed Gaussian term and one involving a central limit theorem. This corresponds to the results for the I(1)-model, see Johansen (1995, §13), but the proof is somewhat more complicated.

Theorem 4.1 Suppose model $\mathrm{M}_{1 D S B}$ is satisfied. Assume A, B, C. Then the test statistic $\operatorname{LR}\left(\mathrm{H}_{B}, \mathrm{H}_{S} \mid \mathrm{M}_{1 D}\right)$ is asymptotically $\chi^{2}(2 k)$.

The proof is given in the appendix. It is related to the analysis in Nielsen (2010). Because the model $\mathrm{M}_{1 D S B}$ is analysed by regression the likelihood function can be analysed in a different way that gives rise to stronger results than in the above paper. Indeed, a global consistency result can be formulated.

The estimators in the model $\mathrm{M}_{1 D S B}$ are asymptotically normal. The estimator for $R$ converges at an exponential rate $(1+R)^{T}$ while the adjustment coefficients are standard $T^{1 / 2}$-consistent. Theorem B.11 in the appendix gives a precise statement.

\section{Empirical illustration: The US stock market}

We apply the methods to the annual US stock price and dividend series tabulated by Robert J. Shiller and available at www.robertshiller.com. Here, $P_{t}$ is the real S\&P Composite stock price index at January at year $t$, and $D_{t}$ denotes the associated real dividends paid during year $t-1$. These data have been used in numerous previous studies, including many of the earlier empirical bubble studies mentioned in the introduction (and in section 6 below). 

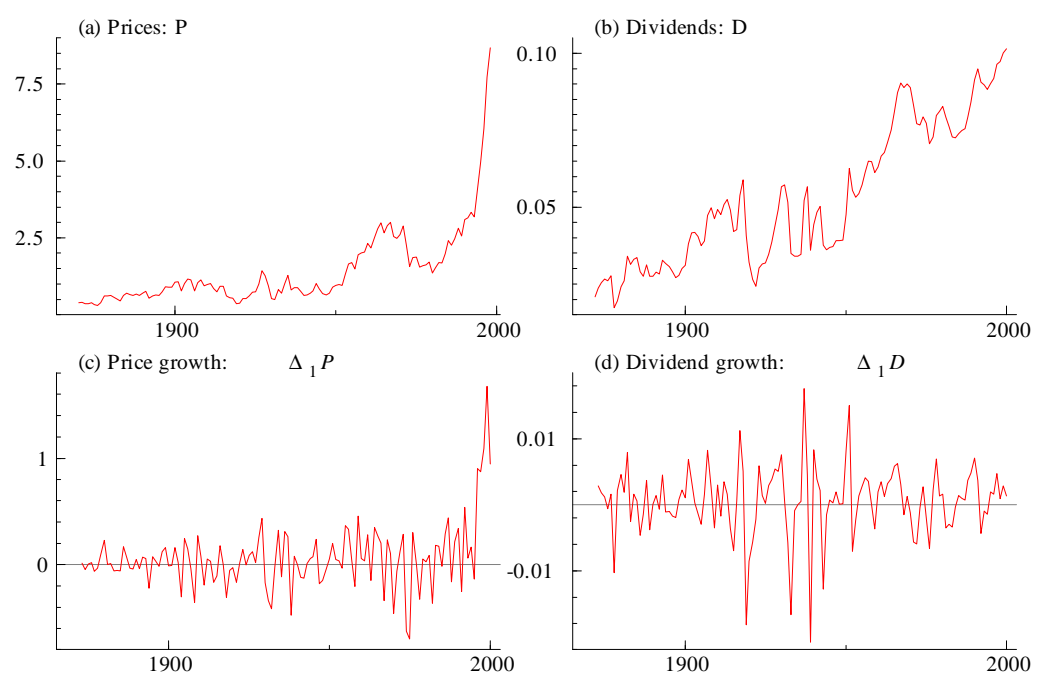

Figure 1: Data for prices, $P_{t}$, and dividends, $D_{t}$, and their differences. Note the exponential behaviour of $P_{t}$ and $\Delta_{1} P_{t}$ and the lack of exponential behaviour for dividends.

Figure 1 shows prices and dividends in levels and differences over the full sample period. An exponential pattern is seen both for levels and differences of $P_{t}$ while $D_{t}$ appears not to have exponential growth. The dramatic price increases that took place during the 1990s, and which have led many to argue that a bubble was driving the market in that period, are clearly seen in the figure. Shiller (2000), however, argues that not just the 1990s, but also some earlier periods (e.g. the 1920s and 1960s) were driven by speculative bubbles. We will therefore analyse both the full sample period 1872-2000 as well as a more recent sub-sample, 1974-2000. The full sample analysis serves mainly as an illustration as the empirical model is misspecified for the full sample, presumably due to the multiple bubbles. The sub-sample analysis is more substantial as it has one dominant bubble and the empirical model is well-specified.

The software OxMetrics by Doornik and Hendry (2001) is used for the analysis.

\subsection{Full sample period, 1872-2000}

Here the full sample is analysed using the procedure summarised in $§ 3.5$. The initial cointegration analysis is similar to that reported by Engsted (2006).

The first step is to fit a bivariate unrestricted vector-autoregression for $X_{t}=\left(P_{t}\right.$, $\left.D_{t}\right)^{\prime}$. The Hannan-Quinn criterion picks a two-lag model. Table 1 shows specification 


\begin{tabular}{|c|c|c|c|c|}
\hline Test & $P_{t}$ & $D_{t}$ & Test & system \\
\hline$\chi_{\text {normality }}^{2}(2)$ & $\begin{array}{c}14.9 \\
{[<0.001]}\end{array}$ & $\underset{[<0.001]}{24.7}$ & $\chi_{\text {normality }}^{2}(4)$ & $\begin{array}{c}39.5 \\
{[<0.001]}\end{array}$ \\
\hline$F_{a r, 1-2}(2,120)$ & $\begin{array}{l}1.6 \\
{[0.21]}\end{array}$ & $\begin{array}{l}2.7 \\
{[0.07]}\end{array}$ & $F_{a r, 1-2}(8,234)$ & $\begin{array}{l}1.7 \\
{[0.10]}\end{array}$ \\
\hline$F_{\text {arch }, 1-1}(1,120)$ & $\begin{array}{c}16.7 \\
{[<0.001]}\end{array}$ & $\begin{array}{l}1.2 \\
{[0.28]}\end{array}$ & & \\
\hline
\end{tabular}

Table 1: Specification tests for the unrestricted vector autoregression with 2 lags. US 1871 - 2000. All tests are asymptotically valid regardless of the location of the parameters. For the normality tests see Engler and Nielsen (2009), for autoregressive tests see Nielsen (2006a,b), for ARCH test see Doornik and Hendry (2001). p-values in square brackets.

\begin{tabular}{llll}
\hline Hypothesis & Likelihood & Test statistic & $p$-value \\
\hline$r \leq 2$ & 494.19 & & \\
$r \leq 1$ & 492.25 & $L R(r \leq 1 \mid M)=3.9$ & 0.44 \\
$r=0$ & 480.80 & $L R(r=0 \mid M)=26.8$ & 0.004 \\
\hline
\end{tabular}

Table 2: Cointegration rank determination with constant restricted to cointegration space. Critical values based on Johansen (1995, Table 15.2) and Doornik (1998).

tests for this model. While the specification appears to capture the autocorrelation in the data, it fails with respect to normality and autocorrelation in the squared residuals. Various recursive tests not shown here also indicate that the model parameters may not be stable over time. In the subsequent section 5.2 we analyse a sub-samble without this specification problem. Nonetheless, for comparison with existing results in the literature we do not attempt to obtain a better specified model for the full sample, and continue with the model as it is.

The four characteristic roots of the unrestricted vector autoregression, $\mathrm{M}$, are $1.183,0.9404$ and $0.1103 \pm 0.1904 i$. The largest root is clearly above one, and the second largest root is below - but close to - one. The unrestricted maximum likelihood value is 494.19 , see Table 2 .

The cointegration rank can be determined through Johansen's rank test with a constant restricted to the cointegration space. This test applies even with an explosive root. The results are reported in Table 2 . The restricted model $M_{1}$ with no cointegration, $r=0$, is firmly rejected, whereas the hypothesis of at most one cointegrating relation, $r \leq 1$ cannot be rejected, with a maximum likelihood value of 492.25 . Thus a single unit root appears present as required in the theory model, meaning that prices and dividends are 'cointegrated' in the sense that they share an I(1) trend. Imposing a unit root as in model $\mathrm{M}_{1}$ changes only slightly the largest characteristic root, to $\hat{\rho}=1.190$. The estimated cointegrating vector is $\hat{\beta}_{1}=(1,-22.2)$. This implies 


\begin{tabular}{llllll}
\hline Model & Hypothesis & Likelihood & Test statistic & d.f. & $p$-value \\
\hline$M_{1}$ & $H_{1}, r=1$ & 492.25 & & & \\
$M_{1 D}$ & $H_{1}, H_{D}$ & 491.93 & $L R\left(H_{D} \mid M_{1}\right)=0.64$ & 1 & 0.42 \\
$M_{1 D S}$ & $H_{1}, H_{D}, H_{S}$ & 491.12 & $L R\left(H_{S} \mid M_{1 D}\right)=1.62$ & 1 & 0.20 \\
& & & $L R\left(H_{S} \mid M_{1}\right)=2.26$ & 2 & 0.32 \\
$M_{1 D S B}$ & $H_{1}, H_{D}, H_{S}, H_{B}$ & \multirow{2}{*}{485.23} & $L R\left(H_{B} \mid M_{1 D S}\right)=11.80$ & 3 & 0.008 \\
& & & $L R\left(H_{B} \mid M_{1 D}\right)=13.40$ & 4 & 0.009 \\
& & & $L R\left(H_{B} \mid M_{1}\right)=14.04$ & 5 & 0.015 \\
\hline
\end{tabular}

Table 3: Tests of the rational bubble restrictions. See section 3.4 for a description of the various models and hypotheses.

an expected annual real return of $\hat{R}=1 / 22.2=4.5 \%$. This value is quite low and $1+\hat{R}=1.045$ deviates somewhat from the explosive root estimate. Below we test whether they are statistically different.

The next step is to test the hypothesis $\mathrm{H}_{D}$, i.e. that the co-explosive vector takes the form $\beta_{\rho}^{\prime}=(0,1)$ implying that dividends are non-explosive. The model here is $\mathrm{M}_{1 D}$ in (3.4) which for a given $\rho$ is estimated by reduced rank regression, c.f. section 3.4.1. The maximum likelihood estimate of $\rho$ is then obtained by a grid search over $\rho$ with $\hat{\rho}=1.192$. The estimated cointegrating vector is $\hat{\beta}_{1}=(1,-20.7)$ with an implied expected annual real return of $\hat{R}=4.9 \%$. The maximum likelihood value is 491.93 , and the $\chi^{2}(1)$ test statistic for the hypothesis $\mathrm{H}_{D}$ is 0.64 with a p-value of 0.42 , see Table 3. Thus, we cannot reject that dividends are non-explosive.

The third step is to test the hypothesis $\mathrm{H}_{S}$ which relates $R$ in the cointegrating vector to the explosive root $\rho$, i.e. $\beta_{1}=(1,-1 / R)^{\prime}$ and $\rho=1+R$, see section 3.4.2. The model here is $\mathrm{M}_{1 D S}$ as in (3.5). The explosive root is estimated by $\hat{\rho}=1.138$ implying an expected annual real stock return of $R=13.8 \%$. The likelihood value is 491.12. The test statistic for the hypothesis $\mathrm{H}_{S}$ is 1.62 with a p-value of 0.20 if tested against $\mathrm{M}_{1 D}$ and 2.26 with a p-value of 0.32 in the $\chi^{2}(2)$ distribution if tested against $\mathrm{M}_{1}$. Thus, despite the estimated difference between $R$ and $\rho-1$ seen above, they are not statistically different.

The estimated system (3.5) under $\mathrm{M}_{1 D S}$ with $\hat{R}=0.138$ is (with standard errors in parentheses):

$$
\begin{aligned}
& \Delta_{1} \Delta_{\rho} P_{t}=\underset{(0.088)}{-0.856}\left(\Delta_{\rho} S_{t-1}+\underset{(0.024)}{0.083)}-\underset{(4.4)}{14.0 \Delta_{1}} D_{t-1},\right. \\
& \Delta_{1} \Delta_{\rho} D_{t}=\underset{(0.0016)}{-0.0086}\left(\Delta_{\rho} S_{t-1}+\underset{(0.024)}{0.083)}-\underset{(0.080)}{0.926 \Delta_{1}} D_{t-1},\right. \\
& \hat{\sigma}_{P P}=0.261, \quad \hat{\sigma}_{D D}=0.00475, \quad \text { corr }=-0.0064 .
\end{aligned}
$$

The last line reports the estimated residual standard deviations for the two equations along with the residual correlation. As seen, the estimated adjustment coefficients are 
$\widehat{\alpha}_{1}=\left(\widehat{\alpha}_{1, P}, \widehat{\alpha}_{1, D}\right)^{\prime}=(-0.856,-0.0086)^{\prime}$ and $\widehat{\alpha}_{\rho}=\left(\widehat{\alpha}_{\rho, P}, \widehat{\alpha}_{\rho, D}\right)^{\prime}=(-14.0,-0.926)^{\prime}$, all highly statistically significant. As also seen, these unrestricted estimates are not wildly at odds with the restrictions in $(2.22)$, that is, $(1,1) \alpha_{1}=-1$ and $(1,1) \alpha_{\rho}=$ $-(1+R)^{2} / R=-9.38$ (for $R=0.138$ ), whereas the constraint $(1,1) \mu=0$ appears invalid. In the final step we explicitly test these restrictions.

The last step is to test the bubble hypothesis $\mathrm{H}_{B}$. In the restricted model $\mathrm{M}_{1 D S B}$ the explosive root is estimated by $\hat{\rho}=1.102$ implying an expected annual real stock return of $R=10.2 \%$. The likelihood value is 485.23. The test statistics for the hypothesis $\mathrm{H}_{B}$ are 11.80 (p-value: 0.008 ) in $\mathrm{M}_{1 D S}$ and 14.04 (p-value: 0.015 ) in $\mathrm{M}_{1}$. Thus the stock price model with a rational bubble is not supported by the data.

The problem in rejecting the bubble hypothesis lies primarily with the restriction to the constant and to some extent with the restriction to $\alpha_{1}$. We tried to impose the restrictions $(1,1) \alpha_{\rho}=-(1+R)^{2} / R,(1,1) \alpha_{1}=-1$, and $(1,1) \mu=0$ individually on the model $\mathrm{M}_{1 D S}$. This gave test statistics of 1.50 (p-value: 0.22 ), 2.88 (p-value: 0.09 ) and 4.34 (p-value: 0.04 ), respectively, in a $\chi^{2}(1)$ distribution. Imposing both $(1,1) \alpha_{1}=-1$ and $(1,1) \alpha_{\rho}=-(1+R)^{2} / R$, but leaving the restricted constant unconstrained, gave a test statistic of 5.08 (p-value: 0.08 ).
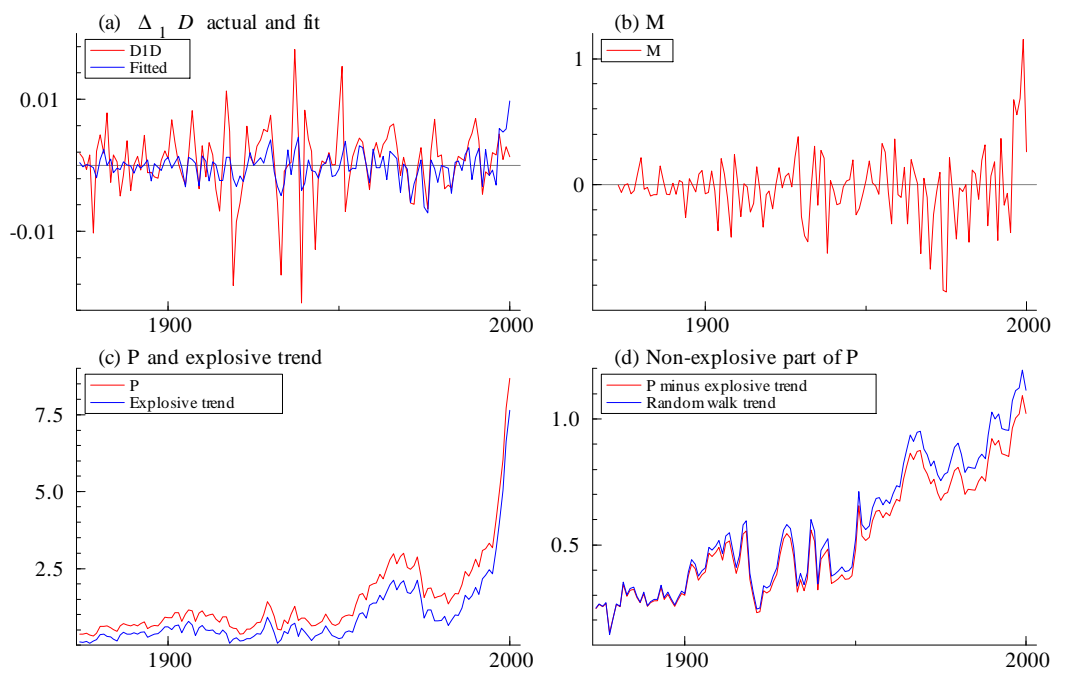

Figure 2: Results from $\mathrm{M}_{1 D S B}$ applied to full sample. (a) shows $\Delta_{1} D_{t}$ and the fit note that fit drifts towards the end. (b) shows $M_{t}$ - note drifting mean after 1960. (c) shows $P_{t}$ and the estimated explosive trend. $(d)$ shows $P_{t}$ minus explosive trend along with random walk trend.

It is interesting to consider graphical properties of the rejected bubble model 
$M_{1 D S B}$. The equations (3.6), (3.7) for the martingale difference $M_{t}$ and dividend growth $\Delta_{1} D_{t}$ were fitted. The drifting tendencies in panels $(a)$ and $(b)$ of Figure 2 contribute to the rejection of $\mathrm{H}_{B}$. Panel (c) shows the actual prices $P_{t}$ and the explosive trend for $P_{t}$ computed using the formula $(\rho-1)^{-1}(1,0) C_{\rho} \rho^{t}\left(A_{\rho}+\sum_{s=1}^{t} \rho^{-s} \hat{\varepsilon}_{s}\right)$ given in Theorem A.1. This explosive trend is the estimated bubble component in the model. We see that the model implies that the bubble contributes with a substantial part of the movements in prices over time. Panel $(d)$ compares the non-explosive part of $P_{t}$, that is the difference between $P_{t}$ and the explosive trend, with the random walk trend $(1-\rho)^{-1}(1,0) C_{1}\left(A_{1}+\sum_{s=1}^{t} \hat{\varepsilon}_{s}\right)$, see Theorem A.1.

The conclusion from the above results is that US stock prices are explosive while dividends are non-explosive, and the two variables have a common stochastic I(1) trend. The over-identifying restrictions implied by a standard stock price model with a constant discount rate and a rational bubble are however not supported at standard significance levels, implying that excess returns do not behave as a martingale difference. However, since the specification tests in Table 1 show evidence of misspecification, we are reluctant to interpret these results as providing strong evidence

against the standard stock price model. We consider the results indicative of the presence of a bubble.

\subsection{Sub-sample, 1974-2000}

In this section we will analyse a smaller sub-sample that contains data for 1976-2000, using the 1974 and 1975 observations as initial observations in the estimation of the vector autoregression. By letting the sample start in the 1970s we are also able to focus on the recent "bubble period" that ended in 2000 and - according to Shiller (2000) - began to build up already from the beginning of the 1980s. The proposed method is designed to work well for such a single bubble period.

Tables 4 and 5 report, respectively, specification tests for an unrestricted model with two lags and tests for the cointegration rank. There is no evidence of misspecification so the unrestricted vector autoregression seems to be econometrically well-specified. The characteristic roots are $1.258,0.675 \pm 0.354 i$, and -0.295 , thus clearly indicating an explosive root in the system. The rank tests point to a cointegration rank of unity, although the rejection of the hypothesis of no cointegration is marginal. Imposing a unit root changes only slightly the largest root, to 1.223. In the following we will assume the presence of a unit root.

The estimated cointegration vector is $\beta_{1}^{\prime}=(1,29.296)$. As seen, the coefficient to $D_{t}$ has the 'wrong' sign implying a negative expected return. However, inspection of the likelihood function reveals that it is extremely flat around the optimum; and tests of the hypotheses that either $(1,0)$ or $(0,1)$ are contained in the cointegration space cannot be rejected at the $5 \%$ level of significance, which implies that we can 


\begin{tabular}{lcclc}
\hline Test & $P_{t}$ & $D_{t}$ & Test & system \\
\hline$\chi_{\text {normality }}^{2}(2)$ & 0.6 & 1.7 & $\chi_{\text {normality }}^{2}(4)$ & 2.0 \\
$F_{\text {ar }, 1-2}(2,18)$ & {$[0.73]$} & {$[0.42]$} & & {$[0.74]$} \\
$F_{\text {arch }, 1-1}(1,23)$ & 0.1 & 1.4 & $F_{\text {ar }, 1-2}(8,30)$ & 1.4 \\
& 0.35 & {$[0.26]$} & & {$[0.22]$} \\
& {$[0.58]$} & {$[0.44]$} & & \\
\hline
\end{tabular}

Table 4: Specification tests for the unrestricted vector autoregression with 2 lags. US 1973 - 2000. All tests are asymptotically valid regardless of the location of the parameters. For the normality tests see Engler and Nielsen (2007), for autoregressive tests see Nielsen (2006a,b), for ARCH test see Doornik and Hendry (2007). p-values in square brackets.

\begin{tabular}{llll}
\hline Hypothesis & Likelihood & Test statistic & $p$-value \\
\hline$r \leq 2$ & 108.71 & & \\
$r \leq 1$ & 105.91 & $L R(r \leq 1 \mid M)=5.6$ & 0.23 \\
$r=0$ & 98.21 & $L R(r=0 \mid M)=21.1$ & 0.04 \\
\hline
\end{tabular}

Table 5: Cointegration rank determination with constant restricted to cointegration space. US 1973 - 2000. Critical values based on Johansen (1995, Table 15.2) and Doornik (1998).

treat either $P_{t}$ or $D_{t}$ as having no unit root. Thus, unfortunately the data are not very informative about the value of the expected return parameter $R$.

Table 6 reports the various tests associated with the bubble hypothesis. First, the $\mathrm{H}_{D}$ hypothesis that dividends are non-explosive gives a p-value of 0.99 while $\hat{\rho}=1.224$ is nearly unchanged. This is as expected from Figure 1 which clearly shows that the explosive root in the system belongs to $P_{t}$ and not $D_{t}$. Second, the $\mathrm{H}_{S}$ hypothesis that $\beta_{1}^{\prime}=(1,-1 / R)$ with $\rho=1+R$, gives a p-value of 0.57 when tested against $\mathrm{M}_{1 D}$ and $\widehat{\rho}=1.263$, which implies $R=26.1 \%$, clearly not an economically reasonable estimate of the expected annual return.

Testing the final hypothesis, $\mathrm{H}_{B}$, gives a $\mathrm{p}$-value of 0.07 if tested against model $\mathrm{M}_{1 D S}$, and a $\mathrm{p}$-value of 0.19 if tested against model $\mathrm{M}_{1}$. The decision not to reject the hypothesis $\mathrm{H}_{B}$ is marginal against $\mathrm{M}_{1 D S}$ albeit more convincing against $\mathrm{M}_{1}$, so all in all the sub-sample is more supportive of the standard stock market model than the full sample. As for the full sample the difficulties arise from the constant. Imposing only $(1,1) \alpha_{1}=-1$ and $(1,1) \alpha_{\rho}=-(1+R)^{2} / R$ but leaving the restricted constant unconstrained gives a likelihood of 104.68 , so a test statistic of 2.20 (p-value: 0.33 ) against $\mathrm{M}_{1 D S}$. Thus the test statistic for $\mathrm{M}_{1 D S B}$ against this intermediate hypothesis is 4.89 (p-value: 0.027 ).

Proceeding with the model $\mathrm{M}_{1 D S B}$ we note that the estimate of the explosive root 


\begin{tabular}{llllll}
\hline Model & Hypothesis & Likelihood & Test statistic & d.f. & $p$-value \\
$M_{1}$ & $H_{1}, r=1$ & 105.91 & & & \\
$M_{1 D}$ & $H_{1}, H_{D}$ & 105.91 & $L R\left(H_{D} \mid M_{1}\right)=0.0002$ & 1 & 0.99 \\
$M_{1 D S}$ & $H_{1}, H_{D}, H_{S}$ & 105.75 & $L R\left(H_{S} \mid M_{1 D}\right)=0.32$ & 1 & 0.57 \\
& & & $L R\left(H_{S} \mid M_{1}\right)=0.32$ & 2 & 0.85 \\
$M_{1 D S B}$ & $H_{1}, H_{D}, H_{S}, H_{B}$ & \multirow{2}{*}{102.20} & $L R\left(H_{B} \mid M_{1 D S}\right)=7.10$ & 3 & 0.07 \\
& & & $L R\left(H_{B} \mid M_{1 D}\right)=7.40$ & 4 & 0.12 \\
& & & $L R\left(H_{B} \mid M_{1}\right)=7.40$ & 5 & 0.19 \\
\hline
\end{tabular}

Table 6: Tests of the rational bubble restrictions. US 1973 - 2000. See section 3.4 for a description of the various models and hypotheses.

now becomes $\widehat{\rho}=1.156$ (standard error: 0.023 ) implying $\hat{R}=15.6 \%$ which is lower than before but still quite high. Here the standard error is computed from the second derivative of the profile likelihood and is valid under the normality assumption which is not rejected. The estimated model under $\mathrm{M}_{1 D S B}$ can be presented in two ways. First, the system representation is

$$
\begin{aligned}
\Delta_{1} \Delta_{\rho} P_{t} & =\underset{(0.0014)}{-1.0032} \Delta_{\rho} S_{t-1}-\underset{(0.16)}{7.92} \Delta_{1} D_{t-1} \\
\Delta_{1} \Delta_{\rho} D_{t} & =\underset{(*)}{0.0032 \Delta_{\rho}} S_{t-1}-\underset{(*)}{0.65 \Delta_{1} D_{t-1}} \\
\hat{\sigma}_{P P} & =0.362, \quad \hat{\sigma}_{D D}=0.00292, \quad \text { corr }=-0.262,
\end{aligned}
$$

while the martingale representation, as in (3.6) and (3.7), is

$$
\begin{aligned}
M_{t} & =\hat{\varepsilon}_{M, t} \\
\Delta_{1} D_{t} & =\underset{(0.0015)}{0.0032 \Delta_{\rho} S_{t-1}}+\underset{(0.17)}{0.51 \Delta_{1} D_{t-1}}-\underset{(0.0017)}{0.0021} M_{t}+\hat{\varepsilon}_{D \cdot M, t}, \\
\hat{\sigma}_{M M} & =0.126, \quad \hat{\sigma}_{D D \cdot M}=0.00295, \quad \text { corr }=0 .
\end{aligned}
$$

Figure 3 shows a graph of $M_{t}$ along with graphs of actual and fitted values of $\Delta_{1} D_{t}$ as well as a break down of the explosive trend and the random walk trend as for Figure 2. Note the improved fit for the sub-sample as compared with the full sample.

The conclusion from this sub-sample analysis, 1974-2000, is that real stock prices clearly contain an explosive component, and the formal restrictions implied by the rational bubble model cannot be rejected statistically at conventional significance levels, although the test for the bubble hypothesis itself, $\mathrm{H}_{B}$ against $\mathrm{M}_{1 D S}$, is marginal. The analysis does provide a rather high value of the expected return parameter $R$. It is also found that two quite different models for the relation between prices and dividends are both consistent with the data: Either $P_{t}$ does share the unit root in $D_{t}$ (i.e. 'cointegration' between $P_{t}$ and $D_{t}$ ), or it does not. The data are simply not 

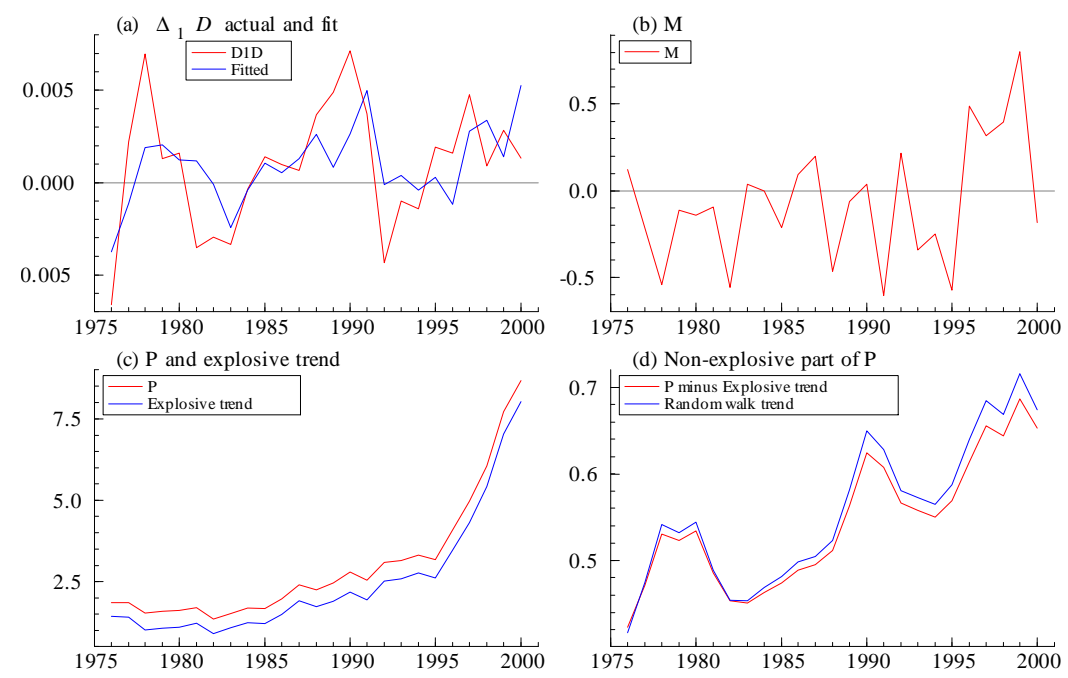

Figure 3: Results from $\mathrm{M}_{1 D S B}$ applied to sub-sample. (a) shows $\Delta_{1} D_{t}$ and the fit. (b) shows $M_{t}$. (c) shows $P_{t}$ and the estimated explosive trend. (d) shows $P_{t}$ minus explosive trend along with random walk trend.

able to discriminate between these two hypotheses. From an economic point of view (c.f. Shiller, 2000), and looking at figure 1, it is not totally unreasonable to consider prices during the 1980s and 1990s as being bubble-driven with no connection at all to fundamental variables like dividends.

\section{Comparing our test with earlier bubble tests}

In this section we briefly discuss our bubble testing procedure in relation to other bubble tests proposed in the literature. Earlier in the paper we discussed Diba and Grossman's (1988b) approach and, as we saw, in contrast to their analysis our approach makes it possible to estimate both the explosive root and the cointegrating vector between the $\mathrm{I}(1)$ components of $P_{t}$ and $D_{t}$, and it allows testing the restriction that a rational bubble imposes on the relation between the the explosive root and the cointegrating vector.

An important assumption in both Diba and Grossman and our analysis is that the discount factor, i.e. expected return, is constant. Craine (1993) modified Diba and Grossman's approach by showing that with a time-varying (but stationary) discount factor, the price-dividend ratio, $P_{t} / D_{t}$, should be stationary under no bubbles. The appealing feature of Craine's approach is that no unknown cointegrating vector has 
to be estimated. It suffices to just test the stationarity of $P_{t} / D_{t}$. On the other hand, no other testable restrictions follow from this approach. When we fit a first order autoregression to $P_{t} / D_{t}$ on our US 1872-2000 data, the autoregressive root is above one (1.0465) and the null hypothesis of a unit root against the one-sided explosive alternative is strongly rejected at the right tail of the Dickey-Fuller distribution (ADFvalue: 1.04$)$, consistent with the results in $\S 5$.

A potential weakness of cointegration based tests for bubbles is that they will have difficulties discovering periodically collapsing bubbles. Evans (1991) showed that in a finite sample unit root and cointegration tests will typically not identify the explosive component of periodically collapsing bubbles. Our co-explosive framework may not perform well in that situation. Indeed, the IT bubble is so dominant that explosiveness is picked up in a full sample ending in the years 1996 to 2000 but not if ending in 1995, thus supporting Evans' (1991) conjecture; see also discussion of Engsted (2006). When looking exclusively at the sub-sample from 1974 onward with a single bubble the explosiveness is, however, picked up. For instance for the sample ending in 1990 there is an explosive root of 1.10 .

In this paper we have confined attention to stochastic explosive bubbles driven by extraneous sources. We have not considered the kind of purely deterministic explosive bubbles analysed by Flood and Garber (1980), or the 'intrinsic' explosive bubble analysed by Froot and Obstfeld (1991) where the bubble is a non-linear deterministic function of fundamentals. For the kind of stochastic bubbles we analyse, West (1987) devised an often cited Hausman-type specification test, which compares two sets of estimates of the underlying asset pricing model. The first set of estimates is consistent both with and without a bubble, whereas the second set is only consistent in the absence of a bubble. The specification test then tests for equality of the two sets of estimates. Thus, the null hypothesis is no bubble, while the presence of a bubble should lead to rejection of the null hypothesis. One troublesome aspect of West's procedure (noted by West himself in West (1985), i.e. the working paper version of West (1987)), is that the test is not consistent: under the alternative hypothesis that bubbles are present, the probability that the test will reject the null of no bubbles will not go to unity asymptotically. The problem is directly a result of the explosiveness of prices under the alternative. The testing procedure we develop in the present paper does not face such problems because our null hypothesis explicitly involves bubbles.

Finally, in some recent literature (e.g. Cochrane, 2008; Balke and Wohar, 2009), bubbles are discussed within the log-linearized present value model of Campbell and Shiller (1988b). However, that model is based on a Taylor series expansion around the unconditional mean log dividend-price ratio which needs to be stationary in order for the log-linearization to be valid. Thus, that model in fact rules out bubbles from the outset. 


\section{Conclusions}

In this paper we have developed a procedure for testing a constant discount rate equity market model within a vector autoregressive framework. The special feature of our approach is that it allows for both unit roots in prices and dividends and an explosive root due to a rational speculative bubble. The analysis has been conducted within the co-explosive vector autoregressive framework of Nielsen (2010), which is an extension of the standard Johansen model for I(1) variables to the case with one explosive root in the system. We have developed likelihood ratio tests of various restrictions implied by the bubble model; these restrictions can be tested using standard $\chi^{2}$-inference.

In an empirical illustration using US stock market data that includes the "bubble period" in the 1990s, we find stong evidence of an explosive root in stock prices, while dividends do not contain explosive elements, thus lending support to the argument that US stock prices in part have been driven by a speculative bubble. For the full sample 1872-2000 the bubble restrictions are, however, rejected at standard significance levels - and this is when ignoring that the empirical model is mis-specified. For the sub-sample analysis for the period 1974-2000 the empirical model appears well-specified and the bubble restrictions cannot be rejected. 


\section{References}

Abreu, D. and Brunnermeier, M.K. (2003) Bubbles and crashes. Econometrica 71, 173-204.

Anderson, T.W. (1959) On asymptotic distributions of estimates of parameters of stochastic difference equations. Annals of Mathematical Statistics 30, 676-687.

Balke, N.S. and Wohar, M.E. (2009) Market fundamentals versus rational bubbles in stock prices: A Bayesian perspective. Journal of Applied Econometrics 24, $35-75$.

Bhargava, A. (1986) On the theory of testing for unit roots in observed time series. Review of Economic Studies 53, 369-384.

Bohl, M.T. (2003) Periodically collapsing bubbles in the US stock market? International Review of Economics and Finance 12, 385-397.

Boswijk, H.P. and Doornik, J.A. (2004) Identifying, estimating and testing restricted cointegrated systems: An overview. Statistica Neerlandica 58, 440-465.

Breusch, T.S. (1978) Testing for autocorrelation in dynamic linear models. Australian Economic Papers, 17, 334-355.

Brown, B.M. and Eagleson, G.K. (1971). Martingale convergence to infinitely divisible laws with finite variance. Transactions of the American Mathematical Society 162, 449-453.

Campbell, J.Y. and Shiller, R.J. (1987) Cointegration and tests of present value models. Journal of Political Economy 95, 1062-1088.

Campbell, J.Y. and Shiller, R.J. (1988a) Interpreting cointegrated models. Journal of Economic Dynamics and Control 12, 505-522.

Campbell, J.Y. and Shiller, R.J. (1988b) The dividend-price ratio and expectations of future dividends and discount factors. Review of Financial Studies 5, 243-280.

Chan, N.H. and Wei, C.Z. (1988) Limiting distributions of least squares estimates of unstable autoregressive processes. Annals of Statistics 16, 367-401.

Cochrane, J.H. (2008) The dog that did not bark: A defense of return predictability. Review of Financial Studies 21, 1533-1575.

Craine, R. (1993) Rational bubbles: A test. Journal of Economic Dynamics and Control 17, 829-846. 
Diba, B.T. and Grossman, H.I. (1988a) The theory of rational bubbles in stock prices. Economic Journal 98, 746-757.

Diba, B.T. and Grossman, H.I. (1988b) Explosive rational bubbles in stock prices. American Economic Review 78, 520-530.

Doornik, J. (1998) Approximations to the asymptotic distribution of cointegration tests. Journal of Economic Surveys 12, 573-593.

Doornik, J.A. and Hendry, D.F. (2001) Empirical econometric modelling using PcGive 10, vol. 1 and 2. London: Timberlake Consultants Press.

Engle, R. F. (1982). Autoregressive conditional heteroscedasticity, with estimates of the variance of United Kingdom inflation. Econometrica 50, 987-1007.

Engle, R.F., Hendry, D.F. and Richard, J.-F. (1983) Exogeneity. Econometrica 51, 277-304.

Engler, E. and Nielsen, B. (2009) The empirical process of autoregressive residuals. Econometric Journal 12, 367-381.

Engsted, T. (2006) Explosive bubbles in the cointegrated VAR model. Finance Research Letters 3, 154-162.

Evans, G.W. (1991) Pitfalls in testing for explosive bubbles in asset prices. American Economic Review 81, 922-930.

Flood, R.P. and Garber, P.M. (1980) Market fundamentals versus price-level bubbles: The first tests. Journal of Political Economy 88, 745-770.

Froot, K.A. and Obstfeld, M. (1991) Intrinsic bubbles: he case of stock prices. American Economic Review 81, 1189-1214.

Godfrey, L.G. (1978) Testing against general autoregressive and moving average error models when the regressors include lagged dependent variables. Econometrica, 46, 1293-1301.

Hannan, E.J. and Quinn, B.G. (1979) The determination of the order of an autoregression. Journal of the Royal Statistical Society B41, 190-195.

Johansen, S. (1991) Estimation and hypothesis testing of cointegration vectors in Gaussian vector autoregressive models. Econometrica 59, 1551-1580.

Johansen, S. (1995) Likelihood-based inference in cointegrated vector autoregressive models. Oxford: Oxford University Press. 
Johansen, S. and Swensen, A.R. (1999) Testing exact rational expectations in cointegrated vector autoregressive models. Journal of Econometrics 93, 73-91.

Johansen, S. and Swensen, A.R. (2004) More on testing exact rational expectations in cointegrated vector autoregressive models: Restricted constant and linear term. Econometrics Journal 7, 389-397.

Lai, T.L. and C.Z. Wei (1983) A note on martingale difference sequences satisfying the local Marcinkiewicz-Zygmund condition. Bulletin of the Institute of Mathematics, Academia Sinica 11, 1-13.

Lai, T.L. and C.Z. Wei (1985) Asymptotic properties of multivariate weighted sums with applications to stochastic regression in linear dynamic systems. In P.R. Krishnaiah (ed.), Multivariate Analysis VI, pp. 375-393. Amsterdam: Elsevier Science Publishers.

Leroy, S.F. (1989) Efficient capital markets and martingales. Journal of Economic Literature 27, 1583-1621.

Leroy, S.F. (2004) Rational exuberance. Journal of Economic Literature 42, 783-804.

Nielsen, B. and Rahbek, A. (2000) Similarity issues in cointegration models. Oxford Bulletin of Economics and Statistics 62, 5-22.

Nielsen, B. (2001) The Asymptotic Distribution of Unit Root Tests of Unstable Autoregressive Processes. Econometrica 69, 211-219.

Nielsen, B. (2005) Strong consistency results for least squares statistics in general vector autoregressive models. Econometric Theory 21, 534-561.

Nielsen, B. (2006a) Correlograms for non-stationary autoregressions. To appear in Journal of the Royal Statistical Society B68, 707-720.

Nielsen, B. (2006b) Order determination in general vector autoregressions. In Ho, H.-C., Ing, C.-K., and Lai, T.L. (eds): Time Series and Related Topics: In Memory of Ching-Zong Wei. IMS Lecture Notes and Monograph Series, 52, 93-112.

Nielsen, B. (2008a) On the Explosive Nature of Hyper-Inflation Data. Economics: The Open-Access, Open-Assessment E-Journal 2, 2008-21.

Nielsen, B. (2008b) Singular vector autoregressions with deterministic terms: Strong consistency and lag order determination. Discussion paper, Nuffield College. 
Nielsen, B. (2010) Analysis of co-explosive processes. Econometric Theory 26, $882-$ 915.

Pagan, A.R. (1984) Model evaluation by variable addition. In D.F. Hendry and K.F. Wallis (eds.) Econometrics and Quantitative Economics. Oxford: Basil Blackwell.

Schwarz, G. (1978) Estimating the dimension of a model. Annals of Statistics, 6, $461-464$.

Shiller, R.J. (2000) Irrational Exuberance. Princeton University Press. Princeton NJ.

Shleifer, A. (2000) Inefficient Markets. An Introduction Behavioral Finance. Oxford University Press, Oxford.

Tirole, J. (1982) On the possibility of speculation under rational expectations. Econometrica 50, 1163-1181.

Tirole, J. (1985) Asset bubbles and overlapping generations. Econometrica 53, 14991528 .

West, K.D. (1985) A specification test for speculative bubbles. Working Paper, Princeton University.

West, K.D. (1987) A specification test for speculative bubbles. Quarterly Journal of Economics 102, 553-580. 


\section{A Appendix: Granger-Johansen representation}

In this appendix a detailed representation theorem is derived for the co-explosive equation

$$
\Delta_{1} \Delta_{\rho} X_{t}=\alpha_{1} \beta_{1}^{\prime} \Delta_{\rho} X_{t-1}+\alpha_{\rho} \beta_{\rho}^{\prime} \Delta_{1} X_{t-1}+\sum_{j=1}^{k-2} \Phi_{j} \Delta_{1} \Delta_{\rho} X_{t-j}+\alpha_{1} \delta^{\prime}+\varepsilon_{t}
$$

in which $\alpha_{1}, \beta_{1}, \alpha_{\rho}, \beta_{\rho} \in \mathbb{R}^{2}$ and $\delta^{\prime} \in \mathbb{R}$.

The following notation is used throughout the appendices: For a matrix $\alpha$ with full column rank let $\bar{\alpha}=\alpha\left(\alpha^{\prime} \alpha\right)^{-1}$ while $\alpha_{\perp}$ denotes a basis to the orthogonal complement of the span of $\alpha$ so $\alpha_{\perp}^{\prime} \alpha=0$ and $\left(\alpha, \alpha_{\perp}\right)$ is invertible.

Theorem A.1 Suppose Assumption A holds. Then it holds

$$
X_{t}=\frac{1}{1-\rho} C_{1}\left(A_{1}+\sum_{s=1}^{t} \varepsilon_{s}\right)+\frac{1}{\rho-1} C_{\rho} \rho^{t}\left(A_{\rho}+\sum_{s=1}^{t} \rho^{-s} \varepsilon_{s}\right)+Y_{t}-\frac{\bar{\beta}_{1} \delta^{\prime}}{1-\rho} .
$$

where $Y_{t}=\theta_{U} U_{t}$ and

$$
\begin{aligned}
\Psi_{x} & =I_{p}+\frac{\alpha_{y} \beta_{y}^{\prime}}{y-x}-\sum_{j=1}^{k-2} x^{-j} \Phi_{j} \\
C_{x} & =x \beta_{x \perp}\left(\alpha_{x \perp}^{\prime} \Psi_{x} \beta_{x \perp}\right)^{-1} \alpha_{x \perp}^{\prime}, \\
A_{x} & =\Psi_{x} \Delta_{y} X_{0}-\frac{\alpha_{y} \beta_{y}^{\prime}}{y-x} \Delta_{x} X_{0}+\sum_{j=1}^{k-2} \Phi_{j} \sum_{h=0}^{j-1} x^{h-j} \Delta_{x} \Delta_{y} X_{-h}, \\
\theta_{U} & =\left(G_{1, \rho}, G_{\rho, 1}, H_{1, \rho, 1}+H_{\rho, 1,1}, \ldots, H_{1, \rho, k-2}+H_{\rho, 1, k-2}\right), \\
U_{t-1} & =\left\{\left(\beta_{1}^{\prime} \Delta_{\rho} X_{t-1}+\delta^{\prime}\right)^{\prime},\left(\beta_{\rho}^{\prime} \Delta_{1} X_{t-1}\right)^{\prime}, \Delta_{1} \Delta_{\rho} X_{t-1}, \ldots, \Delta_{1} \Delta_{\rho} X_{t-k+2}\right\} \\
G_{x, y} & =-\frac{C_{y} \alpha_{x}}{(y-x)^{2}}-\frac{C_{x} \Psi_{x} \bar{\beta}_{x}}{x-y}+\frac{x \bar{\beta}_{x}}{x-y}, \quad H_{x, y, n}=\frac{C_{x} x^{n-1}}{x-y} \sum_{j=n}^{k-2} \Phi_{j} x^{-j} .
\end{aligned}
$$

The process $U_{t}$ satisfies a first order vector autoregression and can be given a stationary initial distribution.

Remark A.2 A similar result can be derived for the model with a linear trend

$$
\Delta_{1} \Delta_{\rho} X_{t}=\alpha_{1}\left(\beta_{1}^{\prime}, \delta^{\prime}\right)\left(\begin{array}{c}
\Delta_{\rho} X_{t-1} \\
(1-\rho) t
\end{array}\right)+\alpha_{\rho} \beta_{\rho}^{\prime} \Delta_{1} X_{t-1}+\sum_{j=1}^{k-2} \Phi_{j} \Delta_{1} \Delta_{\rho} X_{t-j}+\mu+\varepsilon_{t},
$$

see Nielsen (2010). Define coefficients $\tau_{c}, \tau_{l}$ by

$$
\begin{aligned}
\tau_{l} & =C_{1} \mu /(1-\rho)+\left(C_{1} \Psi_{1}-I_{p}\right) \bar{\beta}_{1} \delta_{1}^{\prime}, \\
\beta_{1}^{\prime} \tau_{c} & =\bar{\alpha}_{1}^{\prime}\left(\Psi_{1} C_{1}-I_{p}\right) \mu /(1-\rho)+\bar{\alpha}_{1}^{\prime} \Psi_{1}\left(C_{1} \Psi_{1}-I_{p}\right) \bar{\beta}_{1} \delta_{1}^{\prime}+\delta_{1}^{\prime} \rho /(1-\rho) .
\end{aligned}
$$


Then $\tilde{X}_{t}=X_{t}-\bar{\beta}_{1} \beta_{1}^{\prime} \tau_{c}-\tau_{l}$ t solves the homogeneous solution $(A .2)$. As a consequence

$$
X_{t}=\frac{1}{1-\rho} C_{1}\left(A_{1}+\sum_{s=1}^{t} \varepsilon_{s}\right)+\frac{1}{\rho-1} C_{\rho} \rho^{t}\left(A_{\rho}+\sum_{s=1}^{t} \rho^{-s} \varepsilon_{s}\right)+Y_{t}+\bar{\beta}_{1} \beta_{1}^{\prime} \tau_{c}+\tau_{l} t .
$$

Proof of Theorem A.1. Homogeneous equation. Let $\tilde{X}_{t}=X_{t}+\bar{\beta}_{1} \delta^{\prime} /(1-\rho)$. Insert $\Delta_{1} X_{t}=\Delta_{1} \tilde{X}_{t}$ and $\beta_{1}^{\prime} \Delta_{\rho} X_{t-1}=\beta_{1}^{\prime} \Delta_{\rho} \tilde{X}_{t}-\delta^{\prime}$ in (A.1) to see that $\tilde{X}_{t}$ solves

$$
\Delta_{1} \Delta_{\rho} \tilde{X}_{t}=\alpha_{1} \beta_{1}^{\prime} \Delta_{\rho} \tilde{X}_{t-1}+\alpha_{\rho} \beta_{\rho}^{\prime} \Delta_{1} \tilde{X}_{t-1}+\sum_{j=1}^{k-2} \Phi_{j} \Delta_{1} \Delta_{\rho} \tilde{X}_{t-j}+\varepsilon_{t}
$$

It suffices to find the representation for this homogeneous equation. For the general case subtract $\bar{\beta}_{1} \delta^{\prime} /(1-\rho)$ from the representation.

Some identities. Note first that

$$
\begin{aligned}
(y-x) \Delta_{x} \tilde{X}_{s-1} & =\Delta_{x}^{2} \tilde{X}_{s}-\Delta_{x} \Delta_{y} \tilde{X}_{s} \\
\sum_{h=0}^{j-1} x^{h} \Delta_{x}^{2} \Delta_{y} \tilde{X}_{s-h} & =\Delta_{x} \Delta_{y} \tilde{X}_{s}-x^{j} \Delta_{x} \Delta_{y} \tilde{X}_{s-j} .
\end{aligned}
$$

Representation for $\beta_{x \perp}^{\prime} \Delta_{y} \tilde{X}_{t}$. It is first argued that

$$
\begin{aligned}
x \beta_{x \perp} \bar{\beta}_{x \perp}^{\prime} \Delta_{y} \tilde{X}_{t}= & C_{x} x^{t}\left(A_{x}+\sum_{s=1}^{t} x^{-s} \varepsilon_{s}\right)+C_{x} \frac{\alpha_{y} \beta_{y}^{\prime}}{y-x} \Delta_{x} \tilde{X}_{t} \\
& -C_{x} \Psi_{x} \bar{\beta}_{x} \beta_{x}^{\prime} \Delta_{y} \tilde{X}_{t}-C_{x} \sum_{j=1}^{k-2} \Phi_{j} \sum_{h=0}^{j-1} x^{h-j} \Delta_{x} \Delta_{y} \tilde{X}_{t-h} .
\end{aligned}
$$

To get that expression pre-multiply the model equation by $\alpha_{x \perp}^{\prime}$ and insert the above identities to get

$$
\begin{aligned}
\alpha_{x \perp}^{\prime} \Delta_{x} \Delta_{y} \tilde{X}_{s}= & \alpha_{x \perp}^{\prime} \varepsilon_{s}+(y-x)^{-1} \alpha_{x \perp}^{\prime} \alpha_{y} \beta_{y}^{\prime}\left(\Delta_{x}^{2} \tilde{X}_{s}-\Delta_{x} \Delta_{y} \tilde{X}_{s}\right) \\
& +\alpha_{x \perp}^{\prime} \sum_{j=1}^{k-2} x^{-j} \Phi_{j}\left(\Delta_{x} \Delta_{y} \tilde{X}_{s}-\sum_{h=0}^{j-1} x^{h} \Delta_{x}^{2} \Delta_{y} \tilde{X}_{s-h}\right)
\end{aligned}
$$

Gather $\Delta_{x} \Delta_{y} \tilde{X}_{s}$-terms and use $\Psi_{x}=I_{p}+(y-x)^{-1} \alpha_{y} \beta_{y}^{\prime}-\sum_{j=1}^{k-2} x^{-j} \Phi_{j}$ to get

$$
\alpha_{x \perp}^{\prime} \Psi_{x} \Delta_{x} \Delta_{y} \tilde{X}_{s}=\alpha_{x \perp}^{\prime} \varepsilon_{s}+\alpha_{x \perp}^{\prime} \frac{\alpha_{y} \beta_{y}^{\prime}}{y-x} \Delta_{x}^{2} \tilde{X}_{s}-\alpha_{x \perp}^{\prime} \sum_{j=1}^{k-2} \Phi_{j} \sum_{h=0}^{j-1} x^{h-j} \Delta_{x}^{2} \Delta_{y} \tilde{X}_{s-h} .
$$

Multiply with $x^{-s}$ and sum over $s$ and multiply with $x^{t}$ to get

$$
\begin{aligned}
\alpha_{x \perp}^{\prime} \Psi_{x}\left(\Delta_{y} \tilde{X}_{t}-x^{t} \Delta_{y} \tilde{X}_{0}\right)= & \alpha_{x \perp}^{\prime} \sum_{s=1}^{t} x^{t-s} \varepsilon_{s}+\alpha_{x \perp}^{\prime} \frac{\alpha_{y} \beta_{y}^{\prime}}{y-x}\left(\Delta_{x} \tilde{X}_{t}-x^{t} \Delta_{x} \tilde{X}_{0}\right) \\
& -\alpha_{x \perp}^{\prime} \sum_{j=1}^{k-2} \Phi_{j} \sum_{h=0}^{j-1} x^{h-j}\left(\Delta_{x} \Delta_{y} \tilde{X}_{t-h}-x^{t} \Delta_{x} \Delta_{y} \tilde{X}_{-h}\right) .
\end{aligned}
$$


Pre-multiply $\Delta_{y} X_{t}$ with $I_{p}=\bar{\beta}_{x} \beta_{x}^{\prime}+\beta_{x \perp} \bar{\beta}_{x \perp}^{\prime}$, recall $A_{x}$ and rearrange to get

$$
\begin{aligned}
\alpha_{x \perp}^{\prime} \Psi_{x} \beta_{x \perp} \bar{\beta}_{x \perp}^{\prime} \Delta_{y} X_{t}= & \alpha_{x \perp}^{\prime} x^{t}\left(A_{x}+\sum_{s=1}^{t} x^{-s} \varepsilon_{s}\right)+\alpha_{x \perp}^{\prime} \frac{\alpha_{y} \beta_{y}^{\prime}}{y-x} \Delta_{x} \tilde{X}_{t} \\
& -\alpha_{x \perp}^{\prime} \Psi_{x} \bar{\beta}_{x} \beta_{x}^{\prime} \Delta_{y} \tilde{X}_{t}-\alpha_{x \perp}^{\prime} \sum_{j=1}^{k-2} \Phi_{j} \sum_{h=0}^{j-1} x^{h-j} \Delta_{x} \Delta_{y} \tilde{X}_{t-h} .
\end{aligned}
$$

The matrix $\alpha_{x \perp}^{\prime} \Psi_{x} \beta_{x \perp}$ is invertible by Assumption A, so pre-multiply with its inverse and then by $x \beta_{x \perp}^{\prime}$ to get (A.3).

Representation. Note the identity

$$
X_{t}=\frac{y \Delta_{x} \tilde{X}_{t}-x \Delta_{y} \tilde{X}_{t}}{y-x}=\frac{y\left(\beta_{y \perp} \bar{\beta}_{y \perp}^{\prime}+\bar{\beta}_{y} \beta_{y}^{\prime}\right) \Delta_{x} \tilde{X}_{t}}{y-x}+\frac{x\left(\beta_{x \perp} \bar{\beta}_{x \perp}^{\prime}+\bar{\beta}_{x} \beta_{x}^{\prime}\right) \Delta_{y} \tilde{X}_{t}}{x-y} .
$$

Thus, $\tilde{X}_{t}$ has the desired representation in which

$$
\begin{aligned}
Y_{t}= & \frac{C_{1}}{1-\rho}\left\{\frac{\alpha_{\rho} \beta_{\rho}^{\prime}}{\rho-1} \Delta_{1} X_{t}-\Psi_{1} \bar{\beta}_{1} \beta_{1}^{\prime} \Delta_{\rho} X_{t}-\sum_{h=0}^{k-3}\left(\sum_{j=h+1}^{k-2} \Phi_{j}\right) \Delta_{1} \Delta_{\rho} X_{t-h}\right\} \\
& +\frac{C_{\rho}}{\rho-1}\left\{\frac{\alpha_{1} \beta_{1}^{\prime}}{1-\rho} \Delta_{\rho} X_{t}-\Psi_{\rho} \bar{\beta}_{\rho} \beta_{\rho}^{\prime} \Delta_{1} X_{t}-\sum_{h=0}^{k-3} \rho^{h}\left(\sum_{j=h+1}^{k-2} \Phi_{j} \rho^{-j}\right) \Delta_{1} \Delta_{\rho} X_{t-h}\right\} \\
& +\frac{1}{1-\rho} \bar{\beta}_{1} \beta_{1}^{\prime} \Delta_{\rho} X_{t}+\frac{\rho}{\rho-1} \bar{\beta}_{\rho} \beta_{\rho}^{\prime} \Delta_{1} X_{t} .
\end{aligned}
$$

Rearrange the terms of $Y_{t}$ to get the desired expression for $Y_{t}$.

The process $U_{t}$. The model equation implies a first order vector autoregression for $U_{t}$. Assuming A this can be given a stationary initial distribution as stated in Nielsen (2010, Theorem 1). 


\section{B Appendix: Proof of asymptotic results}

In this appendix the asymptotic results are proved.

The abbreviations a.s., P and D are used for properties holding almost surely, in probability, and in distribution respectively.

\section{B.1 Notation and preliminary asymptotic results}

\section{B.1.1 Rotating the data vector}

A feature of the vector autoregressive setup is its invariance to linear transformations. In the main discussion of the results the vector $X_{t}=\left(P_{t}, D_{t}\right)^{\prime}$ is analysed. In the proofs it is convenient to choose $X_{t}$ in a different way. The issue is that the bubble hypothesis is that

$$
M_{t}=P_{t}+D_{t}-(1+R) P_{t-1}
$$

is a martingale difference where the contemporaneous component of $M_{t}$ is $P_{t}+D_{t}$. For the proof it is convenient to choose

$$
X_{t}=\left(\begin{array}{c}
P_{t}+D_{t} \\
D_{t}
\end{array}\right)=\left(\begin{array}{ll}
1 & 1 \\
0 & 1
\end{array}\right)\left(\begin{array}{c}
P_{t} \\
D_{t}
\end{array}\right)
$$

Accordingly the error term of the model equation is denoted

$$
\varepsilon_{t}=\left(\begin{array}{c}
\varepsilon_{M, t} \\
\varepsilon_{D, t}
\end{array}\right) \quad \text { with } \quad \Omega=\operatorname{Cov}\left(\varepsilon_{t}\right)=\left(\begin{array}{cc}
\sigma_{M M} & \sigma_{M D} \\
\sigma_{D M} & \sigma_{D D}
\end{array}\right)
$$

and the conditional error $\varepsilon_{D \cdot M, t}=\varepsilon_{M, t}-\omega \varepsilon_{D, t}$ where $\omega=\sigma_{D M} \sigma_{M M}^{-1}$. The spread is

$$
S_{t}=\beta_{1}^{\prime} X_{t}=P_{t}-R^{-1} D_{t}=\left(P_{t}+D_{t}\right)-\mathcal{G} D_{t} \quad \text { with } \quad \mathcal{G}=R^{-1}+1 .
$$

Accordingly the cointegrating and the coexplosive vectors for the rotated system are

$$
\beta_{1}=\left(\begin{array}{c}
1 \\
-\mathcal{G}
\end{array}\right), \quad \beta_{1 \perp}=\left(\begin{array}{c}
\mathcal{G} \\
1
\end{array}\right), \quad \beta_{\rho}=\left(\begin{array}{l}
0 \\
1
\end{array}\right), \quad \beta_{\rho \perp}=\left(\begin{array}{l}
1 \\
0
\end{array}\right)
$$

\section{B.1.2 The data generating process}

In the probabilistic analysis the properties of the likelihood function will be analysed for each parameter $\left(\vartheta^{\circ}, \Omega_{\circ}\right)$ satisfying the restricted model $\mathrm{M}_{1 D S B}$. Introduce the vector $\mathcal{S}_{t}^{\circ}=\left(U_{t}^{\circ \prime}, V_{t}^{\circ \prime}, W_{t}^{\circ \prime}\right)^{\prime}$ where

$$
\begin{aligned}
& U_{t}^{\circ}=\left\{\left(\beta_{1}^{\circ \prime} \Delta_{\rho_{\circ}} X_{t}\right)^{\prime},\left(\beta_{\rho}^{\circ \prime} \Delta_{1} X_{t}\right)^{\prime},\left(\Delta_{1} \Delta_{\rho_{\circ}} X_{t}\right)^{\prime}, \ldots,\left(\Delta_{1} \Delta_{\rho_{\circ}} X_{t-k+3}\right)^{\prime}\right\}^{\prime}, \\
& V_{t}^{\circ}=\beta_{1 \perp}^{\circ \prime} \Delta_{\rho_{\circ}} X_{t}, \quad W_{t}^{\circ}=\beta_{\rho \perp}^{\circ} \Delta_{1} X_{t}, \quad \mathcal{R}_{t}^{\circ}=\left(M_{t}^{\circ}, U_{t-1}^{\circ}\right)^{\prime} .
\end{aligned}
$$


The data generating process is

$$
M_{t}=\varepsilon_{M, t}^{\circ}, \quad \Delta_{1} D_{t}=\left(\omega^{\circ}, \theta^{\circ \prime}\right)^{\prime} \mathcal{R}_{t}+\varepsilon_{D \cdot M, t}^{\circ},
$$

where $\vartheta^{\circ}=\left(\omega^{\circ}, \theta^{\circ \prime}\right)^{\prime}$ and $\theta^{\circ \prime}=\left(\alpha_{1, D}^{\circ}, \alpha_{\rho, D}^{\circ}, \Phi_{1, D}^{\circ \prime}, \ldots, \Phi_{k-2, D}^{\circ \prime}\right)$.

\section{B.1.3 Some further parameters}

From Nielsen (2010) it is known that the analysis of the unrestricted model $M_{1 D}$ involves the parameters

$$
\tau_{\perp}^{\circ}=\Psi_{\rho}^{\circ} \bar{\beta}_{\rho \perp}^{\circ}, \quad \Psi_{\rho}^{\circ}=I_{p}+\frac{\Pi_{1}^{\circ}}{1-\rho_{\circ}}-\sum_{j=1}^{k-2} \rho_{\circ}^{-j} \Phi_{j}^{\circ},
$$

as well as the projection matrices

$$
\mathcal{P}_{\tau_{\perp}}^{\circ}=\tau_{\perp}^{\circ}\left(\tau_{\perp}^{\circ \prime} \Omega_{\circ}^{-1} \tau_{\perp}^{\circ}\right)^{-1} \tau_{\perp}^{\circ} \Omega_{\circ}^{-1}, \quad \mathcal{P}_{\alpha}^{\circ}=\alpha_{1}^{\circ}\left(\alpha_{1}^{\circ} \Omega_{\circ}^{-1} \alpha_{1}^{\circ}\right)^{-1} \alpha_{1}^{\circ} \Omega_{\circ}^{-1} .
$$

In the restricted model $\mathrm{M}_{1 D S B}$ the restrictions (2.22) imply

$$
\tau_{\perp}^{\circ}=\left(\begin{array}{c}
\mathcal{G}^{\circ} \\
-R_{\circ}^{-1} \alpha_{1, D}^{\circ}-\sum_{j=1}^{k-2}\left(1+R_{\circ}\right)^{-j} \Phi_{j, D P}^{\circ}
\end{array}\right)=\left(\begin{array}{cc}
1 & 0 \\
0 & -\theta^{\circ \prime}
\end{array}\right) \mathcal{H}^{\circ},
$$

where the coefficient $\mathcal{G}^{\circ}$ and the vector $\mathcal{H}^{\circ}$ are given by

$$
\mathcal{G}^{\circ}=R_{\circ}^{-1}+1, \quad \mathcal{H}^{\circ}=\left\{\mathcal{G}^{\circ}, R_{\circ}^{-1}, 0,\left(1+R_{\circ}\right)^{-1} \bar{\beta}_{\rho \perp}^{\circ}, \ldots,\left(1+R_{\circ}\right)^{2-k} \bar{\beta}_{\rho \perp}^{\circ}\right\}^{\prime} .
$$

\section{B.1.4 A preliminary asymptotic result}

It is convenient to recall the following asymptotic result stated as Lemma A.1 in Nielsen (2010), but derived from Chan and Wei (1988) and Nielsen (2005). Introduce the block diagonal normalisation matrix $N_{\mathcal{S}}=\operatorname{diag}\left(I_{\operatorname{dim} U}, N_{V}, N_{W}, 1\right)$ where

$$
N_{V}=\operatorname{diag}\left\{T^{-1 / 2} I_{p-r},(1-\rho)^{-1}\right\}, \quad N_{W}=T^{1 / 2} \rho_{\circ}^{-T} .
$$

Lemma B.1 Let $X_{t}$ satisfy $\mathrm{M}_{1 D S B}$ and be given by (B.7). Assume A, B, C. Let $\xi$ be a constant satisfying $\xi<\gamma /(2+\gamma)$, recalling $\gamma$ defined in Assumption $B$.

Define sample variances $\widehat{\operatorname{var}}\left(x_{t}\right)=T^{-1} \sum_{t=1}^{T} x_{t} x_{t}^{\prime}$. Then stochastic matrices $\Sigma_{W W}$, $\Sigma_{V V}, \Sigma_{S S}$ and a deterministic matrix $\Sigma_{U U}$ exist so

(i) $\widehat{\operatorname{var}}\left(\varepsilon_{t}^{\circ}\right) \stackrel{\text { a.s. }}{=} \Omega_{\circ}+\mathrm{o}\left(T^{-\xi}\right)+\mathrm{o}\left(T^{\eta-1 / 2}\right)$ for all $\eta>0$.

(ii) $\widehat{\operatorname{var}}\left(U_{t-1}^{\circ}\right) \stackrel{\text { a.s. }}{\rightarrow} \Sigma_{U U}>0$.

(iii) $\hat{\Sigma}_{W W}=\rho_{\circ}^{-2 T} \sum_{t=1}^{T}\left(W_{t-1}^{\circ}\right)^{2}=\rho_{\circ}^{-2 T} T \widehat{\operatorname{var}}\left(W_{t-1}^{\circ}\right) \stackrel{\text { a.s. }}{\rightarrow} \Sigma_{W W} \stackrel{\text { a.s. }}{>} 0$. 
(iv) $\hat{\Sigma}_{V V}=\widehat{\operatorname{var}}\left(N_{V} V_{t-1}^{\circ}\right) \stackrel{\mathrm{D}}{\rightarrow} \Sigma_{V V} \stackrel{\text { a.s. }}{>} 0$.

(v) $\widehat{\operatorname{var}}\left(N_{S} S_{t-1}^{\circ}\right) \stackrel{\mathrm{D}}{\rightarrow} \Sigma_{S S} \stackrel{\text { a.s. }}{>} 0$.

Define sample correlations $\widehat{\operatorname{corr}}\left(x_{t}, y_{t}\right)=\left(\sum_{t=1}^{T} x_{t}^{\otimes 2}\right)^{-1 / 2} \sum_{t=1}^{T} x_{t} y_{t}^{\prime}\left(\sum_{t=1}^{T} y_{t}^{\otimes 2}\right)^{-1 / 2}$, so

(vi) $\widehat{\operatorname{corr}}\left(S_{t-1}^{\circ}, \varepsilon_{t}^{\circ}\right) \stackrel{\text { a.s. }}{=} \mathrm{o}\left(T^{-\xi / 2}\right)$.

(vii) $\widehat{\operatorname{corr}}\left\{\left(U_{t-1}^{\circ \prime}, V_{t-1}^{\circ \prime}, 1\right)^{\prime}, \varepsilon_{t}^{\circ}\right\}=\mathrm{O}_{\mathrm{P}}\left(T^{-1 / 2}\right)$.

In addition it holds jointly for some stochastic matrices $\Sigma_{V \varepsilon}, \Sigma_{U \varepsilon}, \Sigma_{V U}$ that

(vii) $\hat{\Sigma}_{U \epsilon}=T^{-1 / 2} \sum_{t=1}^{T}\left(U_{t-1}^{\circ}\right) \varepsilon_{t}^{\circ \prime} \stackrel{\mathrm{D}}{\rightarrow} \Sigma_{U \varepsilon}$. and $\hat{\Sigma}_{U \epsilon} \stackrel{\text { a.s. }}{=} \mathrm{o}\left(T^{\eta}\right)$ for all $\eta>0$.

(viii) $\hat{\Sigma}_{V \epsilon}=T^{-1 / 2} \sum_{t=1}^{T} N_{V}\left(V_{t-1}^{\circ}\right) \varepsilon_{t}^{\circ \prime} \stackrel{\mathrm{D}}{\rightarrow} \Sigma_{V \varepsilon}$.

(ix) $\hat{\Sigma}_{W \epsilon}=\rho_{\circ}^{-T} \sum_{t=1}^{T} W_{t-1}^{\circ} \epsilon_{t}^{\circ} \stackrel{\text { a.s. }}{=} \mathrm{o}\left(T^{(1-\xi) / 2}\right)$.

\section{B.2 Some asymptotic results for a given value of $R$}

The proof will involve analysis of product sums involving $\mathcal{R}_{t}$ defined as

$$
\mathcal{R}_{t}=\left(M_{t}, U_{t-1}^{\prime}\right)^{\prime}, \quad U_{t}=\left\{\left(\beta_{1}^{\prime} \Delta_{\rho} X_{t}\right)^{\prime},\left(\beta_{\rho}^{\prime} \Delta_{1} X_{t}\right)^{\prime},\left(\Delta_{1} \Delta_{\rho} X_{t}\right)^{\prime}, \ldots,\left(\Delta_{1} \Delta_{\rho} X_{t-k+3}\right)^{\prime}\right\}^{\prime} .
$$

for some $\rho$. Some expansions of these product sums are needed. It is convenient to introduce the notation

$$
\begin{aligned}
\left(\begin{array}{cc}
S_{\varepsilon_{M} \varepsilon_{M}}^{\circ} & S_{\varepsilon_{M} W}^{\circ} \\
S_{W \varepsilon_{M}}^{\circ} & S_{W W}^{\circ}
\end{array}\right) & =\frac{1}{T} \sum_{t=1}^{T}\left(\begin{array}{c}
M_{t}^{\circ} \\
W_{t-1}^{\circ}
\end{array}\right)^{\otimes 2}, \\
\left(\begin{array}{ccc}
S_{D D \cdot M}^{\circ} & S_{\varepsilon_{D \cdot M} \mathcal{R}}^{\circ} & S_{\varepsilon_{D \cdot M} W}^{\circ} \\
S_{\mathcal{R} \varepsilon_{D} \cdot M}^{\circ} & S_{\mathcal{R} \mathcal{R}}^{\circ} & S_{\mathcal{R} W}^{\circ}
\end{array}\right) & =\frac{1}{T} \sum_{t=1}^{T}\left(\begin{array}{c}
\varepsilon_{D \cdot M, t}^{\circ} \\
\mathcal{R}_{t}^{\circ}
\end{array}\right)\left(\varepsilon_{D \cdot M, t}^{\circ}, \mathcal{R}_{t}^{\circ \prime}, W_{t-1}^{\circ}\right),
\end{aligned}
$$

the sum $S_{\Delta \Delta}=T^{-1} \sum_{t=1}^{T}\left(\Delta_{1} D_{t}\right)^{2}$ as well as the partial product sums

$$
\left(\begin{array}{cc}
S_{\mathcal{R} \mathcal{R} \cdot \Delta}^{\circ} & S_{\mathcal{R} W \cdot \Delta}^{\circ} \\
S_{W \mathcal{R} \cdot \Delta}^{\circ} & S_{W W \cdot \Delta}^{\circ}
\end{array}\right)=\left(\begin{array}{cc}
S_{\mathcal{R} \mathcal{R}}^{\circ} & S_{\mathcal{R} W}^{\circ} \\
S_{W \mathcal{R}}^{\circ} & S_{W W}^{\circ}
\end{array}\right)-\left(\begin{array}{c}
S_{\mathcal{R} \Delta}^{\circ} \\
S_{W \Delta}^{\circ}
\end{array}\right) S_{\Delta \Delta}^{-1}\left(S_{\Delta \mathcal{R}}^{\circ}, S_{\Delta W}^{\circ}\right) .
$$

Lemma B.2 Assume A, B, C. Define $\mathcal{D}_{R}=\left(R-R_{\circ}\right)$ and $\mathcal{I}_{R}=\left(R^{-1}-R_{\circ}^{-1}\right)$. Let $\mathrm{o}_{\text {pol }}=\mathrm{o}\left(T^{-k}\right)$ for some finite $k$ not depending on $R$. Then

$$
\begin{array}{cl}
\frac{1}{T} \sum_{t=1}^{T} \varepsilon_{D \cdot M, t}^{\circ} \mathcal{R}_{t}^{\prime} \stackrel{\text { a.s. }}{=} & S_{\varepsilon_{D \cdot M} \mathcal{R}}^{\circ}-\mathcal{D}_{R} S_{\varepsilon_{D \cdot M} W}^{\circ} \mathcal{H}^{\circ \prime}+\left(\mathcal{D}_{R}+\mathcal{I}_{R}\right) \mathrm{o}_{p o l}, \\
\frac{1}{T} \sum_{t=1}^{T} \mathcal{R}_{t}^{\circ} \mathcal{R}_{t}^{\prime} \stackrel{\text { a.s. }}{=} & S_{\mathcal{R} \mathcal{R}}^{\circ}-\mathcal{D}_{R} S_{\mathcal{R} W}^{\circ} \mathcal{H}^{\circ \prime}+\left(\mathcal{D}_{R}+\mathcal{I}_{R}\right) \mathrm{o}_{p o l}, \\
\frac{1}{T} \sum_{t=1}^{T} \mathcal{R}_{t} \mathcal{R}_{t}^{\prime} \stackrel{\text { a.s. }}{=} & S_{\mathcal{R} \mathcal{R}}^{\circ}-\mathcal{D}_{R}\left(S_{\mathcal{R} W}^{\circ} \mathcal{H}^{\circ \prime}+\mathcal{H}_{W \mathcal{R}}^{\circ}\right)+\mathcal{D}_{R}^{2} \mathcal{H}^{\circ} S_{W W}^{\circ} \mathcal{H}^{\circ \prime} \\
& +\left(\mathcal{D}_{R}+\mathcal{I}_{R}\right)\left(1+\rho_{\circ}^{T} \mathcal{D}_{R}+\mathcal{D}_{R}+\mathcal{I}_{R}\right) \mathrm{o}_{p o l} .
\end{array}
$$


In particular it holds

$$
\begin{aligned}
\frac{1}{T} \sum_{t=1}^{T} \varepsilon_{D \cdot M, t}^{\circ} M_{t}^{\prime} & \stackrel{\text { a.s. }}{=} S_{\varepsilon_{D \cdot M} \varepsilon_{M}}^{\circ}-\mathcal{D}_{R}\left(\mathcal{G}^{\circ} S_{\varepsilon_{D \cdot M} W}^{\circ}+\mathrm{o}_{p o l}\right) \\
\frac{1}{T} \sum_{t=1}^{T} M_{t}^{2} & \stackrel{\text { a.s. }}{=} S_{\varepsilon_{M} \varepsilon_{M}}^{\circ}-2 \mathcal{D}_{R}\left(\mathcal{G}^{\circ} S_{\varepsilon_{M} W}^{\circ}+\mathrm{o}_{p o l}\right)+\mathcal{D}_{R}^{2}\left\{\mathcal{G}^{\circ 2} S_{W W}^{\circ}+\mathrm{o}(1)\right\}(.)
\end{aligned}
$$

Proof of Lemma B.2. Identities. Recall the definition of $X$ in (B.2) and of the cointegrating and the coexplosive vectors in (B.4). Then it holds

$$
\left(\begin{array}{c}
\beta_{1}^{\prime} \Delta_{1+R} \\
\beta_{\rho}^{\prime} \Delta_{1} \\
\beta_{1 \perp}^{\prime} \Delta_{1+R} \\
\beta_{\rho \perp}^{\prime} \Delta_{1}
\end{array}\right) X_{t-1}=\left(\begin{array}{cccc}
1 & -\frac{1}{R} & -(1+R) & \frac{1+R}{R} \\
0 & 1 & 0 & -1 \\
1 & R & -(1+R) & -R(1+R) \\
1 & 0 & -1 & 0
\end{array}\right)\left(\begin{array}{c}
P_{t-1} \\
D_{t-1} \\
P_{t-2} \\
D_{t-2}
\end{array}\right)
$$

which has the solution

$$
\left(\begin{array}{c}
P_{t-1} \\
D_{t-1} \\
P_{t-2} \\
D_{t-2}
\end{array}\right)=\left(\begin{array}{cccc}
-R & 0 & -1 & 1+R \\
1 & 1+R & -R & 0 \\
-R & 0 & -1 & 1 \\
1 & 1 & -R & 0
\end{array}\right)\left(\begin{array}{c}
\frac{1}{1+R^{2}} \beta_{1}^{\prime} \Delta_{1+R} \\
\frac{1}{R} \beta_{\rho}^{\prime} \Delta_{1} \\
\frac{1}{R\left(1+R^{2}\right)} \beta_{1 \perp}^{\prime} \Delta_{1+R} \\
\frac{1}{R} \beta_{\rho \perp}^{\prime} \Delta_{1}
\end{array}\right) X_{t-1}
$$

It also holds, see Nielsen (2010, equations A.17, A.18), that

$$
\begin{aligned}
\Delta_{1} \Delta_{1+R} X_{t-j} & =\Delta_{1} \Delta_{1+R_{\circ}} X_{t-j} \\
& +\left(R_{\circ}-R\right)\left(1+R_{\circ}\right)^{-j}\left\{\Delta_{1} X_{t-1}-\sum_{l=1}^{j}\left(1+R_{\circ}\right)^{l-1} \Delta_{1} \Delta_{1+R_{\circ}} X_{t-l}\right\} .
\end{aligned}
$$

Expansion of $\mathcal{R}$. It is to be derived that $\mathcal{R}_{t}=\left(M_{t}, U_{t-1}^{\prime}\right)^{\prime}$, see (B.6), satisfies

$$
\mathcal{R}_{t}=\mathcal{R}_{t}^{\circ}-\mathcal{D}_{R} \mathcal{H}^{\circ} W_{t-1}^{\circ}-\left(\mathcal{D}_{R} \mathcal{H}_{2}+\mathcal{I}_{R} e_{2} \mathcal{H}_{3}^{\prime}\right)\left(U_{t-1}^{\circ \prime}, V_{t-1}^{\circ}\right)^{\prime}
$$

for some matrices $\mathcal{H}_{2} \in \mathbb{R}^{(2 k-2) \times(2 k-1)}$ and $\mathcal{H}_{3} \in \mathbb{R}^{2 k-1}$ not depending on $R$ and where $e_{2}=\left(0,1,0_{1 \times 2(k-2)}\right)^{\prime}$.

The expression for $M_{t}$. Since $M_{t}=P_{t}+D_{t}-(1+R) P_{t-1}$ by (B.1) then $M_{t}=$ $M_{t}^{\circ}-\mathcal{D}_{R} P_{t-1}$. Due to (B.14) then $P_{t-1}$ is the sum of $\left(R_{\circ}^{-1}+1\right) W_{t-1}^{\circ}$ and some linear combination of $U_{t-1}^{\circ}, V_{t-1}^{\circ}$.

The first coordinate of $U_{t-1}$ is $\beta_{1}^{\prime} \Delta_{1+R} X_{t-1}$. Using (2.4) write

$$
\beta_{1}^{\prime} \Delta_{1+R} X_{t-1}=\Delta_{1+R} S_{t-1}=M_{t-1}-\left(R^{-1}+1\right) \Delta_{1} D_{t-1} .
$$


Writing $M_{t-1}=M_{t-1}^{\circ}-\mathcal{D}_{R} P_{t-2}$ and adding and subtracting $R_{\circ}^{-1} \Delta_{1} D_{t-1}$ shows

$$
\beta_{1}^{\prime} \Delta_{1+R} X_{t-1}=\beta_{1}^{\circ \prime} \Delta_{1+R_{\circ}} X_{t-1}-\mathcal{D}_{R} P_{t-2}-\mathcal{I}_{R} \Delta_{1} D_{t-1} .
$$

Due to (B.14) then $P_{t-2}$ is the sum of $R_{\circ}^{-1} W_{t-1}^{\circ}$ and some linear combination of $U_{t-1}^{\circ}, V_{t-1}^{\circ}$, while $\Delta_{1} D_{t-1}$ is some other linear combination of $U_{t-1}^{\circ}, V_{t-1}^{\circ}$.

The second coordinate of $U_{t-1}$ is $\beta_{\rho}^{\prime} \Delta_{1} X_{t-1}=\Delta_{1} D_{t-1}$ and does not depend on $R$.

The remaining coordinates of $U_{t-1}$ are of the type $\Delta_{1} \Delta_{1+R} X_{t-j}$. These are rewritten using (B.15). Thus, pre-multiplying $\Delta_{1} X_{t-1}$ by $I_{p}=\bar{\beta}_{\rho \perp}^{\circ} \beta_{\rho \perp}^{\circ \prime}+\bar{\beta}_{\rho}^{\circ} \beta_{\rho}^{\circ \prime}$ it is seen that $\Delta_{1} \Delta_{1+R} X_{t-j}$ is the sum of $\Delta_{1} \Delta_{1+R_{\circ}} X_{t-j}-\mathcal{D}_{R}\left(1+R_{\circ}\right)^{-j} \bar{\beta}_{\rho \perp}^{\circ} \beta_{\rho \perp}^{\circ \prime} \Delta_{1} X_{t-1}$ and some linear combination of $U_{t-1}^{\circ}, V_{t-1}^{\circ}$.

Product sums. The first component of interest is

$$
\begin{aligned}
\frac{1}{T} \sum_{t=1}^{T} \varepsilon_{D \cdot M, t}^{\circ} \mathcal{R}_{t}^{\prime} \stackrel{a . s}{=} \frac{1}{T} \sum_{t=1}^{T} \varepsilon_{D \cdot M, t}^{\circ} \mathcal{R}_{t}^{\circ \prime}-\mathcal{D}_{R} \frac{1}{T} \sum_{t=1}^{T} \varepsilon_{D \cdot M, t}^{\circ} W_{t-1}^{\circ} \mathcal{H}^{\prime} \\
-\frac{1}{T} \sum_{t=1}^{T} \varepsilon_{D \cdot M, t}^{\circ}\left(U_{t-1}^{\circ \prime}, V_{t-1}^{\circ}\right)\left(\mathcal{D}_{R} \mathcal{H}_{2}^{\prime}+\mathcal{I}_{R} \mathcal{H}_{3} e_{2}^{\prime}\right) .
\end{aligned}
$$

The processes $U_{t-1}^{\circ}, V_{t-1}^{\circ}$ are of polynomial order, see Nielsen (2005, Theorem 5.1), so $T^{-1} \sum_{t=1}^{T} \varepsilon_{D \cdot M, t}^{\circ}\left(U_{t-1}^{\circ \prime}, V_{t-1}^{\circ}\right)^{\prime}=\mathrm{o}_{\text {pol }}$ a.s. Note that the first coordinate $\frac{1}{T} \sum_{t=1}^{T} \varepsilon_{D \cdot M, t}^{\circ} M_{t}$ does not have an $\mathcal{I}_{R}$ component.

By a similar argument then, with $S_{1}=T^{-1} \sum_{t=1}^{T} \mathcal{R}_{t}^{\circ}\left(U_{t-1}^{\circ \prime}, V_{t-1}^{\circ}\right)^{\prime}\left(\mathcal{D}_{R} \mathcal{H}_{2}^{\prime}+\mathcal{I}_{R} \mathcal{H}_{3} e_{2}^{\prime}\right)$,

$$
\frac{1}{T} \sum_{t=1}^{T} \mathcal{R}_{t}^{\circ} \mathcal{R}_{t}^{\prime}=\frac{1}{T} \sum_{t=1}^{T} \mathcal{R}_{t}^{\circ} \mathcal{R}_{t}^{\circ \prime}-\mathcal{D}_{R} \frac{1}{T} \sum_{t=1}^{T} \mathcal{R}_{t}^{\circ} W_{t-1}^{\circ} \mathcal{H}^{\circ \prime}-S_{1},
$$

The processes $\mathcal{R}_{t}^{\circ}, U_{t-1}^{\circ}, V_{t-1}^{\circ}$ are of polynomial order, see Nielsen (2005, Theorem 5.1) so $\sum_{t=1}^{T} \mathcal{R}_{t}^{\circ}\left(U_{t-1}^{\circ \prime}, V_{t-1}^{\circ}\right)^{\prime}=\mathrm{o}_{\text {pol }}$ a.s.

By a similar argument then, with $S_{1}$ as above and

$$
\begin{aligned}
S_{2} & =\mathcal{D}_{R} \mathcal{H}^{\circ} \frac{1}{T} \sum_{t=1}^{T} W_{t-1}^{\circ}\left(U_{t-1}^{\circ \prime}, V_{t-1}^{\circ}\right)\left(\mathcal{D}_{R} \mathcal{H}_{2}^{\prime}+\mathcal{I}_{R} \mathcal{H}_{3} e_{2}^{\prime}\right), \\
S_{3} & =\left(\mathcal{D}_{R} \mathcal{H}_{2}+\mathcal{I}_{R} e_{2} \mathcal{H}_{3}^{\prime}\right) \frac{1}{T} \sum_{t=1}^{T}\left(U_{t-1}^{\circ \prime}, V_{t-1}^{\circ}\right)\left(U_{t-1}^{\circ \prime}, V_{t-1}^{\circ}\right)\left(\mathcal{D}_{R} \mathcal{H}_{2}^{\prime}+\mathcal{I}_{R} \mathcal{H}_{3} e_{2}^{\prime}\right),
\end{aligned}
$$

it holds that

$$
\begin{aligned}
\frac{1}{T} \sum_{t=1}^{T} \mathcal{R}_{t} \mathcal{R}_{t}^{\prime}= & \frac{1}{T} \sum_{t=1}^{T} \mathcal{R}_{t}^{\circ} \mathcal{R}_{t}^{\circ \prime}-\mathcal{D}_{R} \frac{1}{T} \sum_{t=1}^{T}\left(\mathcal{R}_{t}^{\circ} W_{t-1}^{\circ} \mathcal{H}^{\circ \prime}+\mathcal{H}^{\circ} W_{t-1}^{\circ} \mathcal{R}_{t}^{\circ}\right)^{\prime} \\
& +\mathcal{D}_{R}^{2} \frac{1}{T} \sum_{t=1}^{T} \mathcal{H}^{\circ}\left(W_{t-1}^{\circ}\right)^{2} \mathcal{H}^{\circ \prime}-S_{1}-S_{1}^{\prime}+S_{2}+S_{2}^{\prime}+S_{3} .
\end{aligned}
$$

As argued above $\sum_{t=1}^{T} \mathcal{R}_{t}^{\circ}\left(U_{t-1}^{\circ \prime}, V_{t-1}^{\circ}\right)^{\prime}$ and $\sum_{t=1}^{T}\left(U_{t-1}^{\circ \prime}, V_{t-1}^{\circ}\right)\left(U_{t-1}^{\circ \prime}, V_{t-1}^{\circ}\right)$ are of polynomial order while $T^{-1 / 2} \sum_{t=1}^{T} \mathcal{R}_{t}^{\circ} W_{t-1}^{\circ}=\mathrm{o}\left(\rho_{\circ}^{T}\right)$ a.s. by Lemma B.1 $(i, v, v i)$. 


\section{B.3 Consistency under $\mathrm{H}_{B}$}

The expression for the martingale difference $M_{t}$ is quadratic in the unknown parameters, so global consistency can be proved under $\mathrm{H}_{B}$ in contrast to the co-explosive analysis in Nielsen (2010). The starting point is the profile log likelihood for the parameter $R$. Let $\hat{\sigma}_{M}^{2}(R)$ and $\hat{\sigma}_{D \cdot M}^{2}(R)$ denote the residual variances of the regression equations (3.6), (3.7). Then the profile log likelihood is

$$
\ell(R)=-\frac{T}{2} \log \left\{\hat{\sigma}_{M}^{2}(R) \hat{\sigma}_{D \cdot M}^{2}(R)\right\} .
$$

The residual variances at $R_{\circ}$ satisfy the following results.

Lemma B.3 Assume $A, B, C$. Then

(i) $\hat{\sigma}_{M}^{2}\left(R_{\circ}\right)=T^{-1} \sum_{t=1}^{T} M_{t}^{\circ 2}=S_{\varepsilon_{M} \varepsilon_{M}}^{\circ} \stackrel{a . s .}{\rightarrow} \sigma_{M M}^{\circ}$,

(ii) $\hat{\sigma}_{D \cdot M}^{2}\left(R_{\circ}\right) \stackrel{\text { a.s. }}{\rightarrow} \sigma_{D D \cdot M}^{\circ}$.

(iii) $2 \ell\left(R_{\circ}\right)=-T \log \operatorname{det}\left(S_{\varepsilon \varepsilon}^{\circ}\right)+\sigma_{D D \cdot M}^{-1} \hat{\Sigma}_{\varepsilon_{D \cdot M} U} \Sigma_{U U}^{\circ-1} \hat{\Sigma}_{U \varepsilon_{D \cdot M}}+\mathrm{o}(1)$.

Proof of Lemma B.3. (i) Use $M_{t}^{\circ}=\varepsilon_{M, t}^{\circ}$.

(ii) Note $\hat{\sigma}_{D \cdot M}^{2}\left(R_{\circ}\right)=T^{-1} \sum_{t=1}^{T}\left(\varepsilon_{D \cdot M, t}^{\circ} \mid \mathcal{R}_{t}^{\circ}\right)^{2}$. Since $\mathcal{R}_{t}=\left(\varepsilon_{M, t}^{\circ}, U_{t-1}^{\circ}\right)^{\prime}$, see (B.6), and $\varepsilon_{D \cdot M, t}^{\circ}, \varepsilon_{M, t}^{\circ}, U_{t-1}^{\circ}$ are asymptotically uncorrelated then Lemma B.1 $(i)$ implies

$\hat{\sigma}_{D \cdot M}^{2}\left(R_{\circ}\right) \stackrel{\text { a.s. }}{=} S_{\varepsilon_{D \cdot M} \varepsilon_{D \cdot M}}^{\circ}-\left\{S_{\varepsilon_{D \cdot M} \varepsilon_{M}}^{\circ} S_{\varepsilon_{M} \varepsilon_{M}}^{\circ-1} S_{\varepsilon_{M} \varepsilon_{D \cdot M}}^{\circ}+S_{\varepsilon_{D} \cdot M}^{\circ} S_{U U}^{\circ-1} S_{U \varepsilon_{D} \cdot M}^{\circ}\right\}\left\{1+\mathrm{o}\left(T^{-1 / 4}\right)\right\}$.

Lemma B.1(ii,vii) shows $S_{\varepsilon_{D \cdot M} \varepsilon_{M}}^{\circ}, S_{\varepsilon_{D \cdot M} U}^{\circ}=\mathrm{o}\left(T^{-3 / 8}\right)$ while $S_{\varepsilon_{M} \varepsilon_{M}}^{\circ-1}, S_{U U}^{\circ-1}$ converge so

$$
\hat{\sigma}_{D \cdot M}^{2}\left(R_{\circ}\right) \stackrel{a . s .}{=} S_{\varepsilon_{D \cdot M} \varepsilon_{D} \cdot M}^{\circ}-S_{\varepsilon_{D \cdot M} \varepsilon_{M}}^{\circ} S_{\varepsilon_{M} \varepsilon_{M}}^{\circ-1} S_{\varepsilon_{M} \varepsilon_{D \cdot M}}^{\circ}-S_{\varepsilon_{D} \cdot M}^{\circ} S_{U U}^{\circ-1} S_{U \varepsilon_{D} \cdot M}^{\circ}+\mathrm{o}\left(T^{-1}\right),
$$

and in particular $\hat{\sigma}_{D \cdot M}^{2}\left(R_{\circ}\right) \rightarrow \sigma_{D D \cdot M}^{\circ}$ as desired.

(iii) Apply the expansion $\log (1+h)=h+\mathrm{O}\left(h^{2}\right)$ to (B.17) keeping the first two terms as the main term and noting $S_{\varepsilon_{D \cdot M} \varepsilon_{M}}^{\circ}, S_{\varepsilon_{D \cdot M} U}^{\circ}=\mathrm{o}\left(T^{-3 / 8}\right)$ to get

$$
\begin{aligned}
-T \log \left\{\hat{\sigma}_{D \cdot M}^{2}\left(R_{\circ}\right)\right\} \stackrel{\text { a.s. }}{=}-T \log \left(S_{\varepsilon_{D \cdot M} \varepsilon_{D \cdot M}}^{\circ}\right. & \left.-S_{\varepsilon_{D \cdot M} \varepsilon_{M}}^{\circ} S_{\varepsilon_{M} \varepsilon_{M}}^{\circ-1} S_{\varepsilon_{M} \varepsilon_{D} \cdot M}^{\circ}\right) \\
& +T S_{\varepsilon_{D \cdot M}^{\circ}-1}^{\circ \varepsilon_{D} M} S_{\varepsilon_{D \cdot M} U}^{\circ} S_{U U}^{\circ-1} S_{U \varepsilon_{D \cdot M}}^{\circ}+\mathrm{o}(1) .
\end{aligned}
$$

Insert this and $-T \log \left\{\hat{\sigma}_{M}^{2}\left(R_{\circ}\right)\right\}=-T \log S_{\varepsilon_{M} \varepsilon_{M}}^{\circ}$ into (B.16) to get

$2 \ell\left(R_{\circ}\right)=-T \log \left(S_{\varepsilon_{M} \varepsilon_{M}}^{\circ} S_{\varepsilon_{D} \cdot M}^{\circ \varepsilon_{D} \cdot M}-S_{\varepsilon_{D} \cdot M}^{\circ \varepsilon_{M}}\right)+T S_{\varepsilon_{D} \cdot M \varepsilon_{D} \cdot M}^{\circ-1} S_{\varepsilon_{D} \cdot M}^{\circ} S_{U U}^{\circ-1} S_{U \varepsilon_{D} \cdot M}^{\circ}+\mathrm{o}(1)$.

Due to the identity

$$
\operatorname{det}\left(S_{\varepsilon \varepsilon}^{\circ}\right)=\operatorname{det}\left\{\frac{1}{T} \sum_{t=1}^{T}\left(\begin{array}{c}
\varepsilon_{M, t}^{\circ} \\
\varepsilon_{D, t}^{\circ}
\end{array}\right)^{\otimes 2}\right\}=\operatorname{det}\left\{\frac{1}{T} \sum_{t=1}^{T}\left(\begin{array}{c}
\varepsilon_{D \cdot M, t}^{\circ} \\
\varepsilon_{D, t}^{\circ}
\end{array}\right)^{\otimes 2}\right\}
$$

the first term of $2 \ell\left(R_{\circ}\right)$ is $-T \log \operatorname{det}\left(S_{\varepsilon \varepsilon}^{\circ}\right)$. For the second term note $S_{U U}^{\circ} \rightarrow \Sigma_{U U}^{\circ}$ and $S_{\varepsilon_{D \cdot M} \varepsilon_{D \cdot M}}^{\circ} \rightarrow \sigma_{D D \cdot M}$ while $T^{1 / 2} S_{\varepsilon_{D \cdot M} U}^{\circ}=\hat{\Sigma}_{\varepsilon_{D \cdot M} U}$. 
Lemma B.4 Consider the maximum likelihood estimators under $\mathrm{H}_{B}$. Assume A, B, C. Then $\hat{R}-R=\mathrm{o}\left(T^{1 / 2} \rho^{-T}\right)$ a.s.

Proof of Lemma B.4. Let $R_{\circ}$ denote the true value of $R$.

Likelihood value at $R_{\circ}$. Lemma B.3 shows $\hat{\sigma}_{M}^{2}\left(R_{\circ}\right)=T^{-1} \sum_{t=1}^{T} \varepsilon_{M, t}^{\circ 2}$ and $\hat{\sigma}_{D \cdot M}^{2}\left(R_{\circ}\right)=$ $T^{-1} \sum_{t=1}^{T}\left(\varepsilon_{D \cdot M, t}^{\circ} \mid \mathcal{R}_{t}^{\circ}\right)^{2}$. Lemma B.1 $(i, v i)$ then implies

$$
\hat{\sigma}_{M}^{2}\left(R_{\circ}\right) \stackrel{a . s .}{\rightarrow} \sigma_{M}^{\circ 2}, \quad \hat{\sigma}_{D \cdot M}^{2}\left(R_{\circ}\right) \stackrel{a . s .}{\rightarrow} \sigma_{D \cdot M}^{\circ 2} \cdot
$$

Likelihood value outside neighbourhood of $R_{\circ}$. For any $\delta>0$ consider an $R$ so $\left|N_{W}^{-1}\left(R-R_{\circ}\right)\right|>\delta$. It is to be shown that

$$
\liminf \operatorname{in}_{T \rightarrow \infty} \hat{\sigma}_{M}^{2}\left(R_{\circ}\right) \stackrel{\text { a.s. }}{=} \sigma_{M}^{\circ 2}+\delta^{2} \kappa_{P}, \quad \liminf _{T \rightarrow \infty} \hat{\sigma}_{D \cdot M}^{2}\left(R_{\circ}\right) \stackrel{a . s .}{\geq} \sigma_{D \cdot M}^{2},
$$

where $\kappa_{P}=\mathcal{G}^{\circ 2} \lim _{T \rightarrow \infty} \rho_{\circ}^{-2 T} \sum_{t=1}^{T}\left(W_{t-1}^{\circ}\right)^{2}>0$ a.s. for $\rho_{\circ}=1+R_{\circ}$.

For the first result note that by (B.13) then

$$
\hat{\sigma}_{M}^{2}(R) \stackrel{\text { a.s. }}{=} S_{M M}^{\circ}+2\left(R_{\circ}-R\right) \mathcal{G}^{\circ} S_{M W}^{\circ}+\left(R_{\circ}-R\right)^{2} \mathcal{G}^{\circ 2} S_{W W}^{\circ}+\mathrm{O}\left(T^{-1}\right) .
$$

Due to Lemma B.1(i,iii,vi) then

$$
\hat{\sigma}_{M}^{2}(R) \stackrel{\text { a.s. }}{=} \sigma_{M}^{\circ 2}+T^{-1} \rho_{\circ}^{2 T}\left(R_{\circ}-R\right)^{2} \kappa_{P}+2 T^{-1 / 2} \rho_{\circ}^{T}\left(R_{\circ}-R\right) \mathrm{o}(1)+\mathrm{o}(1) .
$$

Thus, for any $R$ outside a neighbourhood of $R_{\circ}$ this has the stated limes inferior.

For the second result write the residual variance as

$$
\hat{\sigma}_{D \cdot M}^{2}(R)=\frac{1}{T} \sum_{t=1}^{T}\left(\Delta_{1} D_{t} \mid \mathcal{R}_{t}\right)^{2}
$$

As $M_{t}=M_{t}^{\circ}+\left(R_{\circ}-R\right) P_{t-1}$ the regressor $\mathcal{R}_{t}$ is a linear combination of $M_{t}^{\circ}, \mathcal{S}_{t-1}^{\circ}$. Moreover, $\Delta_{1} D_{t}=\theta_{\circ}^{\prime} \mathcal{R}_{t}^{\circ}+\varepsilon_{D \cdot M, t}^{\circ}$ for some $\theta_{\circ}$ while $\mathcal{R}_{t}^{\circ}$ is a linear combination of $M_{t}^{\circ}, \mathcal{S}_{t-1}^{\circ}$ and $M_{t}^{\circ}=\varepsilon_{M, t}^{\circ}$. Thus

$$
\hat{\sigma}_{D \cdot M}^{2}(R) \geq \frac{1}{T} \sum_{t=1}^{T}\left(\Delta_{1} D_{t} \mid M_{t}^{\circ}, \mathcal{S}_{t-1}^{\circ}\right)^{2}=\frac{1}{T} \sum_{t=1}^{T}\left(\varepsilon_{D \cdot M, t}^{\circ} \mid \varepsilon_{M, t}^{\circ}, \mathcal{S}_{t-1}^{\circ}\right)^{2} .
$$

Since the sample correlations of $\varepsilon_{D \cdot M, t}^{\circ}, \varepsilon_{M, t}^{\circ}$ and $\mathcal{S}_{t-1}^{\circ}$ vanish asymptotically then $\hat{\sigma}_{D \cdot M}^{2}(R)$ has the stated limes inferior.

Continuity of likelihood function. The profile log likelihood $\ell(R)$ is continuous and will, asymptotically, attain its minimum in a compact interval $\left|N_{W}^{-1}\left(R-R_{\circ}\right)\right| \leq \delta$ as it is large outside the interval. This shows the desired consistency. 


\section{B.4 Expanding likelihood under $\mathrm{H}_{B}$}

The profile likelihood for $R$ is analysed. The first Lemma expands log determinants.

Lemma B.5 $\log \operatorname{det}(I+h)=\operatorname{tr}(h)-\frac{1}{2} \operatorname{tr}\left(h^{2}\right)+\frac{1}{3} \operatorname{tr}\left(h^{3}\right)+\mathrm{O}\left(\|h\|^{4}\right)$.

Proof of Lemma B.5. The matrix $h$ can be decomposed as $h=A J A^{-1}$ where $J$ is a triangular, Jordan matrix with diagonal elements $\lambda_{j}$. Thus, $I+h=A(I+J) A^{-1}$ and $\operatorname{det}(I+h)=\operatorname{det}(I+J)=\prod_{j=1}^{\operatorname{dim} h}\left(1+\lambda_{j}\right)$. By the expansion $\log (1+x)=$ $x-x^{2} / 2+x^{3} / 6+\mathrm{O}\left(x^{4}\right)$ it holds

$$
\log \operatorname{det}(I+J)=\sum_{j=1}^{\operatorname{dim} h} \log \left(1+\lambda_{j}\right)=\sum_{j=1}^{\operatorname{dim} h}\left\{\lambda_{j}-\frac{1}{2} \lambda_{j}^{2}+\frac{1}{3} \lambda_{j}^{2}+\mathrm{O}\left(\lambda_{j}^{4}\right)\right\} .
$$

Noting that $\operatorname{tr}\left(J^{k}\right)=\sum_{j=1}^{\operatorname{dim} h} \lambda_{j}^{k}$ and $\operatorname{tr}\left(A^{k}\right)=\operatorname{tr}\left(J^{k}\right)$ the desired result follows.

The next step is to write the profile likelihood in terms quadratic functions in $R$.

Lemma B.6 Assume A, B, C. Under $\mathrm{H}_{B}$ the profile likelihood has expansion

$$
2\left\{\ell(R)-\ell\left(R_{\circ}\right)\right\} \stackrel{a . s .}{=} 2\left\{\tilde{\ell}(R)-\tilde{\ell}\left(R_{\circ}\right)\right\}+\mathrm{o}(1)
$$

for $\left|R-R_{\circ}\right| \leq c T^{1 / 2} \rho_{\circ}^{-T}$ for any $c>0$. Here $\tilde{\ell}(R)=\tilde{\ell}_{M}(R)+\tilde{\ell}_{\mathcal{R} \cdot \Delta}(R)-\tilde{\ell}_{\mathcal{R}}(R)$ with

$$
\begin{aligned}
\tilde{\ell}_{M}(R) & =-\frac{T}{2} \log \left(S_{\varepsilon_{M} \varepsilon_{M}}^{\circ}-2 \mathcal{D}_{R} \mathcal{G}^{\circ} S_{\varepsilon_{M} W}^{\circ}+\mathcal{D}_{R}^{2} \mathcal{G}^{\circ 2} S_{W W}^{\circ}\right), \\
\tilde{\ell}_{\mathcal{R} \cdot \Delta}(R) & =-\frac{T}{2} \log \operatorname{det}\left\{S_{\mathcal{R} \mathcal{R} \cdot \Delta}^{\circ}-\mathcal{D}_{R}\left(S_{\mathcal{R} W \cdot \Delta}^{\circ} \mathcal{H}^{\circ \prime}+\mathcal{H}^{\circ} S_{W \mathcal{R} \cdot \Delta}^{\circ}\right)+\mathcal{D}_{R}^{2} \mathcal{H}^{\circ} S_{W W \cdot \Delta}^{\circ} \mathcal{H}^{\circ \prime}\right\}, \\
\tilde{\ell}_{\mathcal{R}}(R) & =-\frac{T}{2} \log \operatorname{det}\left\{S_{\mathcal{R} \mathcal{R}}^{\circ}-\mathcal{D}_{R}\left(S_{\mathcal{R} W}^{\circ} \mathcal{H}^{\circ \prime}+\mathcal{H}^{\circ} S_{W \mathcal{R}}^{\circ}\right)+\mathcal{D}_{R}^{2} \mathcal{H}^{\circ} S_{W W}^{\circ} \mathcal{H}^{\circ \prime}\right\} .
\end{aligned}
$$

Proof of Lemma B.6. Profile likelihood. This is given by

$$
2 \ell(R)=-T \log \left\{\hat{\sigma}_{M}^{2}(R)\right\}-T \log \left\{\hat{\sigma}_{D \cdot M}^{2}(R)\right\} .
$$

It will be shown that this is quadratic in $R$ up to an approximation.

Component involving $\hat{\sigma}_{M}^{2}(R)$. Since $\hat{\sigma}_{M}^{2}(R)=T^{-1} \sum_{t=1}^{T} M_{t}^{2}$ consider the expansion (B.13). Since $\mathcal{D}_{R}=\mathrm{o}\left(T^{1 / 2} \rho_{\circ}^{-T}\right)$ then

$$
\hat{\sigma}_{M}^{2}(R) \stackrel{\text { a.s. }}{=} S_{\varepsilon_{M} \varepsilon_{M}}^{\circ}-2 \mathcal{D}_{R} \mathcal{G}^{\circ} S_{\varepsilon_{M} W}^{\circ}+\mathcal{D}_{R}^{2} \mathcal{G}^{\circ 2} S_{W W}^{\circ}+\mathrm{o}\left(T^{-1}\right) .
$$

The expansion $\log (1+h)=\mathrm{O}(h)$ shows

$$
\log \left\{\hat{\sigma}_{M}^{2}(R)\right\} \stackrel{\text { a.s. }}{=} \log \left(S_{\varepsilon_{M} \varepsilon_{M}}^{\circ}-2 \mathcal{D}_{R} \mathcal{G}^{\circ} S_{\varepsilon_{M} W}^{\circ}+\mathcal{D}_{R}^{2} \mathcal{G}^{\circ 2} S_{W W}^{\circ}\right)+\mathrm{o}\left(T^{-1}\right) .
$$


Component involving $\hat{\sigma}_{D \cdot M}^{2}(R)$. First, use partitioned inversion to get

$$
\begin{aligned}
\log \left\{\hat{\sigma}_{D \cdot M}^{2}(R)\right\}= & \log \operatorname{det}\left\{T^{-1} \sum_{t=1}^{T}\left(\mathcal{R}_{t} \mid \Delta_{1} D_{t}\right)^{\otimes 2}\right\} \\
& -\log \operatorname{det}\left\{T^{-1} \sum_{t=1}^{T}\left(\mathcal{R}_{t}\right)^{\otimes 2}\right\}+\log \left\{T^{-1} \sum_{t=1}^{T}\left(\Delta_{1} D_{t}\right)^{2}\right\} .
\end{aligned}
$$

The last term does not depend on $R$.

For the second term of (B.21) apply Lemma B.2 to get

$$
\frac{1}{T} \sum_{t=1}^{T} \mathcal{R}_{t} \mathcal{R}_{t}^{\prime \prime} \stackrel{\text { a.s. }}{=} S_{\mathcal{R} \mathcal{R}}^{\circ}-\mathcal{D}_{R}\left(S_{\mathcal{R} W}^{\circ} \mathcal{H}^{\circ \prime}+\mathcal{H} S_{W \mathcal{R}}^{\circ}\right)+\mathcal{D}_{R}^{2} \mathcal{H}^{\circ} S_{W W}^{\circ} \mathcal{H}^{\circ \prime}+\mathrm{o}\left(T^{-1}\right) .
$$

Applying the log determinant expansion in (B.5) it follows that

$$
\log \operatorname{det}\left\{T^{-1} \sum_{t=1}^{T}\left(\mathcal{R}_{t}\right)^{\otimes 2}\right\} \stackrel{\text { a.s. }}{=} \tilde{\ell}_{R}(R)+\mathrm{o}\left(T^{-1}\right) .
$$

Apply a similar argument for the first term of (B.21) to get

$$
\log \operatorname{det}\left\{T^{-1} \sum_{t=1}^{T}\left(\mathcal{R}_{t} \mid \Delta_{1} D_{t}\right)^{\otimes 2}\right\} \stackrel{\text { a.s. }}{=} \tilde{\ell}_{R \cdot \Delta}(R)+\mathrm{o}\left(T^{-1}\right) .
$$

Profile likelihood expansion. Insert (B.22) and (B.23) into the expression for $\log \left(\hat{\sigma}_{D \cdot M}^{2}\right)$ in (B.21) and in turn insert this and the expression (B.20) for $\log \left(\hat{\sigma}_{M}^{2}\right)$ into the profile likelihood (B.19) to get

$$
2 \ell(R) \stackrel{\text { a.s. }}{=} 2 \tilde{\ell}(R)-T \log \left(S_{\Delta \Delta}\right)+\mathrm{o}(1) .
$$

Finally note that $\ell_{R}\left(R_{\circ}\right)=\tilde{\ell}_{R}\left(R_{\circ}\right)-T \log \left(S_{\Delta \Delta}\right)$.

The derivatives of the approximation $\tilde{\ell}$ to the profile likelihood are considered.

Lemma B.7 Assume A, B, C. Under $\mathrm{H}_{B}$ then

$$
\begin{aligned}
& \tilde{\ell}^{\prime}\left(R_{\circ}\right)=\rho_{\circ}^{T}\left\{\mathcal{G}^{\circ} S_{\varepsilon_{M} \varepsilon_{M}}^{\circ-1} \hat{\Sigma}_{\varepsilon_{M} W}+\mathcal{H}^{\circ \prime}\left(S_{\mathcal{R} R \cdot \Delta}^{\circ-1} \hat{\Sigma}_{\mathcal{R} W \cdot \Delta}-S_{\mathcal{R} R}^{\circ-1} \hat{\Sigma}_{\mathcal{R} W}\right)\right\}, \\
& \tilde{\ell}^{\prime \prime}\left(R_{\circ}\right) \stackrel{\text { a.s. }}{=}-\rho_{\circ}^{2 T} \Sigma_{W W}^{\circ}\left\{\mathcal{G}^{\circ 2} S_{\varepsilon_{M} \varepsilon_{M}}^{\circ-1}+\mathcal{H}^{\circ \prime}\left(S_{\mathcal{R R} \cdot \Delta}^{\circ-1}-S_{\mathcal{R R}}^{\circ-1}\right) \mathcal{H}^{\circ}\right\}\{1+\mathrm{o}(1)\},
\end{aligned}
$$

where $\hat{\Sigma}_{\mathcal{R} W}=\sum_{t=1}^{T} \mathcal{R}_{t}^{\circ} W_{t-1}^{\circ}$. It holds that

$$
\tilde{\ell}^{\prime}\left(R_{\circ}\right) \stackrel{a . s .}{=} \mathrm{o}\left(T^{1 / 4} \rho_{\circ}^{T}\right), \quad\left\{\tilde{\ell}^{\prime \prime}\left(R_{\circ}\right)\right\}^{-1}=\mathrm{O}\left(\rho_{\circ}^{-2 T}\right), \quad \tilde{\ell}^{\prime \prime \prime}\left(R_{\circ}\right)=\mathrm{o}\left(T^{-3 / 4} \rho_{\circ}^{3 T}\right) .
$$


Proof of Lemma B.7. Term $\tilde{\ell}_{M}(R)$. This satisfies

$$
-(2 / T) \tilde{\ell}_{M}(R)=\log \left(S_{\varepsilon_{M} \varepsilon_{M}}^{\circ}\right)+\log (1+h) .
$$

where $h=-2 \mathcal{D}_{R} \mathcal{G}^{\circ} S_{\varepsilon_{M} \varepsilon_{M}}^{\circ-1} S_{\varepsilon_{M} W}^{\circ}+\mathcal{D}_{R}^{2} \mathcal{G}^{\circ 2} S_{\varepsilon_{M} \varepsilon_{M}}^{\circ-1} S_{W W}^{\circ}$. Apply the log expansion in Lemma B.5. Rearrange to get an expansion in $\mathcal{D}_{R}$ which is

$$
\begin{aligned}
\log (1+h)= & -2 \mathcal{D}_{R} \mathcal{G}^{\circ} S_{\varepsilon_{M} \varepsilon_{M}}^{\circ-1} S_{\varepsilon_{M} W}^{\circ}+\mathcal{D}_{R}^{2} \mathcal{G}^{\circ 2}\left(S_{\varepsilon_{M} \varepsilon_{M}}^{\circ-1} S_{W W}-2 S_{\varepsilon_{M} \varepsilon_{M}}^{\circ-2} S_{\varepsilon_{M} W}^{\circ 2}\right) \\
& +\mathcal{D}_{R}^{3} \mathcal{G}^{\circ 3}\left(2 S_{\varepsilon_{M} \varepsilon_{M}}^{\circ-2} S_{\varepsilon_{M} W}^{\circ} S_{W W}-\frac{8}{3} S_{\varepsilon_{M} \varepsilon_{M}}^{\circ-3} S_{\varepsilon_{M} W}^{\circ 3}\right)+\mathrm{O}\left(\mathcal{D}_{R}^{4}\right)
\end{aligned}
$$

Hence the coefficient to $\mathcal{D}_{R}$ gives the first derivative $\tilde{\ell}_{M}^{\prime}\left(R_{\circ}\right)=T \mathcal{G} S_{\varepsilon_{M} \varepsilon_{M}}^{\circ-1} S_{\varepsilon_{M} W}^{\circ}$. Replacing $T S_{\varepsilon_{M} W}^{\circ}=T^{1 / 2}\left(\rho_{\circ}^{-T} \sum_{t=1}^{T} \varepsilon_{M, t}^{\circ} W_{t-1}^{\circ}\right)\left(T^{-1 / 2} \rho_{\circ}^{T}\right)=T^{1 / 2} \hat{\Sigma}_{\varepsilon_{M} W} N_{W}^{-1}$ gives

$$
\tilde{\ell}_{M}^{\prime}\left(R_{\circ}\right)=T^{1 / 2} \mathcal{G} S_{\varepsilon_{M} \varepsilon_{M}}^{\circ-1} \hat{\Sigma}_{\varepsilon_{M} W} N_{W}^{-1} .
$$

Likewise the second and third derivates are

$$
\begin{aligned}
& \tilde{\ell}_{M}^{\prime \prime}\left(R_{\circ}\right)=(2 !)(-T / 2) \mathcal{G}^{\circ 2}\left(S_{\varepsilon_{M} \varepsilon_{M}}^{\circ-1} S_{W W}-2 S_{\varepsilon_{M} \varepsilon_{M}}^{\circ-2} S_{\varepsilon_{M} W}^{\circ 2}\right) \\
& \tilde{\ell}_{M}^{\prime \prime \prime}\left(R_{\circ}\right)=(3 !)(-T / 2) \mathcal{G}^{\circ 3}\left(2 S_{\varepsilon_{M} \varepsilon_{M}}^{\circ-2} S_{\varepsilon_{M} W}^{\circ} S_{W W}-\frac{8}{3} S_{\varepsilon_{M} \varepsilon_{M}}^{\circ-3} S_{\varepsilon_{M} W}^{\circ 3}\right)
\end{aligned}
$$

Noting that $S_{\varepsilon_{M} \varepsilon_{M}}^{\circ-1}, S_{W W} N_{W}^{2}=\hat{\Sigma}_{W W}$ are convergent while $S_{\varepsilon_{M} W}^{\circ} N_{W}=\mathrm{o}\left(T^{-1 / 4}\right)$ then

$$
\tilde{\ell}_{M}^{\prime \prime}\left(R_{\circ}\right)=-T \mathcal{G}^{\circ 2} S_{\varepsilon_{M} \varepsilon_{M}}^{\circ-1} \Sigma_{W W}^{\circ} N_{W}^{-2}\{1+\mathrm{o}(1)\}, \quad \tilde{\ell}_{M}^{\prime \prime \prime}\left(R_{\circ}\right) \stackrel{\text { a.s. }}{=} \mathrm{o}\left(T^{-3 / 4} \rho_{\circ}^{3 T}\right) .
$$

Term $\tilde{\ell}_{\mathcal{R}}(R)$. This satisfies

$$
-(2 / T) \tilde{\ell}_{\mathcal{R}}(R)=\log \operatorname{det}\left(S_{\mathcal{R} \mathcal{R}}\right)+\log \operatorname{det}\left(I_{\operatorname{dim} \mathcal{R}}+h\right)
$$

where $h=-\mathcal{D}_{R} S_{\mathcal{R} R}^{\circ-1}\left(S_{\mathcal{R} W}^{\circ} \mathcal{H}^{\circ \prime}+\mathcal{H}^{\circ} S_{W \mathcal{R}}^{\circ}\right)+\mathcal{D}_{R}^{2} S_{\mathcal{R} \mathcal{R}}^{\circ-1} \mathcal{H}^{\circ} S_{W W}^{\circ} \mathcal{H}^{\circ \prime}$. Apply the log expansion in Lemma B.5. Rearrange to get an expansion in $\mathcal{D}_{R}$ which is

$$
\begin{aligned}
\log \operatorname{det}(I+h)= & -2 \mathcal{D}_{R} \operatorname{tr}\left\{S_{\mathcal{R} \mathcal{R}}^{\circ-1} S_{\mathcal{R} W}^{\circ} \mathcal{H}^{\circ \prime}\right\}+\mathcal{D}_{R}^{2}\left\{\operatorname{tr}\left(S_{\mathcal{R} \mathcal{R}}^{\circ-1} \mathcal{H}^{\circ} S_{W W}^{\circ} \mathcal{H}^{\circ \prime}\right)-\operatorname{tr}\left(\mathcal{B}^{2}\right) / 2\right\} \\
& +\mathcal{D}_{R}^{3}\left(\mathcal{B} S_{\mathcal{R} \mathcal{R}}^{\circ-1} \mathcal{H}^{\circ} S_{W W}^{\circ} \mathcal{H}^{\circ \prime} / 2-\mathcal{B}^{3} / 3\right)+\mathrm{O}\left(\mathcal{D}_{R}^{4}\right),
\end{aligned}
$$

where $\mathcal{B}=S_{\mathcal{R R}}^{\circ-1}\left(S_{\mathcal{R} W}^{\circ} \mathcal{H}^{\circ \prime}+\mathcal{H}^{\circ} S_{W \mathcal{R}}^{\circ}\right)$. By considerations as above it is seen that $\mathcal{B}=$ $\mathrm{o}\left(T^{-3 / 4} \rho_{\circ}^{T}\right)$ and the derivatives satisfy

$$
\begin{aligned}
& \tilde{\ell}_{\mathcal{R}}^{\prime}\left(R_{\circ}\right)=T^{1 / 2} \operatorname{tr}\left\{\mathcal{H}^{\circ \prime} S_{\mathcal{R} \mathcal{R}}^{\circ-1} \hat{\Sigma}_{\mathcal{R} W}\right\} N_{W}^{-1}, \quad \tilde{\ell}_{M}^{\prime \prime \prime}\left(R_{\circ}\right) \stackrel{\text { a.s. }}{=} \mathrm{o}\left(T^{-3 / 4} \rho_{\circ}^{3 T}\right), \\
& \tilde{\ell}_{M}^{\prime \prime}\left(R_{\circ}\right) \stackrel{\text { a.s. }}{=}-T \operatorname{tr}\left(\mathcal{H}^{\circ \prime} S_{\mathcal{R} R}^{\circ-1} \mathcal{H}^{\circ}\right) \Sigma_{W W}^{\circ} N_{W}^{-2}\{1+\mathrm{o}(1)\} .
\end{aligned}
$$

Term $\tilde{\ell}_{\mathcal{R} \cdot \Delta}(R)$. Same derivation as for $\tilde{\ell}_{\mathcal{R}}(R)$ replacing $S_{\mathcal{R} \mathcal{R}}, S_{\mathcal{R} W}$ and $S_{W W}$ by $S_{\mathcal{R} \cdot \Delta}, S_{\mathcal{R} W \cdot \Delta}$ and $S_{W W \cdot \Delta}=S_{W W}\{1+\mathrm{o}(1)\}$.

The expressions for the $\tilde{\ell}^{\prime}$ and $\tilde{\ell}^{\prime \prime}$ are simplified using the parameter $\tau_{\perp}^{\circ}$ from (B.10). 
Lemma B.8 Assume A, B, C. Under $\mathrm{H}_{B}$ then

$$
\begin{array}{rcl}
\tilde{\ell}^{\prime}\left(R_{\circ}\right) \stackrel{\text { a.s. }}{=} & \rho_{\circ}^{T}\left\{\tau_{\perp}^{\circ} \Omega_{\circ}^{-1} \hat{\Sigma}_{\varepsilon W}+\mathrm{o}\left(T^{-1 / 4}\right)\right\} \\
-\tilde{\ell}^{\prime \prime}\left(R_{\circ}\right) & \stackrel{\text { a.s. }}{=} & \rho_{\circ}^{2 T} \tau_{\perp}^{\circ} \Omega_{\circ}^{-1} \tau_{\perp}^{\circ} \Sigma_{W W}^{\circ}\{1+\mathrm{o}(1)\} .
\end{array}
$$

Proof of Lemma B.8. Product moment matrices. Recall from (B.7) that $\Delta_{1} D_{t}=\left(\omega^{\circ}, \theta^{\circ \prime}\right) \mathcal{R}_{t}^{\circ}+\varepsilon_{D \cdot M, t}^{\circ}$ and note $\omega^{\circ}=\sigma_{M M}^{\circ-1} \sigma_{D M}^{\circ}$. It holds, for all $\eta>0$, see Lemma B.1 $(i, v i i)$,

$$
\begin{aligned}
& S_{\mathcal{R} \mathcal{R}}^{\circ} \stackrel{a . s .}{\rightarrow}\left(\begin{array}{cc}
\sigma_{M M}^{\circ} & 0 \\
0 & \Sigma_{U U}^{\circ}
\end{array}\right), \\
& S_{\mathcal{R} \Delta}^{\circ} \stackrel{\text { a.s. }}{=}\left(\begin{array}{c}
\sigma_{M M}^{\circ} \omega^{\circ} \\
\Sigma_{U U}^{\circ} \theta^{\circ}
\end{array}\right)=\left(\begin{array}{c}
\sigma_{D M}^{\circ} \\
\Sigma_{U U}^{\circ} \theta^{\circ}
\end{array}\right)+\mathrm{o}\left(T^{\eta-1 / 2}\right) \text {. }
\end{aligned}
$$

Since $\Delta_{1} D_{t}$ also satisfies $\Delta_{1} D_{t}=\theta^{\circ} \mathcal{R}_{t}^{\circ}+\varepsilon_{D, t}^{\circ}$ then

$$
S_{\Delta \Delta} \stackrel{\text { a.s. }}{=} \sigma_{D D}^{\circ}+\theta^{\circ} \Sigma_{U U}^{\circ} \theta^{\circ}+\mathrm{o}\left(T^{\eta-1 / 2}\right) .
$$

Moreover, exploiting $\Delta_{1} D_{t}=\left(\omega^{\circ}, \theta^{\circ \prime}\right) \mathcal{R}_{t}^{\circ}+\varepsilon_{D \cdot M, t}^{\circ}$ and $\varepsilon_{D, t}^{\circ}=\varepsilon_{D \cdot M, t}^{\circ}+\omega^{\circ} \varepsilon_{M, t}^{\circ}$ it holds

$$
T S_{\mathcal{R} W}=\sum_{t=1}^{T}\left(\begin{array}{c}
\varepsilon_{M, t}^{\circ} \\
U_{t-1}^{\circ}
\end{array}\right) W_{t-1}^{\circ}, \quad T S_{\Delta W}=\hat{\Sigma}_{\Delta W}=\left(1, \theta^{\prime}\right) \sum_{t=1}^{T}\left(\begin{array}{c}
\varepsilon_{D, t}^{\circ} \\
U_{t-1}^{\circ}
\end{array}\right) W_{t-1}^{\circ} .
$$

Information. Combine the expressions (B.24), (B.25), (B.26) to get

$$
S_{\mathcal{R} R \cdot \Delta}^{\circ}=S_{\mathcal{R} \mathcal{R}}^{\circ}-S_{\mathcal{R} \Delta}^{\circ} S_{\Delta \Delta}^{-1} S_{\Delta \mathcal{R}}^{\circ} \stackrel{a . s .}{\rightarrow}\left(\begin{array}{cc}
\sigma_{M M}^{\circ} & 0 \\
0 & \Sigma_{U U}^{\circ}
\end{array}\right)-\left(\begin{array}{c}
\sigma_{D M}^{\circ} \\
\Sigma_{U U}^{\circ} \theta^{\circ}
\end{array}\right)^{\otimes 2} \frac{1}{\sigma_{D D}^{\circ}+\theta^{\circ} \Sigma_{U U}^{\circ} \theta^{\circ}} .
$$

The partitioned inversion formula $A_{11 \cdot 2}^{-1}=A_{11}^{-1}+A_{11}^{-1} A_{12} A_{22 \cdot 1}^{-1} A_{21} A_{11}^{-1}$ shows, noting that $\omega^{\circ}=\sigma_{M M}^{\circ-1} \sigma_{D M}^{\circ}$

$$
\begin{aligned}
\stackrel{S_{\mathcal{R} R \cdot \Delta}^{\circ}-S_{\mathcal{R} R}^{\circ-1}}{=} \frac{\left(\begin{array}{cc}
\sigma_{M M}^{\circ-1} & 0 \\
0 & \Sigma_{U Y}^{\circ-1}
\end{array}\right)\left(\begin{array}{c}
\sigma_{D M}^{\circ} \\
\Sigma_{U U}^{\circ} \theta^{\circ}
\end{array}\right)\left(\sigma_{D M}^{\circ}, \theta^{\circ \prime} \Sigma_{U U}^{\circ}\right)\left(\begin{array}{cc}
\sigma_{M M}^{\circ-1} & 0 \\
0 & \Sigma_{U U}^{\circ-1}
\end{array}\right)}{\sigma_{D D}^{\circ}+\theta^{\circ \prime} \Sigma_{U U}^{\circ} \theta^{\circ}-\left(\sigma_{D M}^{\circ}, \theta^{\circ \prime} \Sigma_{U U}^{\circ}\right)\left(\begin{array}{cc}
\sigma_{M M}^{\circ-1} & 0 \\
0 & \Sigma_{U U}^{\circ-1}
\end{array}\right)\left(\begin{array}{c}
\sigma_{D M}^{\circ} \\
\Sigma_{U U}^{\circ} \theta^{\circ}
\end{array}\right)}+\mathrm{o}\left(T^{-1 / 4}\right) \\
=\frac{1}{\sigma_{D D \cdot M}^{\circ}}\left(\begin{array}{c}
\omega^{\circ} \\
\theta^{\circ}
\end{array}\right)^{\otimes 2}+\mathrm{o}\left(T^{-1 / 4}\right) .
\end{aligned}
$$

Further, note that $S_{\varepsilon_{M} \varepsilon_{M}}^{\circ} \stackrel{\text { a.s. }}{\rightarrow} \sigma_{M M}^{\circ}$ while the definition of $\tau_{\perp}^{\circ}$ in (B.10) implies

$$
\left(\omega^{\circ}, \theta^{\circ}\right) \mathcal{H}=\left(\omega^{\circ},-1\right) \tau_{\perp}^{\circ}, \quad \mathcal{G}^{\circ}=(1,0) \tau_{\perp}^{\circ} .
$$


Combining these expressions shows

$$
\mathcal{G}^{\circ 2} S_{\varepsilon_{M} \varepsilon_{M}}^{\circ-1}+\mathcal{H}^{\prime}\left(S_{\mathcal{R} \mathcal{R} \cdot \Delta}^{-1}-S_{\mathcal{R} \mathcal{R}}^{-1}\right) \mathcal{H} \stackrel{\text { a.s. }}{\rightarrow} \tau_{\perp}^{\circ \prime}\left\{\sigma_{M M}^{-1}\left(\begin{array}{c}
1 \\
0
\end{array}\right)^{\otimes 2}+\sigma_{D D \cdot M}^{-1}\left(\begin{array}{c}
\omega^{\circ} \\
-1
\end{array}\right)^{\otimes 2}\right\} \tau_{\perp}^{\circ} .
$$

Finally, the desired expression follows since by partitioned inversion

$$
\Omega_{\circ}^{-1}=\sigma_{M M}^{-1}\left(\begin{array}{l}
1 \\
0
\end{array}\right)^{\otimes 2}+\sigma_{D D \cdot M}^{-1}\left(\begin{array}{c}
\omega^{\circ} \\
-1
\end{array}\right)^{\otimes 2}
$$

Score. Combine (B.25), (B.26), (B.27) to get

$$
\begin{aligned}
\hat{\Sigma}_{\mathcal{R} W \cdot \Delta}= & \hat{\Sigma}_{\mathcal{R} W}-S_{\mathcal{R} \Delta}^{\circ} S_{\Delta \Delta}^{-1} \hat{\Sigma}_{\Delta W} \\
& \stackrel{a . s .}{=} \hat{\Sigma}_{\mathcal{R} W}-\frac{1+\mathrm{o}\left(T^{\eta-1 / 2}\right)}{\sigma_{D D}^{\circ}+\theta^{\circ \prime} \Sigma_{U U}^{\circ} \theta^{\circ}}\left(\begin{array}{c}
\sigma_{D M}^{\circ} \\
\Sigma_{U U}^{\circ} \theta^{\circ}
\end{array}\right)\left(1, \theta^{\circ \prime}\right) \hat{\Sigma}_{\Delta W} .
\end{aligned}
$$

In a similar way write

$$
\begin{aligned}
& S_{\mathcal{R} \mathcal{R}}^{\circ-1} \hat{\Sigma}_{\mathcal{R} W}=S_{\mathcal{R R} \cdot \Delta}^{\circ-1} S_{\mathcal{R} \mathcal{R} \cdot \Delta}^{\circ} S_{\mathcal{R} \mathcal{R}}^{\circ-1} \hat{\Sigma}_{\mathcal{R} W} \\
& \stackrel{\text { a.s. }}{=} S_{\mathcal{R} \mathcal{R} \cdot \Delta}^{\circ-1}\left\{I_{2}-\left(\begin{array}{c}
\sigma_{D M}^{\circ} \\
\Sigma_{U U}^{\circ} \theta^{\circ}
\end{array}\right) \frac{1+\mathrm{o}\left(T^{\eta-1 / 2}\right)}{\sigma_{D D}^{\circ}+\theta^{\circ \prime} \Sigma_{U U}^{\circ} \theta^{\circ}}\right\}\left(\omega^{\circ}, \theta^{\circ \prime}\right) \hat{\Sigma}_{\mathcal{R} W} .
\end{aligned}
$$

These expression combine as

$$
\begin{aligned}
& S_{\mathcal{R} R \cdot \Delta}^{\circ-1} \hat{\Sigma}_{\mathcal{R} W \cdot \Delta}-S_{\mathcal{R} \mathcal{R}}^{\circ-1} \hat{\Sigma}_{\mathcal{R} W} \\
& \stackrel{\text { a.s. }}{=} S_{\mathcal{R} \mathcal{R} \cdot \Delta}^{\circ-1} \frac{\left\{1+\mathrm{o}\left(T^{\eta-1 / 2}\right)\right\}}{\sigma_{D D}^{\circ}+\theta^{\circ \prime} \Sigma_{U U}^{\circ} \theta^{\circ}}\left(\begin{array}{c}
\sigma_{D M}^{\circ} \\
\Sigma_{U U}^{\circ} \theta^{\circ}
\end{array}\right)\left\{\left(\omega^{\circ}, \theta^{\circ \prime}\right) \hat{\Sigma}_{\mathcal{R} W}-\left(1, \theta^{\circ \prime}\right) \hat{\Sigma}_{\Delta W}\right\} .
\end{aligned}
$$

Noting that, see (B.27),

$$
\hat{\Sigma}_{\mathcal{R} W}=\left(\begin{array}{c}
(1,0) \hat{\Sigma}_{\varepsilon W} \\
\hat{\Sigma}_{U W}
\end{array}\right), \quad \hat{\Sigma}_{\Delta W}=\left(\begin{array}{c}
(0,1) \hat{\Sigma}_{\varepsilon W} \\
\hat{\Sigma}_{U W}
\end{array}\right)
$$

it is seen that $\left(\omega^{\circ}, \theta^{\circ \prime}\right) \hat{\Sigma}_{\mathcal{R} W}-\left(1, \theta^{\circ \prime}\right) \hat{\Sigma}_{\Delta W}=\left(\omega^{\circ},-1\right) \hat{\Sigma}_{\varepsilon W}$. The expression for $S_{\mathcal{R} \mathcal{R} \cdot \Delta}^{-1}$ in (B.28) implies

$$
\begin{aligned}
& S_{\mathcal{R} \mathcal{R} \cdot \Delta}^{\circ-1}\left(\begin{array}{c}
\sigma_{D M}^{\circ} \\
\Sigma_{U U}^{\circ} \theta^{\circ}
\end{array}\right) \\
\stackrel{a . s .}{=} & \left\{\left(\begin{array}{cc}
\sigma_{M M}^{\circ-1} & 0 \\
0 & \Sigma_{U U}^{\circ-1}
\end{array}\right)+\frac{1}{\sigma_{D D \cdot M}^{\circ}}\left(\begin{array}{c}
\omega^{\circ} \\
\theta^{\circ}
\end{array}\right)^{\otimes 2}\right\}\left(\begin{array}{c}
\sigma_{D M}^{\circ} \\
\Sigma_{U U}^{\circ} \theta^{\circ}
\end{array}\right)+\mathrm{o}\left(T^{\eta-1 / 2}\right) \\
= & \left(\begin{array}{c}
\omega^{\circ} \\
\theta^{\circ}
\end{array}\right) \frac{\sigma_{D D}^{\circ}+\theta^{\circ \prime} \Sigma_{U U}^{\circ} \theta^{\circ}}{\sigma_{D D \cdot M}^{\circ}}+\mathrm{o}\left(T^{\eta-1 / 2}\right),
\end{aligned}
$$


where $\left(\omega^{\circ}, \theta^{\circ \prime}\right) \mathcal{H}^{\circ}=\left(\omega^{\circ},-1\right) \tau_{\perp}^{\circ}$ by (B.10). Inserting these results in (B.31) shows

$$
\mathcal{H}^{\circ \prime}\left(S_{\mathcal{R} \mathcal{R} \cdot \Delta}^{\circ-1} \hat{\Sigma}_{\mathcal{R} W \cdot \Delta}-S_{\mathcal{R} \mathcal{R}}^{\circ-1} \hat{\Sigma}_{\mathcal{R} W}\right) \stackrel{a . s .}{=} \tau_{\perp}^{\circ \prime}\left(\begin{array}{c}
\omega^{\circ} \\
-1
\end{array}\right)^{\otimes 2} \sigma_{D D \cdot M}^{\circ-1} \hat{\Sigma}_{\varepsilon W}\left\{1+\mathrm{o}\left(T^{\eta-1 / 2}\right)\right\} .
$$

Further, note that $S_{\varepsilon_{M} \varepsilon_{M}}^{\circ} \stackrel{\text { a.s. }}{=} \sigma_{M M}^{\circ}+T^{-1 / 4}$ and $\hat{\Sigma}_{\varepsilon_{M} W}=(1,0) \hat{\Sigma}_{\varepsilon W}$ along with the identies (B.29) to see

$$
\mathcal{G} S_{M M}^{-1} \hat{\Sigma}_{\varepsilon_{M} W} \stackrel{a . s .}{=} \sigma_{M M}^{-1} \tau_{\perp}^{\circ \prime}\left(\begin{array}{l}
1 \\
0
\end{array}\right)^{\otimes 2} \hat{\Sigma}_{\varepsilon W}\left\{1+\mathrm{o}\left(T^{\eta-1 / 2}\right)\right\} .
$$

Combining the two last expressions and noting $\hat{\Sigma}_{\varepsilon W}=\mathrm{o}\left(T^{1 / 4-\eta}\right)$ for some $\eta>0$ shows

$$
\begin{aligned}
& \mathcal{G} S_{M M}^{-1} \hat{\Sigma}_{\varepsilon_{M} W}+\mathcal{H}^{\prime}\left(S_{\mathcal{R} \mathcal{R} \cdot \Delta}^{\circ-1} \hat{\Sigma}_{\mathcal{R} W \cdot \Delta}-S_{\mathcal{R} \mathcal{R}}^{\circ-1} \hat{\Sigma}_{\mathcal{R} W}\right) \\
& \stackrel{\text { a.s. }}{=} \tau_{\perp}^{\circ}\left\{\sigma_{M M}^{-1}\left(\begin{array}{c}
1 \\
0
\end{array}\right)^{\otimes 2}+\sigma_{D D \cdot M}^{-1}\left(\begin{array}{c}
\omega^{\circ} \\
-1
\end{array}\right)^{\otimes 2}\right\} \hat{\Sigma}_{\varepsilon W}+\mathrm{o}\left(T^{-1 / 4}\right) .
\end{aligned}
$$

Finally, the desired result follows by the partitioned inversion formula (B.30).

\section{B.5 Improving the rate of consistency}

Lemma B.9 Consider the maximum likelihood estimators in model $\mathrm{M}_{1 D S B}$. Assume $A, B, C$. Then $\hat{R}-R=\mathrm{o}\left(T^{1 / 4} \rho^{-T}\right)$ a.s.

Proof of Lemma B.9. Lemma B.4 shows that $\hat{R}-R_{\circ}=\mathrm{o}\left(T^{1 / 2} \rho^{-T}\right)$. Thus it suffices to analyse the profile likelihood $\ell(R)$ in a neighbourhood of $R_{\circ}$. Lemma B.6 shows that the profile likelihood $\ell(R)$ is maximised by maximising $\tilde{\ell}(R)$ up to an error of order o(1) uniformly over intervals $\left|R-R_{\circ}\right| \leq c T^{1 / 2} \rho_{\circ}^{-T}$ for any $c>0$. Thus, consider the approximate score equation

$$
0=\tilde{\ell}^{\prime}(R)=\tilde{\ell}^{\prime}\left(R_{\circ}\right)+\tilde{\ell}^{\prime \prime}\left(R_{\circ}\right)\left(R-R_{\circ}\right)+\frac{1}{2} \tilde{\ell}^{\prime \prime \prime}\left(R_{\circ}\right)\left(R_{*}-R_{\circ}\right)^{2}
$$

for some $R_{*}$ so $\left|R_{*}-R_{\circ}\right| \leq\left|R-R_{\circ}\right|$. Thus it holds

$$
R-R_{\circ}=\frac{\tilde{\ell}^{\prime}\left(R_{\circ}\right)+2^{-1} \tilde{\ell}^{\prime \prime \prime}\left(R_{\circ}\right)\left(R_{*}-R_{\circ}\right)^{2}}{-\tilde{\ell}^{\prime \prime}\left(R_{\circ}\right)} .
$$

Insert the results of Lemma B.7 to get the desired result. 
Lemma B.10 Consider the maximum likelihood estimators in model $\mathrm{M}_{1 D S B}$. Assume $A, B, C$. Then

(i) $\hat{R}-R_{\circ} \stackrel{\text { a.s. }}{=}\left\{-\tilde{\ell}^{\prime \prime}\left(R_{\circ}\right)\right\}^{-1} \tilde{\ell}^{\prime}\left(R_{\circ}\right)+\mathrm{o}\left(T^{-1 / 4} \rho_{\circ}^{-T}\right)$.

(ii) $\rho_{\circ}^{T}\left(\hat{R}-R_{\circ}\right) \tau_{\perp}^{\circ} \stackrel{\text { a.s. }}{=} \mathcal{P}_{\tau_{\perp}}^{\circ} \hat{\Sigma}_{\varepsilon W} \Sigma_{W W}^{o-1}\{1+\mathrm{o}(1)\}+\mathrm{o}(1)$.

Proof of Lemma B.10. (i) Lemma B.9 shows that $\hat{R}-R_{\circ}=\mathrm{o}\left(T^{1 / 4} \rho^{-T}\right)$. Insert this and the results of Lemma B.7 into (B.32) to get

$$
\hat{R}-R_{\circ}=\left\{-\tilde{\ell}^{\prime \prime}\left(R_{\circ}\right)\right\}^{-1} \tilde{\ell}^{\prime}\left(R_{\circ}\right)+\left\{-2 \tilde{\ell}^{\prime \prime}\left(R_{\circ}\right)\right\} \tilde{\ell}^{\prime \prime \prime}\left(R_{\circ}\right)\left(R_{*}-R_{\circ}\right)^{2}
$$

where $\left|R_{*}-R_{\circ}\right| \leq\left|\hat{R}-R_{\circ}\right|$. The second term is $\mathrm{o}\left(T^{-3 / 4} \rho_{\circ}^{3 T}\right)\left(T^{1 / 4} \rho_{\circ}^{-T}\right)^{2} \rho_{\circ}^{-2 T}=$ $\mathrm{o}\left(T^{-1 / 4} \rho_{\circ}^{-T}\right)$ so $(i)$ follows.

(ii) Insert the expressions in Lemma B.8 into (i) so that

$$
\hat{R}-R_{\circ} \stackrel{a . s .}{=} \frac{\rho_{\circ}^{T}\left\{\tau_{\perp}^{\circ} \Omega_{\circ}^{-1} \hat{\Sigma}_{\varepsilon W}+\mathrm{o}\left(T^{-1 / 4}\right)\right\}}{\rho_{\circ}^{2 T} \tau_{\perp}^{\circ \prime} \Omega_{\circ}^{-1} \tau_{\perp}^{\circ} \Sigma_{W W}^{\circ}}\{1+\mathrm{o}(1)\}+\mathrm{o}\left(\rho_{\circ}^{-T}\right) .
$$

Rearrange to get the desired result.

\section{B.6 Asymptotic distribution of estimators}

Theorem B.11 Consider the maximum likelihood estimators in model $\mathrm{M}_{1 D S B}$. Assume $A, B, C$. Then

(i) $\left\{\left(\sum_{t=1}^{T} M_{t}^{2}\right)^{1 / 2}\left(\hat{\omega}-\omega^{\circ}\right),\left(\sum_{t=1}^{T} U_{t}^{2}\right)^{1 / 2}\left(\hat{\theta}-\theta^{\circ}\right)\right\} \stackrel{\mathrm{D}}{\rightarrow} \mathrm{N}\left(0, \sigma_{D D \cdot M}^{2} I_{2 k-1}\right)$.

(ii) $\hat{\sigma}_{M M} \rightarrow \sigma_{M M}, \hat{\sigma}_{D D \cdot M} \rightarrow \sigma_{D D \cdot M}$ a.s.

(iii) Let $H=\left(\tau_{\perp}^{\circ} \Omega_{\circ}^{-1} \tau_{\perp}^{\circ}\right)^{-1 / 2} \tau_{\perp}^{\circ \prime} \Omega_{\circ}^{-1}\left\{\sum_{t=1}^{T} \rho^{2(t-T)}\right\}^{-1 / 2} \sum_{t=1}^{T} \rho^{t-T} \varepsilon_{t}^{\circ}$. Then it holds $\left\{-\ell^{\prime \prime}(\hat{R})\right\}^{1 / 2}\left(\hat{R}-R_{\circ}\right)=H+\mathrm{o}(1)$ a.s.

(iv) If $\left(\tau_{\perp}^{\circ} \Omega_{\circ}^{-1} \tau_{\perp}^{\circ}\right)^{-1 / 2} \tau_{\perp}^{\circ} \Omega_{\circ}^{-1} \varepsilon_{t}^{\circ}$ are independent $\mathrm{N}(0,1)$ then $H$ is $\mathrm{N}(0,1)$.

Proof of Theorem B.11. (i) Since $\hat{\rho}-\rho_{\circ}=o\left(T^{1 / 4} \rho_{\circ}^{-T}\right)$ by Lemma B.9 and since $\Delta_{1} D_{t}=\left(\omega^{\circ}, \theta^{\circ \prime}\right) \mathcal{R}_{t}^{\circ}+\varepsilon_{D \cdot M, t}^{\circ}$ then Lemma B.2 implies that

$$
\begin{aligned}
\left(\hat{\omega}-\omega^{\circ}, \hat{\theta}^{\prime}-\theta^{\circ}\right)\left(\sum_{t=1}^{T} \mathcal{R}_{t}^{\otimes 2}\right)^{1 / 2} & =\sum_{t=1}^{T} \varepsilon_{D \cdot M, t}^{\circ} \mathcal{R}_{t}^{\prime}\left(\sum_{t=1}^{T} \mathcal{R}_{t}^{\otimes 2}\right)^{-1 / 2} \\
& \stackrel{\text { a.s. }}{=} \sum_{t=1}^{T} \varepsilon_{D \cdot M, t}^{\circ} \mathcal{R}_{t}^{\circ \prime}\left(\sum_{t=1}^{T} \mathcal{R}_{t}^{\circ \otimes 2}\right)^{-1 / 2}+\mathrm{o}(1) .
\end{aligned}
$$

which is asymptotic normal. Similarly $\sum_{t=1}^{T} \mathcal{R}_{t}^{\otimes 2}=\sum_{t=1}^{T} \mathcal{R}_{t}^{\circ \otimes 2}\{1+\mathrm{o}(1)\}$ where $\mathcal{R}_{t}^{\circ}$ has asymptotically uncorrelated components $\varepsilon_{M, t}^{\circ}, U_{t-1}^{\circ}$.

(ii) First, consider $\hat{\sigma}_{M M}=T^{-1} \sum_{t=1}^{T} M_{t}^{2}$. Since $\hat{\rho}-\rho_{\circ}=\mathrm{o}\left(T^{1 / 4} \rho_{\circ}^{-T}\right)$ by Lemma B.9 then Lemma B.2 implies $\hat{\sigma}_{M M}=T^{-1} \sum_{t=1}^{T} M_{t}^{\circ 2}+\mathrm{o}(1)$ which has the desired limit. 
Secondly, consider $\hat{\sigma}_{D D \cdot M}=T^{-1} \sum_{t=1}^{T}\left(\Delta_{1} D_{t} \mid \mathcal{R}_{t}\right)$. Noting $\Delta_{1} D_{t}=\left(\omega^{\circ}, \theta^{\circ \prime}\right) \mathcal{R}_{t}^{\circ}+$ $\varepsilon_{D \cdot M, t}^{\circ}$ then in the same way Lemma B.2 implies $\hat{\sigma}_{D D \cdot M}=T^{-1} \sum_{t=1}^{T} \varepsilon_{D \cdot M, t}^{\circ 2}+\mathrm{o}(1)$ which has the desired limit.

(iii) Combine Lemmas B.6, B.8, B.10(ii) to see that

$$
\left\{-\ell^{\prime \prime}(\hat{R})\right\}^{1 / 2}\left(\hat{R}-R_{\circ}\right) \stackrel{a . s .}{=}\left(\tau_{\perp}^{\circ} \Omega_{\circ}^{-1} \tau_{\perp}^{\circ}\right)^{-1 / 2} \tau_{\perp}^{\circ} \Omega_{\circ}^{-1} \hat{\Sigma}_{\varepsilon W} \Sigma_{W W}^{\circ-1 / 2}+\mathrm{o}(1) .
$$

then $\rho_{\circ}^{T}\left(\hat{R}-R_{\circ}\right)=\left(\tau_{\perp}^{\circ} \Omega_{\circ}^{-1} \tau_{\perp}^{\circ}\right)^{-1} \tau_{\perp}^{\circ} \Omega_{\circ}^{-1} \hat{\Sigma}_{\varepsilon W} \Sigma_{W W}^{\circ-1}\{1+\mathrm{o}(1)\}+\mathrm{o}(1)$ a.s. By an argument as in Anderson (1959), see also Nielsen (2010, Theorem 4) then $H=\hat{\Sigma}_{\varepsilon W} \Sigma_{W W}^{\circ-1 / 2}=$ $\left\{\sum_{t=1}^{T} \rho^{2(t-T)}\right\}^{-1 / 2} \sum_{t=1}^{T} \rho^{t-T} \varepsilon_{t}^{\circ}+\mathrm{o}(1)$ giving the desired result.

(iv) Under the normality assumption then $H$ is a linear combination of normals, so normal itself.

\section{B.7 Likelihood in restricted model}

Lemma B.12 Consider the maximum likelihood estimators in model $\mathrm{M}_{1 D S B}$. Assume $A, B, C$. Then

$$
\begin{aligned}
2 \ell(\hat{R}) \stackrel{\text { a.s. }}{=}-T \log \operatorname{det}\left(S_{\varepsilon \varepsilon}^{\circ}\right)+\sigma_{D D \cdot M}^{-1} \hat{\Sigma}_{\varepsilon_{D \cdot M} U} \Sigma_{U U}^{\circ-1} \hat{\Sigma}_{U \varepsilon_{D} \cdot M} & \\
& +\operatorname{tr}\left(\Omega_{\circ}^{-1} \mathcal{P}_{\tau_{\perp}}^{\circ} \hat{\Sigma}_{\varepsilon W} \Sigma_{W W}^{\circ-1} \hat{\Sigma}_{W \varepsilon}\right)+o(1) .
\end{aligned}
$$

Proof of Lemma B.12. The profile log likelihood is given in Lemma B.6 as

$$
2\left\{\ell(\hat{R})-\ell\left(R_{\circ}\right)\right\}=2\left\{\tilde{\ell}(\hat{R})-\tilde{\ell}\left(R_{\circ}\right)\right\}+\mathrm{o}(1) .
$$

Expanding $\tilde{\ell}(\hat{R})$ around $R_{\circ}$ then gives

$$
2\left\{\tilde{\ell}(\hat{R})-\tilde{\ell}\left(R_{\circ}\right)\right\}=2 \tilde{\ell}^{\prime}\left(R_{\circ}\right)\left(\hat{R}-R_{\circ}\right)+\tilde{\ell}^{\prime \prime}\left(R_{\circ}\right)\left(\hat{R}-R_{\circ}\right)^{2}+\frac{1}{3} \tilde{\ell}^{\prime \prime \prime}\left(R_{\circ}\right)\left(R_{*}-R_{\circ}\right)^{3} .
$$

where $\left|R_{*}-R_{\circ}\right| \leq\left|\hat{R}-R_{\circ}\right|$. Insert the expression for $\hat{R}-R_{\circ}$ from Lemma B.10(i) and use the bound $\hat{R}-R_{\circ}=\mathrm{o}\left(T^{1 / 4} \rho_{\circ}^{-T}\right)$ from Lemma B.4 to get

$$
\begin{aligned}
& 2\left\{\tilde{\ell}(\hat{R})-\tilde{\ell}\left(R_{\circ}\right)\right\} \stackrel{a . s .}{=}-\left\{\tilde{\ell}^{\prime \prime}\left(R_{\circ}\right)\right\}^{-1}\left\{\tilde{\ell}^{\prime}\left(R_{\circ}\right)\right\}^{2} \\
& +\mathrm{o}\left\{T^{-1 / 4} \rho_{\circ}^{-T} \tilde{\ell}^{\prime}\left(R_{\circ}\right)+\rho_{\circ}^{-2 T} \tilde{\ell}^{\prime \prime}\left(R_{\circ}\right)+T^{3 / 4} \rho_{\circ}^{-3 T} \tilde{\ell}^{\prime \prime \prime}\left(R_{\circ}\right)\right\} .
\end{aligned}
$$

Insert the bounds and the expressions for the derivatives established in Lemmas B.7, B.8 to see

$$
2\left\{\tilde{\ell}(\hat{R})-\tilde{\ell}\left(R_{\circ}\right)\right\} \stackrel{\text { a.s. }}{=}-\frac{\rho_{\circ}^{2 T}\left\{\tau_{\perp}^{\circ} \Omega_{\circ}^{-1} \hat{\Sigma}_{\varepsilon W}+\mathrm{o}\left(T^{-1 / 4}\right)\right\}^{2}}{\rho_{\circ}^{2 T} \tau_{\perp}^{\circ} \Omega_{\circ}^{-1} \tau_{\perp}^{\circ} \Sigma_{W W}^{\circ}}+\mathrm{o}(1) .
$$


Noting that $\hat{\Sigma}_{\varepsilon W}=\mathrm{o}\left(T^{1 / 4}\right)$ this reduces to

$$
2\left\{\tilde{\ell}(\hat{R})-\tilde{\ell}\left(R_{\circ}\right)\right\} \stackrel{\text { a.s. }}{=}-\left(\tau_{\perp}^{\circ} \Omega_{\circ}^{-1} \tau_{\perp}^{\circ}\right)^{-1} \tau_{\perp}^{\circ} \Omega_{\circ}^{-1} \hat{\Sigma}_{\varepsilon W} \Sigma_{W W}^{\circ-1} \Sigma_{W \varepsilon}^{\circ} \Omega_{\circ}^{-1} \tau_{\perp}^{\circ}+\mathrm{o}(1) .
$$

Taking trace and rearranging shows

$$
2\left\{\tilde{\ell}(\hat{R})-\tilde{\ell}\left(R_{\circ}\right)\right\} \stackrel{\text { a.s. }}{=} \operatorname{tr}\left(\Omega_{\circ}^{-1} \mathcal{P}_{\tau_{\perp}}^{\circ} \hat{\Sigma}_{\varepsilon W} \Sigma_{W W}^{\circ-1} \Sigma_{W \varepsilon}^{\circ}\right)+\mathrm{o}(1)
$$

Insert this in (B.33) and use the expression for $2 \ell\left(R_{\circ}\right)$ in Lemma B.3.

\section{B.8 Likelihood in unrestricted model}

The unrestricted model $M_{1 D}$ is now analysed. An expression for the likelihood value was given in Nielsen (2010) but this is not directly applicable here and needs to be elaborated. Lemma A.12 of the same paper gives an improved consistency rate for some of the parameters of $\mathrm{M}_{1 D}$. That result is now extended for all parameters. For results in the unrestricted model the data generating process is

$$
\Delta_{1} \Delta_{\rho_{\circ}} X_{t}=\alpha_{1}^{\circ} \beta_{1}^{* \circ \prime} \Delta_{\rho_{\circ}} X_{t-1}^{*}+\alpha_{\rho}^{\circ} \beta_{\rho}^{\circ \prime} \Delta_{1} X_{t-1}+\sum_{j=1}^{k-2} \Phi_{j}^{\circ} \Delta_{1} \Delta_{\rho_{\circ}} X_{t-j}+\varepsilon_{t}^{\circ} .
$$

where $\beta_{1}^{*}=\left(\beta_{1}^{\prime}, \delta^{\prime}\right)^{\prime}$ and $\Delta_{\rho_{\circ}} X_{t-1}^{*}=\left(\Delta_{\rho_{\mathrm{o}}} X_{t-1}, 1\right)$. This of course encompasses the data generating process (B.7) under $\mathrm{M}_{1 D S B}$.

Lemma B.13 Suppose $\mathrm{M}_{1 D}$ holds with $\rho_{\circ} \geq \varrho$ for some $\varrho>1$. Assume $A, B, C$. Recall the definitions of $\tau_{\perp}^{\circ}, \mathcal{P}_{\tau_{\perp}}^{\circ}, \mathcal{P}_{\alpha}^{\circ}$ in $(B .8),(B .9)$. Then

(i) $\hat{\boldsymbol{\alpha}}-\boldsymbol{\alpha}=T^{-1 / 2} \hat{\Sigma}_{\varepsilon U} \Sigma_{U U}^{-1}+\mathrm{OP}_{\mathrm{P}}\left(T^{-\xi}\right)=\mathrm{O}_{\mathrm{P}}\left(T^{-1 / 2}\right)$.

(ii) $\alpha_{1}^{\circ}\left(\hat{\beta}_{1}^{* D}-\beta_{1}^{* \circ}\right)^{\prime} \overline{\hat{\beta}}_{1 \perp}^{* D} N_{V}^{-1}=T^{-1 / 2} \mathcal{P}_{\alpha}^{\circ} \hat{\Sigma}_{\varepsilon V} \hat{\Sigma}_{V V}^{-1}+\mathrm{O}_{\mathrm{P}}\left(T^{-1 / 2}\right)=\mathrm{O}_{\mathrm{P}}\left(T^{-1 / 2}\right)$.

(iii) $\rho_{\circ}^{-T} \breve{\tau}^{D \prime} \sum_{t=1}^{T} \hat{\varepsilon}_{t}^{D} W_{t-1}^{\circ}=\rho_{\circ}^{-T} \tau^{\circ \prime} \sum_{t=1}^{T} \varepsilon_{t}^{\circ} W_{t-1}^{\circ}+\mathrm{OP}_{\mathrm{P}}(1)$.

(iv) $\hat{\Omega}_{D}-\Omega_{\circ}=T^{-1} \sum_{t=1}^{T}\left\{\left(\varepsilon_{t}^{\circ}\right)^{\otimes 2}-\Omega_{\circ}\right\}+\mathrm{OP}\left(T^{-1 / 2}\right)=\mathrm{O}_{\mathrm{P}}\left(T^{-1 / 2}\right)$.

(v) $\tau_{\perp}^{\circ}\left(\hat{\rho}_{H}-\rho_{\circ}\right)=\mathcal{P}_{\tau_{\perp}} \sum_{t=1}^{T} \varepsilon_{t}^{\circ} W_{t-1}^{\circ}\left\{\sum_{t=1}^{T}\left(W_{t-1}^{\circ}\right)^{2}\right\}^{-1}\left\{1+\mathrm{O}_{\mathrm{P}}\left(T^{-1 / 2}\right)\right\}+\mathrm{OP}\left(T^{-1 / 4} \rho_{\circ}^{-T}\right)$.

Proof of Lemma B.13. Product moments. Let $U_{t-1}^{D}, V_{t-1}^{D}$ denote $U_{t-1}, V_{t-1}$ computed at $\hat{\rho}_{D}$. Combine (A.12) and the first display on p.911 of Nielsen (2010) to get

$$
\begin{aligned}
& \left(\begin{array}{cccc}
\hat{S}_{U U} & \hat{S}_{U V} & \hat{S}_{U \varepsilon} & \hat{S}_{U W} \\
* & \hat{S}_{V V} & \hat{S}_{V \varepsilon} & \hat{S}_{V W} \\
* & * & \hat{S}_{\varepsilon \varepsilon} & \hat{S}_{\varepsilon W} \\
* & * & * & \hat{S}_{W W}
\end{array}\right)=\frac{1}{T} \sum_{t=1}^{T}\left(\begin{array}{c}
U_{t-1}^{D} \\
N_{V} V_{t-1}^{D} \\
\varepsilon_{t}^{\circ} \\
N_{W} W_{t-1}^{\circ}
\end{array}\right)^{\otimes 2} \\
& =\left(\begin{array}{cccc}
\Sigma_{U U}+\mathrm{OP}(1) & \mathrm{OP}\left(T^{-\xi / 2}\right) & T^{-1 / 2}\left\{\hat{\Sigma}_{U \varepsilon}+\mathrm{OP}(1)\right\} & \mathrm{OP}\left(T^{-\xi / 2}\right) \\
* & \hat{\Sigma}_{V V}+\mathrm{OP}(1) & T^{-1 / 2}\left\{\hat{\Sigma}_{V \varepsilon}+\mathrm{OP}_{\mathrm{P}}(1)\right\} & \mathrm{OP}\left(T^{-\xi / 2}\right) \\
* & * & \Omega_{\circ}+\mathrm{OP}_{\mathrm{P}}(1) & T^{-1 / 2} \hat{\Sigma}_{\varepsilon W} \\
* & * & * & \Sigma_{W W}+\mathrm{OP}_{\mathrm{P}}(1)
\end{array}\right)
\end{aligned}
$$


where $\hat{\Sigma}_{W \varepsilon}=\rho_{\circ}^{-T} \sum_{t=1}^{T} W_{t-1}^{\circ} \varepsilon_{t}^{\circ}, \hat{\Sigma}_{U \varepsilon}=T^{-1 / 2} \sum_{t=1}^{T} U_{t-1}^{\circ} \varepsilon_{t}^{\circ}, \hat{\Sigma}_{V \varepsilon}=T^{-1 / 2} N_{V} \sum_{t=1}^{T} V_{t-1}^{\circ} \varepsilon_{t}^{\circ}$, and $\hat{\Sigma}_{V V}=T^{-1} \sum_{t=1}^{T}\left(N_{V} V_{t-1}^{\circ}\right)^{\otimes 2}$.

(i) The estimator for $\hat{\boldsymbol{\alpha}}$ is then

$$
\hat{\boldsymbol{\alpha}}=\sum_{t=1}^{T}\left(\Delta_{1} \Delta_{\hat{\rho}} X_{t}\right)\left(U_{t-1}^{D}\right)^{\prime}\left\{\sum_{t=1}^{T}\left(U_{t-1}^{D}\right)^{\otimes 2}\right\}^{-1} .
$$

An equation shown in the proof of Lemma A.7 of Nielsen (2010) gives

$$
\Delta_{1} \Delta_{\hat{\rho}} X_{t}=\varepsilon_{t}^{\circ}+\left(\boldsymbol{\alpha}+\hat{\delta}_{U}\right) U_{t-1}^{D}+\hat{\delta}_{V} N_{V} V_{t-1}^{D}+\hat{\delta}_{W} W_{t-1}^{\circ},
$$

where $\hat{\delta}_{U}=\left(\hat{\rho}_{D}-\rho_{\circ}\right)\left\{\alpha_{1}^{\circ} /\left(1-\hat{\rho}_{D}\right),-\breve{\Psi}_{\hat{\rho}}^{D} \bar{\beta}_{1}, \hat{\rho}_{D}^{0} \sum_{j=1}^{k-2} \Phi_{j}^{\circ}, \ldots, \hat{\rho}_{D}^{k-3} \sum_{j=k-2}^{k-2} \Phi_{j}^{\circ}\right\}$ and $\hat{\delta}_{V}=$ $\alpha_{1}^{\circ} \beta_{1}^{* \circ} \overline{\hat{\beta}}_{1 \perp}^{* D} N_{V}^{-1}\left(1-\rho_{\circ}\right) /\left(1-\hat{\rho}_{D}\right)$ and $\hat{\delta}_{W}=-\left(\hat{\rho}_{D}-\rho_{\circ}\right) \breve{\tau}_{\perp}^{D}$. It follows that

$$
\begin{aligned}
\hat{\boldsymbol{\alpha}}-\boldsymbol{\alpha}= & \sum_{t=1}^{T} \varepsilon_{t}^{\circ}\left(U_{t-1}^{D}\right)^{\prime}\left\{\sum_{t=1}^{T}\left(U_{t-1}^{D}\right)^{\otimes 2}\right\}^{-1} \\
& +\hat{\delta}_{U}+\sum_{t=1}^{T}\left(\hat{\delta}_{V} N_{V} V_{t-1}^{D}+\hat{\delta}_{W} W_{t-1}^{\circ}\right)\left(U_{t-1}^{D}\right)^{\prime}\left\{\sum_{t=1}^{T}\left(U_{t-1}^{D}\right)^{\otimes 2}\right\}^{-1} .
\end{aligned}
$$

Lemma A.11 of Nielsen (2010) shows that $T^{1 / 2} \rho_{\circ}^{T}\left(\hat{\rho}_{D}-\rho_{\circ}\right)$ and $\beta_{1}^{* o \prime} \overline{\hat{\beta}}_{1 \perp}^{* D} N_{V}^{-1}$ are $\mathrm{O}_{\mathrm{P}}\left(T^{-\xi / 2}\right)$. This implies that $\hat{\delta}_{U}, \hat{\delta}_{W}=\mathrm{OP}\left(\rho_{\circ}^{-T} T^{(1-\xi) / 2}\right)$ and $\hat{\delta}_{V}=\mathrm{O}_{\mathrm{P}}\left(T^{-\xi / 2}\right)$. From (B.34) it follows that

$$
\begin{aligned}
\hat{\boldsymbol{\alpha}}-\boldsymbol{\alpha}= & \left\{T^{-1 / 2} \Sigma_{\varepsilon U}+\mathrm{op}_{\mathrm{P}}\left(T^{-1 / 2}\right)+\mathrm{OP}\left(T^{-\xi}\right)\right\}\left\{\Sigma_{U U}^{-1}+\mathrm{op}_{\mathrm{P}}\left(T^{-\xi / 2}\right)\right\} \\
& +\mathrm{O}_{\mathrm{P}}\left(\rho_{\circ}^{-T} T^{(1-\xi) / 2}\right)+\mathrm{oP}_{\mathrm{P}}\left(T^{-\xi / 2}\right) \mathrm{OP}_{\mathrm{P}}\left(T^{-\xi / 2}\right)+\mathrm{op}_{\mathrm{P}}\left(\rho_{\circ}^{-T} T^{(1-\xi) / 2}\right) \mathrm{O}_{\mathrm{P}}\left(T^{(-1-\xi) / 2} \rho_{\circ}^{T}\right) .
\end{aligned}
$$

By Assumption B then $\xi>1 / 2$ and the desired result follows.

(ii, iii) Statement of Lemma A.12(ii, iii) of Nielsen (2010).

(iv) The variance estimator is $\hat{\Omega}_{D}=T^{-1} \sum_{t=1}^{T}\left(\Delta_{1} \Delta_{\hat{\rho}} X_{t} \mid U_{t-1}^{D}\right)^{\otimes 2}$. Due to (B.35) then

$$
\hat{\Omega}_{D}=T^{-1} \sum_{t=1}^{T}\left(\varepsilon_{t}^{\circ}+\hat{\delta}_{V} N_{V} V_{t-1}^{D}+\hat{\delta}_{W} W_{t-1}^{\circ} \mid U_{t-1}^{D}\right)^{\otimes 2} .
$$

From (ii, iii) it follows that $\hat{\delta}_{V}=-T^{-1 / 2} \mathcal{P}_{\alpha}^{\circ} \hat{\Sigma}_{\varepsilon V} \hat{\Sigma}_{V V}^{-1}+\mathrm{O}_{\mathrm{P}}\left(T^{-\xi}\right)=\mathrm{O}_{\mathrm{P}}\left(T^{-1 / 2}\right)$. Inserting this and using (B.34) it follows that $\hat{\Omega}_{D}=S_{\varepsilon \varepsilon}^{\circ}+T^{-1} G$ where

$$
\begin{aligned}
G= & -\Sigma_{\varepsilon U} \Sigma_{U U}^{-1} \Sigma_{U \varepsilon}+\mathrm{OP}_{\mathrm{P}}(1)+\hat{\delta}_{W} \mathrm{OP}\left(\rho_{\circ}^{T} T^{-1-\xi / 2}\right) \\
& +\mathcal{P}_{\alpha}^{\circ} \hat{\Sigma}_{\varepsilon V} \hat{\Sigma}_{V V}^{-1} \hat{\Sigma}_{V \varepsilon} \mathcal{P}_{\alpha}^{\circ \prime}-\mathcal{P}_{\alpha}^{\circ} \hat{\Sigma}_{\varepsilon V} \hat{\Sigma}_{V V}^{-1} \hat{\Sigma}_{V \varepsilon}-\hat{\Sigma}_{\varepsilon V} \hat{\Sigma}_{V V}^{-1} \hat{\Sigma}_{V \varepsilon} \mathcal{P}_{\alpha}^{\circ \prime} \\
& +\hat{\delta}_{W} \hat{\Sigma}_{W W} \hat{\delta}_{W}^{\prime}\left\{1+\mathrm{OP}\left(T^{-1 / 2}\right)\right\}+\rho_{\circ}^{-T}\left(\hat{\delta}_{W} \hat{\Sigma}_{W \varepsilon}+\hat{\Sigma}_{\varepsilon W} \hat{\delta}_{W}^{\prime}\right) .
\end{aligned}
$$


Since $\hat{\delta}_{W}=\mathrm{O}_{\mathrm{P}}\left(\rho_{\circ}^{-T} T^{(1-\xi) / 2}\right), \hat{\Sigma}_{W W}=\mathrm{O}(1)$ and $S_{W \varepsilon}=\mathrm{op}_{\mathrm{P}}\left(T^{(1-\xi) / 2}\right)$ and $\xi>1 / 2$ then

$$
\begin{aligned}
G= & -\Sigma_{\varepsilon U} \Sigma_{U U}^{-1} \Sigma_{U \varepsilon}+\mathcal{P}_{\alpha}^{\circ} \hat{\Sigma}_{\varepsilon V} \hat{\Sigma}_{V V}^{-1} \hat{\Sigma}_{V \varepsilon} \mathcal{P}_{\alpha}^{\circ \prime}-\mathcal{P}_{\alpha}^{\circ} \hat{\Sigma}_{\varepsilon V} \hat{\Sigma}_{V V}^{-1} \hat{\Sigma}_{V \varepsilon}-\hat{\Sigma}_{\varepsilon V} \hat{\Sigma}_{V V}^{-1} \hat{\Sigma}_{V \varepsilon} \mathcal{P}_{\alpha}^{\circ \prime} \\
& +\hat{\delta}_{W} \hat{\Sigma}_{W W} \hat{\delta}_{W}^{\prime}+\rho_{\circ}^{-T}\left(\hat{\delta}_{W} \hat{\Sigma}_{W \varepsilon}+\hat{\Sigma}_{\varepsilon W} \hat{\delta}_{W}^{\prime}\right)+\mathrm{op}_{\mathrm{P}}(1),
\end{aligned}
$$

and $G=\mathrm{OP}\left(T^{1 / 2}\right)$. It follows that $\hat{\Omega}_{D}=S_{\varepsilon \varepsilon}^{\circ}+\mathrm{OP}\left(T^{-1 / 2}\right)$. Since $S_{\varepsilon \varepsilon}^{\circ}=\Omega_{\circ}+\mathrm{O}_{\mathrm{P}}\left(T^{-1 / 2}\right)$ the desired result follows.

(v) Lemma A.9 of Nielsen (2010) shows

$$
\begin{aligned}
\hat{\tau}_{\perp}^{D \prime} \hat{\Omega}_{D}^{-1} \rho_{\circ}^{T}\left(\hat{\rho}_{H}-\rho_{\circ}\right)\left(\breve{\tau}_{\perp}^{D} \hat{S}_{W W \cdot U}-\hat{\mathcal{P}}_{\alpha} \breve{\tau}_{\perp}^{D} \hat{S}_{W V \cdot U} \hat{S}_{V V \cdot U}^{-1} \hat{S}_{V W \cdot U}\right) \\
\quad=T^{1 / 2} \hat{\tau}_{\perp}^{D} \hat{\Omega}_{D}^{-1}\left(\hat{S}_{\varepsilon W \cdot U}-\hat{\mathcal{P}}_{\alpha} \hat{S}_{\varepsilon V \cdot U} \hat{S}_{V V \cdot U}^{-1} \hat{S}_{V W \cdot U}\right)
\end{aligned}
$$

where $\hat{\mathcal{P}}_{\alpha}=\breve{\alpha}_{1}\left(\hat{\alpha}_{1}^{D \prime} \hat{\Omega}_{D}^{-1} \breve{\alpha}_{1}\right)^{-1} \hat{\alpha}_{1}^{D \prime} \hat{\Omega}_{D}^{-1}$. Exploit the $T^{1 / 2}$-order of the $\rho, \alpha, \tau, \Omega$ estimators by $(i, i i i)$ as well as premultiplying the equation by $\tau_{\perp}^{\circ}\left(\tau_{\perp}^{\circ \prime} \Omega_{\circ}^{-1} \tau_{\perp}^{\circ}\right)^{-1}$ and postmultiplying by $\hat{S}_{W W}^{-1}$ to get

$$
\begin{aligned}
\tau_{\perp}^{\circ} \rho_{\circ}^{T}\left(\hat{\rho}_{H}-\rho_{\circ}\right) & =\left\{\rho_{\circ}^{T}\left(\hat{\rho}_{H}-\rho_{\circ}\right) \mathcal{P}_{\tau_{\perp}} \mathcal{P}_{\alpha}^{\circ} \tau_{\perp}^{\circ} \hat{S}_{W V \cdot U} \hat{S}_{V V \cdot U}^{-1} \hat{S}_{V W \cdot U} \hat{S}_{W W \cdot U}^{-1}\right. \\
& \left.+\mathcal{P}_{\tau_{\perp}} T^{1 / 2}\left(\hat{S}_{\varepsilon W \cdot U}-\mathcal{P}_{\alpha}^{\circ} \hat{S}_{\varepsilon V \cdot U} \hat{S}_{V V \cdot U}^{-1} \hat{S}_{V W \cdot U}\right) \hat{S}_{W W \cdot U}^{-1}\right\}\left\{1+\mathrm{O}_{\mathrm{P}}\left(T^{-1 / 2}\right)\right\} .
\end{aligned}
$$

Exploit the $T^{-1 / 4} \rho_{\circ}^{T}$ consistency of $\hat{\rho}_{H}$ as well as (B.35) to get the desired result.

An expansion is needed for the variance estimator $\hat{\Omega}_{D}$ in the unrestricted model $\mathrm{M}_{1 D}$. Theorem 3 of Nielsen (2010) shows that the estimator $\hat{\Omega}_{D}$, called $\hat{\Omega}_{\mathrm{H}}$ in that paper, is consistent.

Lemma B.14 Suppose $\mathrm{M}_{1 D}$ holds with $\rho_{\circ} \geq \varrho$ for some $\varrho>1$. Assume $A, B, C$. Then

$$
\begin{aligned}
2 \hat{\ell}_{1 D}= & -T \log \operatorname{det} S_{\varepsilon^{\circ} \varepsilon^{\circ}}+\operatorname{tr}\left(\Omega_{\circ}^{-1} \hat{\Sigma}_{\varepsilon U} \Sigma_{U U}^{-1} \hat{\Sigma}_{U \varepsilon}\right) \\
& +\operatorname{tr}\left(\Omega_{\circ}^{-1} \mathcal{P}_{\alpha}^{\circ} \hat{\Sigma}_{\varepsilon V} \Sigma_{V V}^{-1} \hat{\Sigma}_{V \varepsilon}\right)+\operatorname{tr}\left(\Omega_{\circ}^{-1} \mathcal{P}_{\tau_{\perp}}^{\circ} \hat{\Sigma}_{\varepsilon W} \hat{\Sigma}_{W W}^{-1} \hat{\Sigma}_{W \varepsilon}\right)+\mathrm{OP}_{\mathrm{P}}(1) .
\end{aligned}
$$

Proof of Lemma B.14. Combine (B.36) where $\hat{\delta}_{W}=-\left(\hat{\rho}_{D}-\rho_{\circ}\right) \breve{\tau}_{\perp}^{D}$ with Lemma B.13 $(v)$ to get $\hat{\Omega}_{D}=S_{\varepsilon^{\circ} \varepsilon^{\circ}}+T^{-1} G$ where

$$
\begin{aligned}
G= & -\hat{\Sigma}_{\varepsilon U} \Sigma_{U U}^{-1} \hat{\Sigma}_{U \varepsilon}+\mathcal{P}_{\alpha}^{\circ} \hat{\Sigma}_{\varepsilon V} \hat{\Sigma}_{V V}^{-1} \hat{\Sigma}_{V \varepsilon} \mathcal{P}_{\alpha}^{\circ \prime}-\mathcal{P}_{\alpha}^{\circ} \hat{\Sigma}_{\varepsilon V} \hat{\Sigma}_{V V}^{-1} \hat{\Sigma}_{V \varepsilon}-\hat{\Sigma}_{\varepsilon V} \hat{\Sigma}_{V V}^{-1} \hat{\Sigma}_{V \varepsilon} \mathcal{P}_{\alpha}^{\circ \prime} \\
& +\mathcal{P}_{\tau_{\perp}}^{\circ} \hat{\Sigma}_{\varepsilon W} \hat{\Sigma}_{W W}^{-1} \hat{\Sigma}_{W \varepsilon} \mathcal{P}_{\tau_{\perp}}^{\circ \prime}-\mathcal{P}_{\tau_{\perp}}^{\circ} \hat{\Sigma}_{\varepsilon W} \hat{\Sigma}_{W W}^{-1} \hat{\Sigma}_{W \varepsilon}-\hat{\Sigma}_{\varepsilon W} \hat{\Sigma}_{W W}^{-1} \hat{\Sigma}_{W \varepsilon} \mathcal{P}_{\tau_{\perp}}^{\circ \prime}+\mathrm{OP}_{\mathrm{P}}(1) .
\end{aligned}
$$

Use the log determinant expansion in Lemma B.5 to get

$$
2 \hat{\ell}_{1 D}=-T \log \operatorname{det} \hat{\Omega}_{D}=-T \log \operatorname{det} S_{\varepsilon^{\circ} \varepsilon^{\circ}}-\operatorname{tr}\left(\hat{\Omega}_{D}^{-1} G\right) .
$$

Since $\hat{\Omega}_{D}$ is consistent and $\mathcal{P}_{\alpha}^{\circ \prime} \Omega_{\circ}^{-1} \mathcal{P}_{\alpha}^{\circ}=\Omega_{\circ}^{-1} \mathcal{P}_{\alpha}^{\circ}$ and $\mathcal{P}_{\alpha}^{\circ \prime} \Omega_{\circ}^{-1} \mathcal{P}_{\alpha}^{\circ}=\Omega_{\circ}^{-1} \mathcal{P}_{\alpha}^{\circ}$ and using the symmetry of the trace then

$$
-\operatorname{tr}\left(\hat{\Omega}_{D}^{-1} G\right)=\operatorname{tr}\left\{\Omega_{\circ}^{-1}\left(\hat{\Sigma}_{\varepsilon U} \Sigma_{U U}^{-1} \hat{\Sigma}_{U \varepsilon}+\mathcal{P}_{\alpha}^{\circ} \hat{\Sigma}_{\varepsilon V} \Sigma_{V V}^{-1} \hat{\Sigma}_{V \varepsilon}+\mathcal{P}_{\tau_{\perp}}^{\circ} \hat{\Sigma}_{\varepsilon W} \hat{\Sigma}_{W W}^{-1} \hat{\Sigma}_{W \varepsilon}\right\}+\mathrm{OP}_{\mathrm{P}}(1) .\right.
$$

Insert this in the expression for $2 \hat{\ell}_{D}$ to get the desired result. 


\section{B.9 Proof of main theorem}

Proof of Theorem 4.1. It holds that

$$
\operatorname{LR}\left(\mathrm{H}_{B}, \mathrm{H}_{S} \mid \mathrm{M}_{1 D}\right)=2 \hat{\ell}_{1 D}-2 \hat{\ell}_{1 D S B}
$$

Inserting results from Lemmas B.12, B.14 gives

$$
\begin{aligned}
\operatorname{LR}= & \left\{-T \log \operatorname{det} S_{\varepsilon^{\circ} \varepsilon^{\circ}}+\operatorname{tr}\left(\Omega_{\circ}^{-1} \hat{\Sigma}_{\varepsilon U} \Sigma_{U U}^{-1} \hat{\Sigma}_{U \varepsilon}\right)+\operatorname{tr}\left(\Omega_{\circ}^{-1} \mathcal{P}_{\alpha}^{\circ} \hat{\Sigma}_{\varepsilon V} \Sigma_{V V}^{-1} \hat{\Sigma}_{V \varepsilon}\right)\right. \\
& \left.+\operatorname{tr}\left(\Omega_{\circ}^{-1} \mathcal{P}_{\tau_{\perp}}^{\circ} \hat{\Sigma}_{\varepsilon W} \hat{\Sigma}_{W W}^{-1} \hat{\Sigma}_{W \varepsilon}\right)\right\}-\left\{-T \log \operatorname{det}\left(S_{\varepsilon \varepsilon}^{\circ}\right)\right. \\
& \left.+\sigma_{D D \cdot M}^{-1} \hat{\Sigma}_{\varepsilon_{D \cdot M} U} \Sigma_{U U}^{\circ-1} \hat{\Sigma}_{U \varepsilon_{D \cdot M}}+\operatorname{tr}\left(\Omega_{\circ}^{-1} \mathcal{P}_{\tau_{\perp}}^{\circ} \hat{\Sigma}_{\varepsilon W} \Sigma_{W W}^{\circ-1} \hat{\Sigma}_{W \varepsilon}\right)\right\}+\mathrm{OP}_{\mathrm{P}}(1) .
\end{aligned}
$$

This reduces to

$$
\begin{aligned}
\mathrm{LR}=\operatorname{tr}\left(\Omega_{\circ}^{-1} \hat{\Sigma}_{\varepsilon U} \Sigma_{U U}^{-1} \hat{\Sigma}_{U \varepsilon}\right)-\sigma_{D D \cdot M}^{\circ-1} \hat{\Sigma}_{\varepsilon_{D \cdot M} U} \Sigma_{U U}^{-1} \hat{\Sigma}_{U \varepsilon_{D \cdot M}} & \\
& +\operatorname{tr}\left(\Omega_{\circ}^{-1} \mathcal{P}_{\alpha}^{\circ} \hat{\Sigma}_{\varepsilon V} \Sigma_{V V}^{-1} \hat{\Sigma}_{V \varepsilon}\right)+\mathrm{OP}_{\mathrm{P}}(1) .
\end{aligned}
$$

In the first term partitioned inversion of $\Omega_{0}^{-1}$ gives

$$
\operatorname{tr}\left(\Omega_{\circ}^{-1} \hat{\Sigma}_{\varepsilon U} \Sigma_{U U}^{-1} \hat{\Sigma}_{U \varepsilon}\right)=\sigma_{M M}^{\circ-1} \hat{\Sigma}_{\varepsilon_{M} U} \Sigma_{U U}^{-1} \hat{\Sigma}_{U \varepsilon_{M}}+\sigma_{D D \cdot M}^{\circ-1} \hat{\Sigma}_{\varepsilon_{D \cdot M} U} \Sigma_{U U}^{-1} \hat{\Sigma}_{U \varepsilon_{D \cdot M}}
$$

so the test statistic satisfies

$$
\mathrm{LR}=\sigma_{M M}^{\circ-1} \hat{\Sigma}_{\varepsilon_{M} U} \Sigma_{U U}^{-1} \hat{\Sigma}_{U \varepsilon_{M}}+\operatorname{tr}\left(\Omega_{\circ}^{-1} \mathcal{P}_{\alpha}^{\circ} \hat{\Sigma}_{\varepsilon V} \Sigma_{V V}^{-1} \hat{\Sigma}_{V \varepsilon}\right)+\mathrm{OP}(1) .
$$

Since $\varepsilon_{M, t}, U_{t-1}$ are mutually independent a martingal central limit theorem, see Brown and Eagleson (1971), gives that the first term is asymptotically $\chi^{2}$ with $\operatorname{dim} U=2 k-2$ degrees of freedom.

The term $\mathcal{P}_{\alpha}^{\circ} \hat{\Sigma}_{\varepsilon V}$ is the stochastic integral of $B_{1, t, T}=N_{V} V_{t-1}^{\circ}$ with respect to $c=$ $T^{-1 / 2} \sum_{s=1}^{t} \alpha_{1}^{\circ \prime} \Omega_{0}^{-1} \varepsilon_{s}$. The process $B_{1, t, T}$ is a function of $T^{-1 / 2} \sum_{s=1}^{t} \alpha_{1 \perp}^{\circ \prime} \varepsilon_{s}$. Thus, $B_{1, t, T}$ and $B_{1, t, T}$ converge to asymptocally independent processes, so by a mixed Gaussian argument, see Johansen $(1995, \S 13.1)$, the last term is $\chi^{2}$ with $\operatorname{dim}\left(\alpha_{1}^{\circ} \Omega_{\circ}^{-1} \varepsilon_{t}\right) \operatorname{dim}(V)=$ 2 degrees of freedom.

It is left to argue that the last term is asymptotically independent of the previous two. The last one is based on the processes $B_{1, t, T}, B_{1, t, T}$ which are asymptotically independent of $\hat{\Sigma}_{\varepsilon_{M} \varepsilon_{D \cdot M}}, \hat{\Sigma}_{\varepsilon_{M} U}$, see Chan and Wei (1988, Theorem 2.2). Since $\hat{\Sigma}_{\varepsilon V}, \hat{\Sigma}_{V V}$ are functionals of $B_{1, t, T}, B_{1, t, T}$ then $\hat{\Sigma}_{\varepsilon V}, \hat{\Sigma}_{V V}$ are asymptotically independent of $\hat{\Sigma}_{\varepsilon_{M} \varepsilon_{D \cdot M}}, \hat{\Sigma}_{\varepsilon_{M} U}$.

It then follows that LR is asymptotically $\chi^{2}$ with $(2 k-2)+2=2 k$ degrees of freedom. 